\title{
Geologie, Paläontologie und Geochronologie des Eem-Beckens Neumark-Nord 2 und Vergleich mit dem Becken Neumark-Nord 1 [Geiseltal, Sachsen-Anhalt]
}

\author{
Jaqueline Strahl, Matthias R. Krbetschek, Joachim Luckert, Björn Machalett, Stefan Meng, Eric A. Oches, Ivo Rappsilber,
} Stefan Wansa, Ludwig Zöller

Kurzfassung: $\quad$ Den Schwerpunkt dieser Arbeit bilden die Ergebnisse sedimentologischer, palynologischer, malakologischer und chronometrischer Untersuchungen an Sedimenten aus dem Zentralbereich des Paläoseebeckens Neumark-Nord 2. Die interdisziplinären Untersuchungen an dem 11 m mächtigen Hauptprofil A und benachbarten Profilen zeigen übereinstimmend, dass die limnische Sedimentation vom Ende des Saale-Komplexes über die Eem-Warmzeit bis in die Weichsel-Kaltzeit erfolgte. Das Profil lässt Seespiegelschwankungen mit einer generellen Tendenz der Verflachung und Verlandung sowie wechselnde Sedimentationsraten erkennen. Durch die palynologischen Untersuchungen sind außerdem mit Erosion und Sedimentumlagerungen verbundene Hiaten festgestellt worden. Eine von LAURAT et al. (2006) und MANiA et al. (2008, 2010) im Profil ausgewiesene zusätzliche Warmzeit, die durch eine Kaltphase von der Eem-Warmzeit separiert und zudem jünger als das Interglazial von NN1 sein soll, existiert nicht.

Vor allem die palynostratigraphische, aber auch die malakologische Koinzidenz der Becken NN1 und NN2 belegt die Gleichaltrigkeit der Ablagerungen. Somit ist in Neumark-Nord zwischen der Saale-Grundmoräne der Zeitz-Phase und den periglaziären Bildungen der Weichsel-Kaltzeit nur eine Warmzeit nachweisbar, das Eem. Dies wird durch neue geochronologische Daten eindeutig verifiziert. Daher können die zuletzt von MANIA et al. (2010) dokumentierten Lagerungsbeziehungen, nach denen die Beckenfolge von NN2 über der von NN1 liegen soll, nicht bestätigt werden.

In Neumark-Nord werden die klimatischen Besonderheiten des Mitteldeutschen Trockengebietes während der Eem-Warmzeit deutlich. Insofern bietet der Vergleich der Eem-Vorkommen von Neumark-Nord, Gröbern und Grabschütz ein Lehrbeispiel für die standortspezifische Variabilität benachbarter synchroner Warmzeitprofile.

[Geology, palaeontonlogy and geochronology of the Eemian palaeo lake basin of Neumark-Nord 2 and its comparison with basin Neumark-Nord 1 [Geiseltal, Sachsen-Anhalt]]

Abstract:

The main focus of this study is on the results of the sedimentological, palynological, malacological and chronometric investigations of sediments from the central region of the palaeo lake basin Neumark-North 2. These interdisciplinary examinations of the main profile A, with a cross-section of $11 \mathrm{~m}$, and of neighbouring profiles concur in indicating that the limnic sedimentation took place from the end of the Saalian complex during the Eemian Warm Stage and up to the Weichselian glacial period. The section reveals variations in the lake water level with a general tendency to lowering and filling up, as well as varying sedimentation rates. The palynological investigations have also shown hiatuses connected with erosion and sediment transport. An additional temperate period separated from the Eemian by a cold phase and also more recent than the interglacial of NN 1, as identified in the section by LAURAT et al. (2006) and MANIA et al. (2008, 2010), does not exist.

Especially the palynostratigraphic but also the malacological coincidence between the NN1 and the NN2 basins demonstrates the simultaneity of the deposits. Thus in Neumark-North, between the Saalian till of the Zeitz phase and the periglacial sediments of the Weichselian, there is evidence of only one warm period, the Eemian. This is clearly documented by the new geochronological data. It is therefore not possible to confirm the sedimentation relationships most recently described by MANIA et al. (2010) according to which the basin sequence of NN2 was supposed to lie on top of NN1.

In Neumark-North the particular climatic conditions of the Central German dry region during the Eemian Interglacial are clearly recognisable. In this sense, the comparison between the Eemian findings for Neumark-North, Gröbern and Grabschütz provide an instructive example of the variability between neighbouring synchronous warm period sections in relation to specific sites.

Keywords: $\quad$ upper pleistocene, quaternary stratigraphy, palynostratigraphy, quaternary molluscs, lake sediments, Eemian, luminescence dating, amino acid geochronology, quaternary geochronology, Geiseltal

Addresses of authors: J. Strahl, Landesamt für Bergbau, Geologie und Rohstoffe Brandenburg, Inselstr. 26, 03046 Cottbus. E-Mail: Jaqueline.Strahl@lbgr. brandenburg.de; M. R. Krbetschek, Sächsische Akademie der Wissenschaften, Forschungsstelle Geochronologie Quartär, Institut für Angewandte Physik / TU Freiberg, Leipziger-Str. 23, 09596 Freiberg. E-Mail: quatmi@mailserver.tu-freiberg.de; J. Luckert, Landeslabor Berlin-Brandenburg, Fachbereich Bodenlabor (Röntgenlabor), Stahnsdorfer Damm 77, 14532 Kleinmachnow. E-Mail: joachim.luckert@landeslabor-bbb.de; B. Machalett, Humboldt-Universität zu Berlin, Geographisches Institut, Abteilung Klimatologie, Unter den Linden 6, 10099 Berlin; present address: Leverhulme Trust Visiting Fellow, Institute of Geography and Earth Sciences, Luminescence Laboratory, Aberystwyth University, Aberystwyth, Wales, SY23 3DB, U.K. E-Mail: b.machalett@nakula.de; S. Meng, Institut für Geographie und Geologie, Ernst-Moritz-Arndt-Universität Greifswald, Friedrich-Ludwig-Jahn-Str. 17a, 17487 Greifswald. E-Mail: stefan.meng@uni-greifswald.de; E. A. Oches, Natural \& Applied Sciences Department, Bentley University, Jennison Hall 126, 175 Forest St., Waltham, MA 02452, USA. E-mail: roches@bentley.edu; I. Rappsilber, Landesamt für Geologie und Bergwesen Sachsen-Anhalt, Postfach 156, 06035 Halle. E-Mail: Rappsilber@lagb.mw.sachsen-anhalt.de; S. Wansa, Landesamt für Geologie und Bergwesen Sachsen-Anhalt, Postfach 156, 06035 Halle. E-Mail: Wansa@lagb.mw.sachsen-anhalt.de; L. Zöller, Lehrstuhl Geomorphologie, Universität Bayreuth, Universitätsstraße 30, 95440 Bayreuth. E-Mail: Ludwig.Zoeller@uni-bayreuth.de 


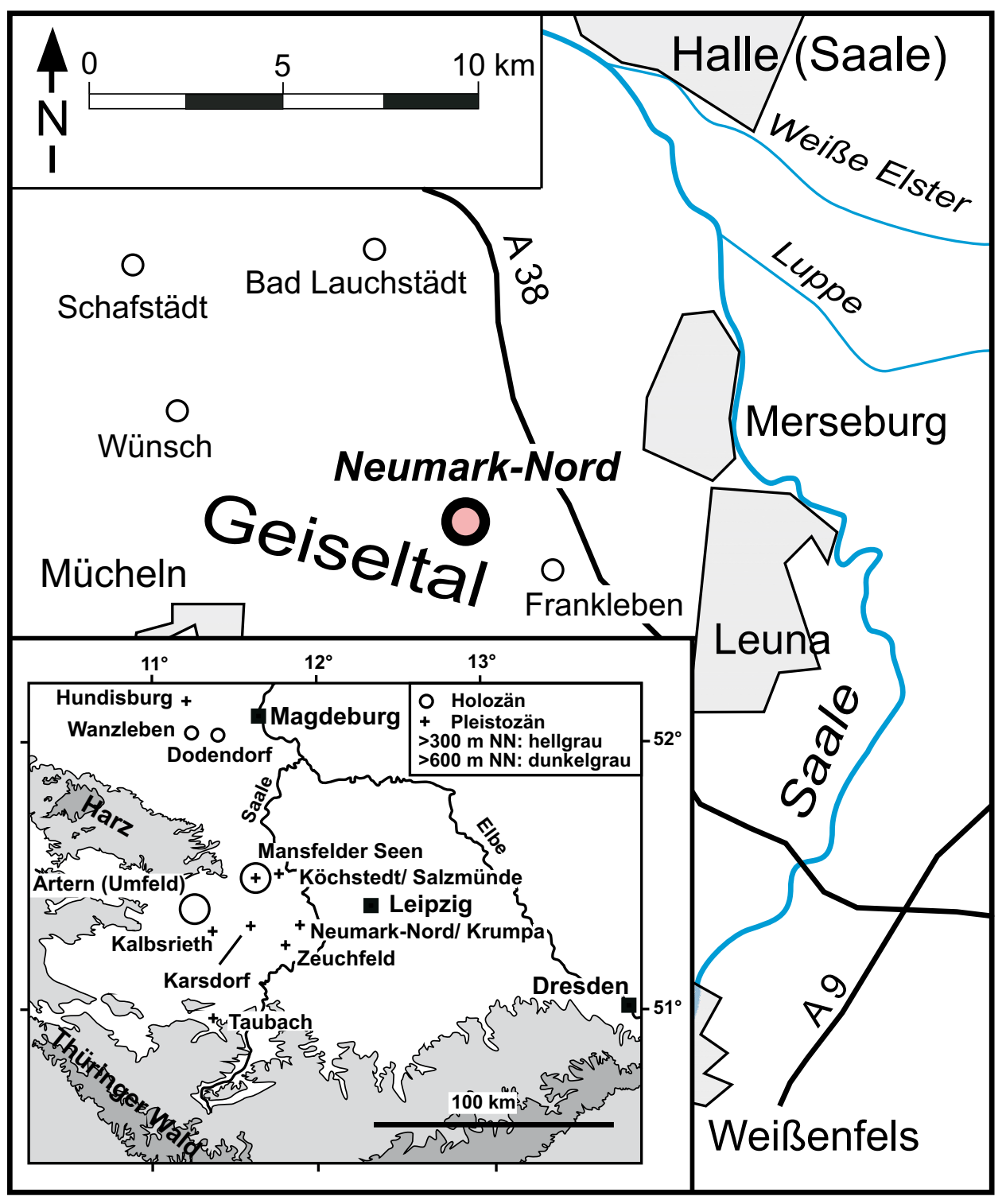

Abb. 1: Lage des Quartär-Aufschlusses Neumark-Nord und Ubersichtskarte von Mitteldeutschland mit pleistozänen und holozänen Vorkommen der Brackwasserschnecke cf. Hydrobia sp.

Fig. 1: Site of the Quaternary section of Neumark-North and map of Central Germany with Pleistocene and Holocene occurrences of the brackish water snail cf. Hydrobia sp.

\section{Inhaltsverzeichnis}

1 Einleitung (WANSA \& STRAHL)

2 Quartärgeologische Verhältnisse

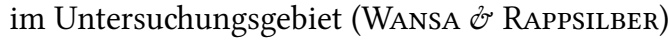

3 Methoden

3.1 Geländearbeiten

3.2 Analytik

4 Geologie und Paläontologie des Beckens NN2

4.1 Lagerungsverhältnisse (RAPPSILBER \& WANSA)

4.2 Liegendschichten am Beckenrand, Profil D

(WANSA)

4.3. Beckenzentrum, Hauptprofil A

4.3.1 Lithologie (WANSA)

4.3.2 Mineralogie und Geochemie (LUCKERT)

4.3.3 Palynologie (STRAHL)

4.3.3.1 Saale-Pleniglazial

4.3.3.2 Saale-Spätglazial

4.3.3.3 Eem-Interglazial

4.3.3.4 Weichsel-Frühglazial

4.3.4 Malakologie (MENG)
4.3.5 Kleinvertebraten (MENG)

4.4 Lithologie der Profile B1, B2 und C (WANSA) 150

4.5 Hangendschichten (WANSA)

5 Das Becken NN2 im Vergleich mit dem Becken NN1 151

5.1 Sedimentationsbedingungen

(WANSA, Luckert, MEng \& Strahl) 151

5.2 Palynostratigraphie (STRAHL) 153

5.3 Malakologische Interpretation (MENG) 156

6 Geochronologie

6.1 Lumineneszenzdatierungen an Sedimenten der Becken NN1 und NN2

(KRBETSCHEK \& ZÖLLER)

6.1.1 Diskussion der Daten von Karelin (1997)

6.1.2 Neue Datierungen

6.2 Aminosäuren-Geochronologie im Becken NN2

(MAchalett \& OCHES)

7 Fazit

9 Literatur 
1 Einleitung [S. Wansa \& J. Strahl]

Das wegen der überaus reichhaltigen Fossilfunde aus dem Mitteleozän weltberühmte Geiseltal südlich von Halle (Saale) (Abb. 1) steht seit Mitte der 1980er Jahre auch im Fokus der quartärgeologischen und archäologischen Forschung. Zwar sind die quartären Deckschichten der Braunkohle im vergangenen Jahrhundert wiederholt Gegenstand geologischer und paläontologischer Untersuchungen gewesen - u. a. wurde hier 1953 das Mammut-Skelett von Pfännerhall geborgen (ToEPFER 1957) und auch verschiedene warmzeitliche Ablagerungen sind bekannt geworden (Ruske 1961, MANIA \& MAI 1969) - doch setzte erst mit der Entdeckung eines interglazialen Seebeckens (Neumark-Nord 1, NN1) durch M. Thomae 1985 eine intensive und systematische Erforschung der komplexen quartären Schichtenfolge im östlichen Geiseltal ein. Eine interdisziplinäre Arbeitsgruppe unter Leitung von D. Mania hat die Beckensedimente in den Folgejahren detailliert dokumentiert und dabei umfangreiche Fossilfunde (darunter zahlreiche Skelette von Großsäugern) gesichert und ausgewertet. Mit dem Nachweis eines beachtlichen Inventars an Feuerstein-Artefakten wurde das Seebecken NN1 auch archäologisch interessant (MANIA 1990, 2004). Aufgrund verschiedener Indizien (u. a. Pollensukzession, Makroflora, Molluskenfauna, periglaziäres Deckschichten-Profil) soll die Beckenfüllung älter als die Eem-Warmzeit und jünger als die erste Saale-Vergletscherung sein (MANIA 1990, 1994). LiTt (1994a, b) entkräftete die Argumentation von Mania erstmals und ordnete NN1 dem Eem zu. Der Meinungsstreit war damit jedoch nicht beendet, sondern wurde durch weitere Untersuchungen an paläontologischem Fundmaterial aus NN1 (dargestellt u. a. in MANIA 2000 und MANiA et al. 2008) sowie durch die Entdeckung eines weiteren Interglazialbeckens (Neumark-Nord 2, NN2) durch D. Mania im Jahre 1995 neu befruchtet. NN2 liegt nur ca. 200 m nordöstlich von NN1 (Abb. 2). Laut der Aufschlussaufnahmen von D. Mania sollen die Beckensedimente von NN2 über die hangenden periglaziären Bildungen von NN1 hinweggreifen (MANIA et al. 2008, 2010).

Das Auftreten archäologischer Funde in verschiedenen Profilabschnitten von NN2 veranlasste das Landesamt für Denkmalpflege und Archäologie Sachsen-Anhalt (LDA) zu intensiven Grabungen und Profildokumentationen, die zunächst von D. Mania geleitet und maßgebend von T. Laurat und E. Brühl unter Mitwirkung zahlreicher Helfer realisiert wurden. Erste Ergebnisse sind bereits von LAURAT et al. (2004) vorgelegt sowie auf wissenschaftlichen Veranstaltungen des LDA im Oktober 2003 und August 2004 zur Diskussion gestellt worden. Ab 2006 unterstand die wissenschaftliche Koordination der archäologischen Arbeiten dem Römisch-

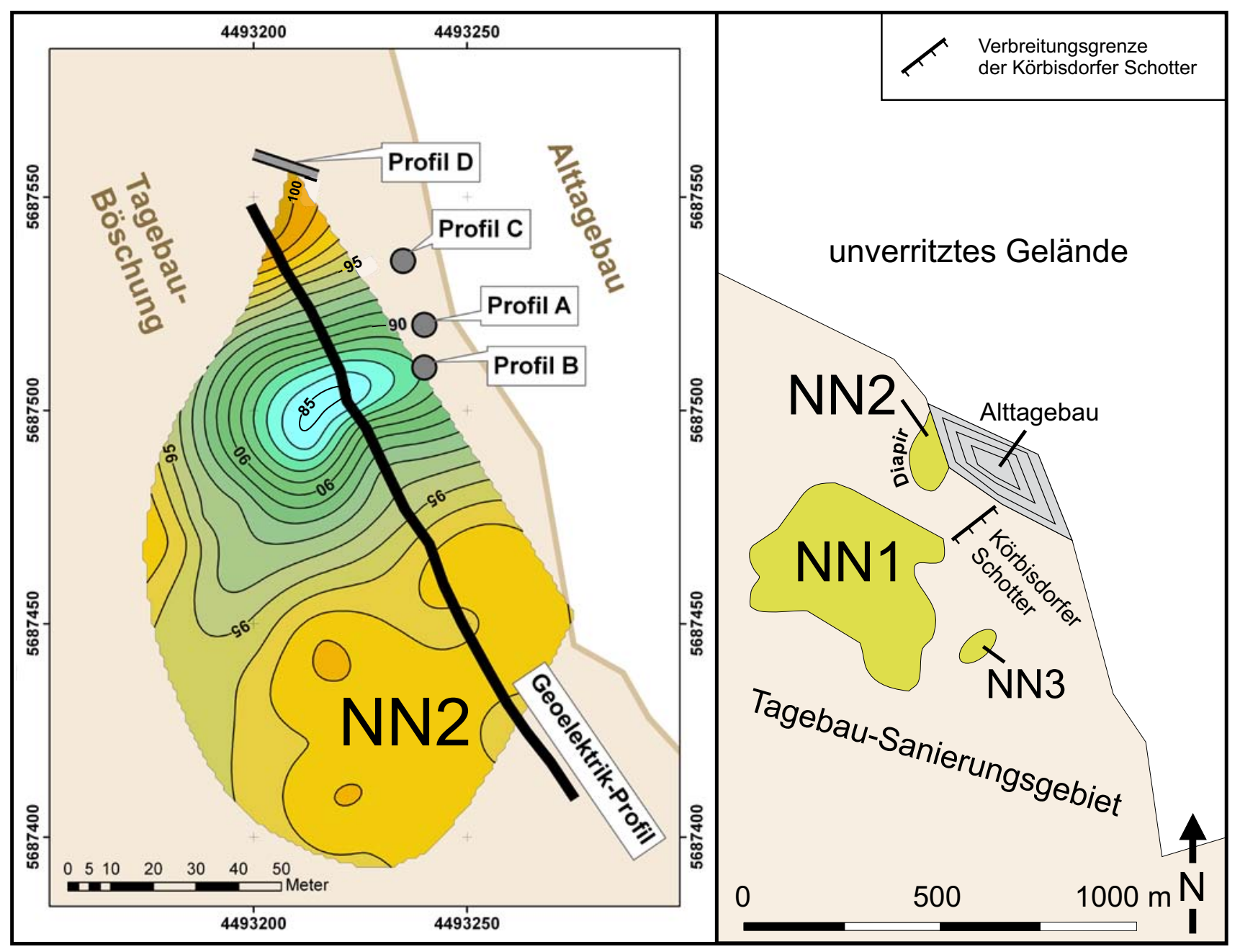

Abb. 2: Lage der Becken NN1, NN2 und NN3 sowie Isolinienplan der Unterkante der Beckensedimente in NN2 (nach THOMAE \& RAPPSILBER 2010, geändert). Fig. 2: Location map of the basins NN1, NN2 and NN3 as well as isoline map of the base of lacustrine sediments in basin NN2 (after THOMAE \& RAPPSILBER 2010, modified). 
Germanischen Zentralmuseum Mainz (RGZM). 2007 wurde vom LDA ein fast $7 \mathrm{~m}$ hohes Schurfprofil angelegt, das systematische Probennahmen für verschiedene Untersuchungsmethoden ermöglichte. Durch eine Rammkernsondierung im Rahmen eines Geländepraktikums des Instituts für Geowissenschaften der Martin-Luther-Universität Halle-Wittenberg (MLU) konnte die Profildokumentation im Liegenden bis zur Saale-Grundmoräne komplettiert werden. Nach Abschluss der Feldarbeiten 2008 wurde der Aufschluss verfüllt und das Grabungsgelände eingeebnet.

LAURAT et al. (2006) und MANIA et al. $(2008,2010)$ haben für NN2 ein mehrgliedriges klimatostratigraphisches Profil mit zwei Warmzeiten ausgewiesen, von denen die jüngere der Eem-Warmzeit entsprechen soll. In der Kompilation mit dem Becken NN1, das ebenfalls von der Ersten Saale-Grundmoräne unterlagert wird, sollen demnach in Neumark-Nord zwischen den saaleglaziären und den weichselzeitlichen periglaziären Ablagerungen limnische Sedimente aus insgesamt drei Warmzeiten überliefert sein. Schlussfolgernd stellen MANIA et al. (2008: 34) fest, dass die Zuweisung des gesamten saalezeitlichen Vereisungsgeschehens innerhalb einer Kaltzeit zum marinen SauerstoffIsotopenstadium (MIS) 6 nicht aufrechterhalten werden kann. Damit würde die Stratigraphie von Neumark-Nord in erheblichem Widerspruch zu den Belegen aus dem übrigen norddeutschen Vereisungsgebiet und darüber hinaus stehen, die Warmzeiten zwischen den saalezeitlichen Vergletscherungsphasen (Drenthe und Warthe) ausschließen (u. a. Ehlers et al. 2004, KüHner 2003, Meyer 2005, LITT et al. 2007). Zudem gilt heute aufgrund zuverlässiger Altersbestimmungen die Zuordnung des gesamten Saale-Hochglazials (Oberes Saale) zum MIS 6 als gesichert (u. a. Busschers et al. 2008, KrbetscheK et al. 2008). Weitere Thermomere interglazialen bzw. interstadialen Charakters datieren in das Untere Saale im Bereich des MIS 7 und sind hier aufgrund der Überlagerung durch drenthebzw. warthezeitliche Ablagerungen irrelevant für jegliche, sei es auch nur vergleichende Diskussion. In diesem $\mathrm{Zu}^{-}$ sammenhang ist gemäß der von SEIFERT-EuleN (2010: 271) vorgenommenen Vergleiche der warmzeitlichen Abfolge von NN1 mit der prä-eemzeitlichen Dömnitz- und sogenannten Uecker-Warmzeit (Profil Röpersdorf, ERD 1987) anzumerken, dass es sich bei letzterer um eemzeitliche und nicht intrasaalezeitliche Sedimente handelt (HERMSDORF \& Strahl 2006, LitT et al. 2007). Außerdem haben sich die von ERD (1987) publizierten Funde des Großen Algenfarns (Azolla filiculoides) anhand der Untersuchung neuer Bohrungen nicht bestätigt.

Hauptgegenstand vorliegender Publikation sind die Untersuchungsergebnisse einer Arbeitsgruppe, die von 2003 bis 2008 unter Koordination von S. Wansa (Landesamt für Geologie und Bergwesen Sachsen-Anhalt, LAGB) umfangreiches Probenmaterial aus dem Becken NN2 lithologisch, paläontologisch und chronometrisch analysiert hat. Außerdem soll die Beckenabfolge von NN2 vor allem im Hinblick auf die Stratigraphie ausführlich mit den Verhältnissen im Becken NN1 verglichen werden.

In die Bearbeitung des Beckens NN2 waren auch isotopen-geochemische Untersuchungen (T. Böttger, F. W. Junge) einbezogen, deren Ergebnisse separat publiziert werden sollen.

\section{Quartärgeologische Verhältnisse im \\ Untersuchungsgebiet [S. Wansa \& I. Rappsilber]}

Der Aufbau und die Lagerungsverhältnisse der quartären Schichtenfolge von Neumark-Nord sind in entscheidendem Maße durch Lagerungsdeformationen der Braunkohle geprägt, die bereits seit langem bekannt sind, aber erst von Thомае (1986) als durch autoplastisch-gravitative Ausgleichsbewegungen entstandene Braunkohlendiapire interpretiert wurden. Als Ursachen für die karbokinetischen Prozesse gelten die inverse Dichteschichtung (klastisches Sediment über Braunkohle) und die geringe innere Festigkeit der durch Permafrost beeinflussten, wassergesättigten Kohle (Thomae 1990, 2003). Nach Eissmann (1981) sind insbesondere Phasen des Permafrostzerfalls am Ende der Kaltzeiten für den Braunkohlendiapirismus prädestiniert. Im Geiseltal wurden Kohlediapire seit dem Spätelster in insgesamt vier Zeitabschnitten gebildet (Тномав 1990). Die oberflächennahe Lage der Kohle sowie ein relativ steiles Einfallen an der Neumark-Hauptschwelle (Prätertiär-Aufragung) dürften den Diapirismus in Neumark-Nord begünstigt haben (MANIA \& Thomae 1987). Die NE-SW-Orientierung der Diapire verläuft hier parallel zum Einfallen des Prätertiärs. Die Entstehung und Erhaltung der pleistozänen Seeablagerungen von Neumark-Nord ist an Randsenken von Braunkohlendiapiren gebunden. Aus der Randsenkenfüllung (Becken NN1) leiteten MAnia \& Thomae (1987: 39) ab, dass sich die Struktur „vermutlich langsam und stetig bis weit in das Interglazial hinein entwickelt" hat.

Aus der „Beobachtung“, dass einmal abgeschlossene gravitative Ausgleichsbewegungen kaum wieder aktiviert werden könnten, sondern sich in einer neuen Kaltzeit an anderer Stelle fortsetzten, schlussfolgerten MANiA et al. (2008), dass das Becken NN2 jünger sei als das Becken NN1. Dem steht entgegen, dass Braunkohlendiapire meist keine singulären Bildungen sind. In prädestinierten Arealen Mitteldeutschlands sind zahlreiche solikinetische Kohleaufragungen (Diapire, Kissen) mehr oder weniger synchron entstanden, aber auch die Mehrphasigkeit gravitativer Bewegungen an ein und derselben Struktur ist keinesfalls ungewöhnlich (siehe EISSMANN 1981, 1987) und wurde von Thomae (1990: 142) und Mania (2004: 37) sogar in direkten Zusammenhang mit der Anlage des Beckens NN1 gebracht. Den Überlegungen von MANIA et al. (2008), nach denen der Diapirismus von einer Bildungsphase zur nächsten räumlich wandert, so dass die Randsenkenfüllungen verschiedener Diapire verschieden alt sein müssten, kann daher nicht gefolgt werden.

Das Quartärprofil von Neumark-Nord beginnt mit den holstein- bis frühsaalezeitlichen Körbisdorfer Schottern der damals mit der Geisel vereinigten Unstrut (vgl. LAURAT et al. 2006). Die Schottermächtigkeit beträgt durchschnittlich $5 \mathrm{~m}$, in Randsenken können bis $15 \mathrm{~m}$ erreicht werden. Örtlich haben Kohlediapire den muschelkalkreichen Schotterkörper durchschlagen und die Schichtung verstellt. Im Untersuchungsgebiet (Abb. 2, rechts) keilen die Körbisdorfer Schotter an der SE-Flanke einer sattelförmigen Kohleaufragung aus, deren NW-Flanke das Becken NN2 im SE begrenzt (RAPPSILBER 2004a).

Der untere Teil der Körbisdorfer Schotter verzahnt randlich mit dem 1996 von D. Mania entdeckten und vor allem 


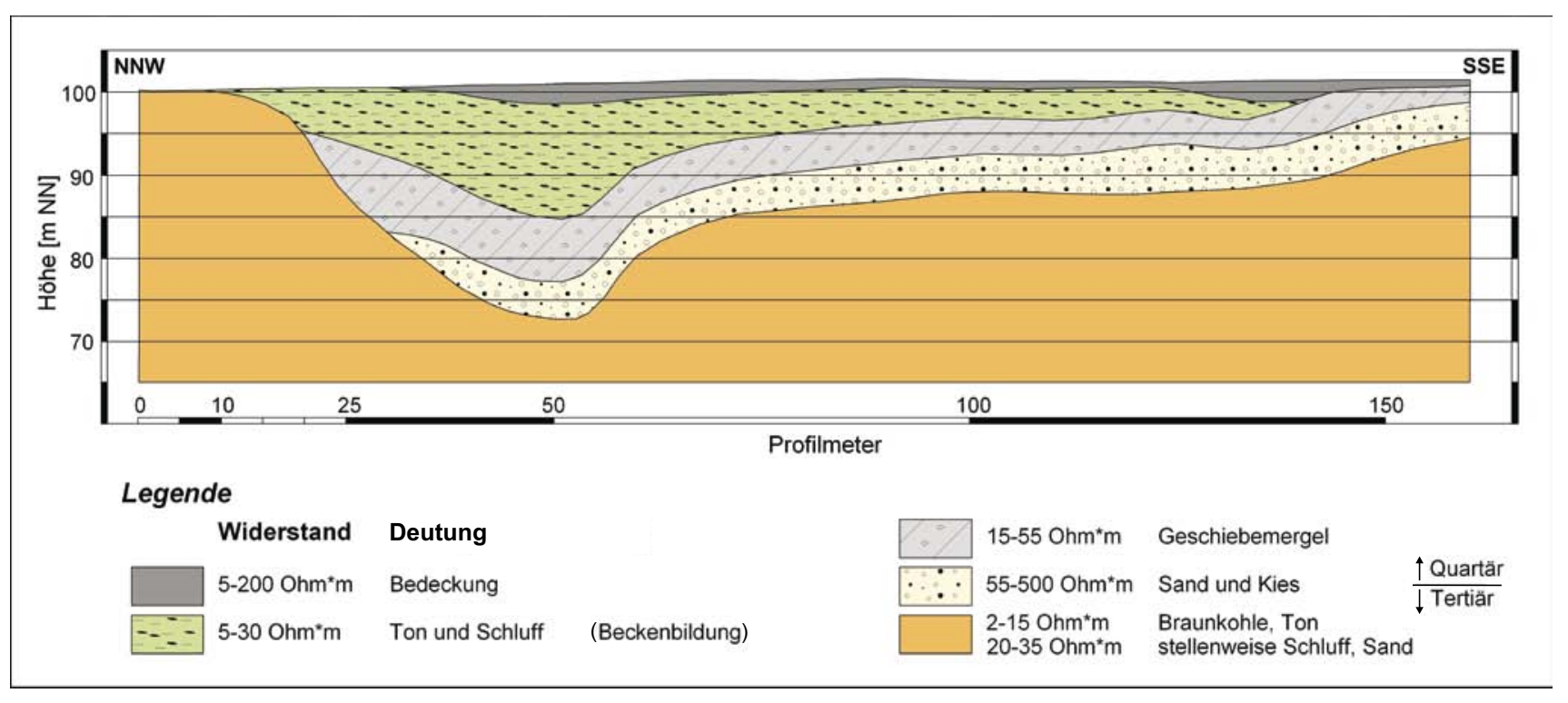

Abb. 3: Geoelektrischer Profilschnitt durch das Becken NN2 (THOMAE \& RAPPSILBER 2010).

Fig. 3: Geo-electric cross-section through the NN2 basin (THOMAE \& RAPPSILBER 2010).

2003 gut aufgeschlossenen Paläoseebecken Neumark-Nord 3 (NN3) (Abb. 2). Die Beckenfüllung besteht aus durch Diapirismus schräg gestellten, bis $7 \mathrm{~m}$ mächtigen Schluffen, die im unteren Teil Gehäuse von Valvata piscinalis piscinalis und darüber eine auffällige Konzentration zweiklappiger Schalen von Anodonta anatina aufwiesen (LAURAT et al. 2006, MANIA 2010b). Da diese Arten klimatisch weitgehend indifferent sind, gestattet ihr Vorkommen keine stratigraphische Interpretation ${ }^{1}$.

Über den Unstrut/Geisel-Schottern folgt eine ca. $5 \mathrm{~m}$ mächtige Grundmoräne, die nach geschiebeanalytischem Befund dem ersten Vorstoß des Saale-Inlandeises (ZeitzPhase) zuzuordnen ist (Thомав 1990). Nach einer kurzen Abschmelzphase (Pomßen-Intervall), in der sich der Eisrand bis in den Raum Petersberg - Landsberg - Delitzsch zurückverlagerte, stieß das Eis während der Leipzig-Phase erneut bis in das Geiseltal vor (EISSMANN 1975, LitT \& WANSA 2008), doch ist die Zweite Saale-Grundmoräne hier nur örtlich mit Mächtigkeiten bis ca. $2 \mathrm{~m}$ erhalten (Ruske 1961). Die Zeitz-Phase und die Leipzig-Phase sind die Vereisungsphasen des Drenthe-Stadiums im Saale-Elbe-Gebiet. Das jüngere Saale-Inlandeis (Warthe-Stadium bzw. Fläming-Phase) hat seine äußeren Randlagen weiter nördlich in der Colbitz-Letzlinger Heide und im Fläming, vielleicht auch in der Schmiedeberger Stauchendmoräne hinterlassen (zuletzt LiTT et al. 2007, LiTT ひ WANSA 2008).

Aufgrund des Kohlediapirismus ist die Erste Saale-Grundmoräne in ihrer Lagerung gestört. In den Randsenken haben sich über der Grundmoräne limnische Sedimente akkumuliert, die zum besonderen Gegenstand der Untersuchungen geworden sind. Im Hangenden lagert eine mächtige weichselzeitliche Schichtenfolge aus solifluidalen, limnischen, fluviatilen und äolischen Bildungen.

1 Das Becken NN3 wird von MANIA (2010b) als holsteinzeitlich bezeichnet. Dabei ist zu beachten, dass MANIA von einem „Holstein-Komplex“ ausgeht, der aus drei Warmzeiten und zwei Kaltzeiten besteht. Dies ist mit der gültigen Definition der Holstein-Warmzeit nicht vereinbar (siehe LiTT et al. 2007).

\section{Methoden}

\subsection{Geländearbeiten}

Zur Klärung der Lagerungsverhältnisse im Bereich des Beckens NN2 und seines engeren Umfeldes sind geophysikalische Messungen vorgenommen worden. Ein gravimetrisches Lokalfeld, das auf älteren Schweremessungen mit Punktabständen von 15-30 m beruht, lieferte einen ersten Überblick über die Strukturen der liegenden Braunkohle. Die Verbreitungsgrenze der Körbisdorfer Schotter wurde mit Hilfe von Georadarprofilen mit einer Gesamtlänge von $2500 \mathrm{~m}$ kartiert. Ausdehnung und Form des Beckens NN2 wurden durch geoelektrische Messungen bestimmt (Abb. 2 und 3). Insgesamt erfolgten 42 geoelektrische Tiefensondierungen in vier sich kreuzenden Profilen. Innerhalb der Profile betrug der Messpunktabstand zwischen 5 und 20 m. 10 Motorhammerbohrungen mit Tiefen bis zu $6 \mathrm{~m}$ dienten der Verifizierung der geophysikalischen Aussagen und stellten darüber hinaus eine Verbindung zum Becken NN1 her.

Im Mittelpunkt der Geländearbeiten stand die Dokumentation und Beprobung des oben angeführten, vom Landesamt für Denkmalpflege und Archäologie SachsenAnhalt (LDA) angelegten und eingemessenen Profils A (im Bereich des Hauptprofils 7 bei LAURAT et al. 2007), das durch eine im Juni 2007 niedergebrachte Rammkernsondierung (RKS) bis zur liegenden Grundmoräne erweitert wurde. Da die Sondierung von einer oberhalb der Schurfsohle gelegenen Berme aus niedergebracht wurde, überlappt der obere Teil des RKS-Profils mit dem unteren Teil des Schurfprofils (Abb. 4). Das Profil A (folgend auch als Hauptprofil A bezeichnet) befindet sich an der nördlichen Flanke unweit des Beckenzentrums (Abb. 2) und erschließt eine insgesamt 12,6 m mächtige Schichtenfolge, die im Liegenden mit 1,6 m Geschiebemergel beginnt und im Wesentlichen aus $11 \mathrm{~m}$ mächtigen, differenzierten Seeablagerungen besteht. 


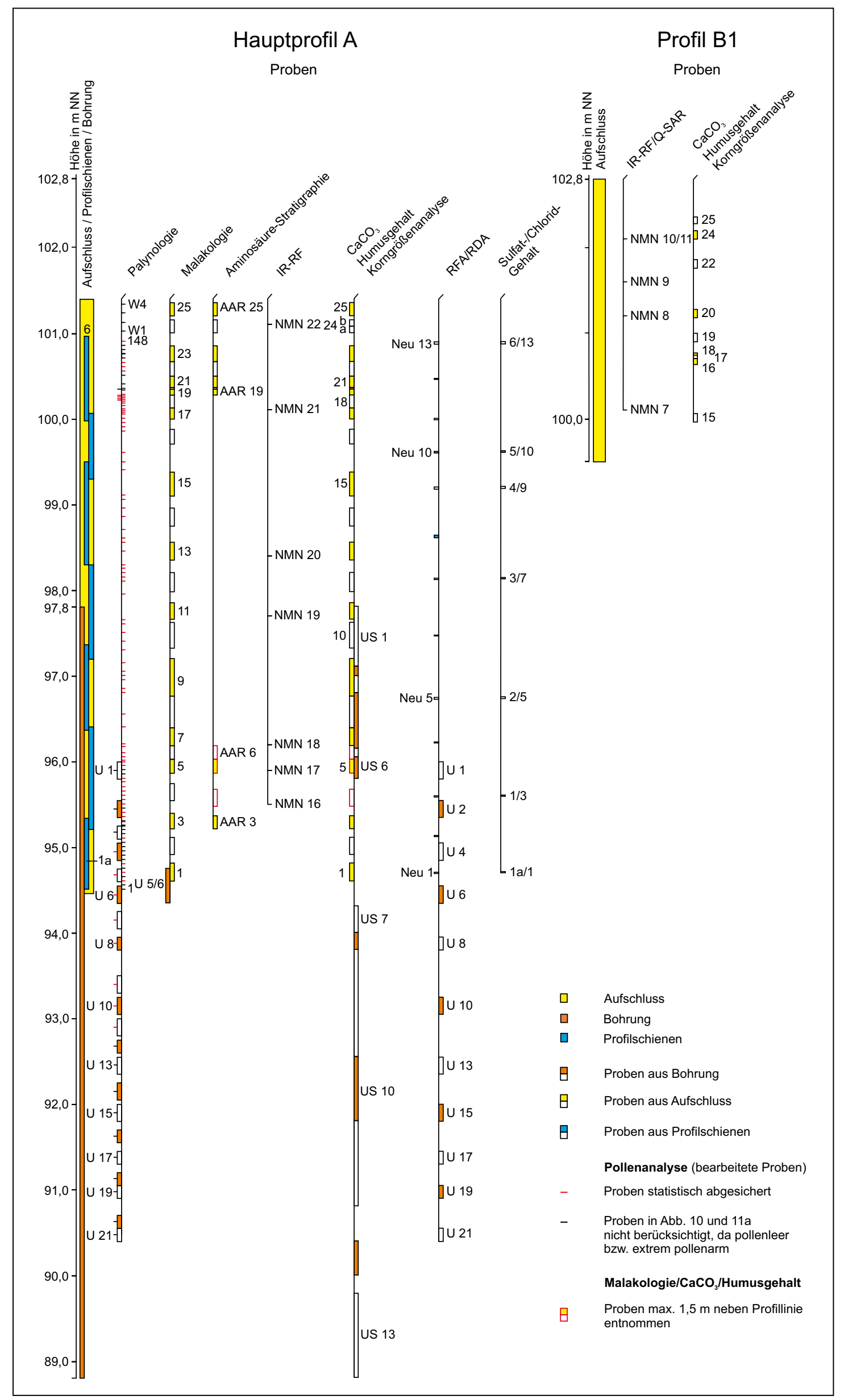

Abb. 4: Probenübersicht der Profile A und B1.

Fig. 4: Overview of samples from profiles $A$ and B1. 
Die gemeinsame Beprobung des Schurfprofils für die lithologischen, paläontologischen und chronometrischen Untersuchungen erfolgte im März und Mai 2007. Für die Pollenanalysen, die Röntgenfluoreszenz- und Röntgendiffraktionsanalysen sowie für geochemische Untersuchungen hinsichtlich Sulfat- und Chlorid-Gehalt wurde quasiungestörtes Material in U-Profilschienen entnommen. Um eine vollständige Beprobung des Profils zu gewährleisten, wurden die 0,8-1,2 $\mathrm{m}$ langen Schienen überlappend in die Schurfwand geschlagen (7 Profilschienen 1-6a, Abb. 4). Parallel dazu sind die Proben für die Korngrößenanalysen, Kalkgehalts- und Humusgehaltsbestimmungen gewonnen worden. Es handelt sich jeweils um Mischproben charakteristischer Profilabschnitte, wobei angestrebt wurde, alle wesentlichen makroskopisch erkennbaren Lithotypen $\mathrm{zu}$ erfassen. Aus den gleichen Profilabschnitten ist auch das Probenmaterial für die Bestimmung der Molluskenschalen gewonnen worden. Die Entnahmebereiche wurden dabei zur nachträglichen eindeutigen Parallelisierung der Proben (im Folgenden als „Pr.“ abgekürzt) untereinander auf den Rückseiten der Schienen markiert.

Die Probenmenge aus der RKS ist entsprechend den Erfordernissen der Analysenmethoden aufgeteilt worden. Für die Bestimmung des Molluskeninhalts wurde nur Material aus dem auffällig mit Schalenresten angereicherten, im Aufschluss etwa der Basis der Profilschiene 1a entsprechenden Abschnitt (Pr. U 5/6, Abb. 4) entnommen. Unter Verweis auf Abb. 4 sei vermerkt, dass die Proben aus der RKS mit „U“ bzw. „US“ (für die sedimentologischen Analysen) bezeichnet sind und im Gegensatz zu den Proben aus dem Schurfprofil von oben nach unten nummeriert wurden. Außerdem ist auf die z. T. doppelte Vergabe gleicher Bezeichnungen für verschiedene Proben hinzuweisen. Deshalb ist es wichtig, die Bezeichnungen generell im $\mathrm{Zu}^{-}$ sammenhang mit der entsprechenden Analysenmethode $\mathrm{zu}$ betrachten. Dadurch wird die eindeutige Zuordnung der im Text genannten Proben zu den Abbildungen und Tabellen gewährleistet. Die z. T. beidseitige Beschriftung der Probenskalen (Palynologie, Malakologie, Sedimentologie) wurde so zum einen aus Platzgründen und zum anderen zur besseren Unterscheidung von aus den Profilschienen bzw. aus der Bohrung gewonnenen Proben vorgenommen.

Im Dezember 2007 wurde die Probennahme im Hangenden des Hauptprofils A ergänzt (Pr. 23-25 Lithologie/Malakologie/Aminosäuren-Stratigraphie, Pr. W1-W4 Pollenanalyse). Außerdem wurde durch eine Ausschachtung auf der Sohle der Schurfgrube zusätzliches Probenmaterial aus dem Basisbereich gewonnen (Pr. 1 und 2).

Für die pollenstratigraphische Einstufung standen aus dem Hauptprofil A neben 148 Proben aus den sieben Profilschienen 1a-6, vier Einzelproben aus dem im Dezember 2007 geschaffenen Hangendaufschluss sowie 21 Proben aus der RKS zur Verfügung. Davon kamen 125 Proben zur pollenanalytischen Bearbeitung (Abb. 4), wovon 82 statistisch auswertbar waren und in das Pollendiagramm eingeflossen sind (Abb 10 und 11a). Generell beträgt der Probenabstand bei den Profilschienen $5 \mathrm{~cm}$. Lediglich bei markanten lithologischen Wechseln bzw. bei sehr gering anzunehmenden Sedimentationsraten wurde davon abgewichen und in deutlich engeren Abständen von minimal $1 \mathrm{~cm}$ beprobt. Bei den bereits im Gelände gewonnenen Proben aus dem Hangendauf- schluss sowie aus der RKS liegen wesentlich höhere Probenabstände, z. T. > 25 cm, vor (vgl. Abb. 4).

Für die Röntgendiffraktometeranalysen (RDA) wurden insgesamt etwa alle $0,5 \mathrm{~m} 26$ vor allem tonreichere Proben als Punktprobe (Profilschienen 1a-6) bzw. Schlitzprobe (RKS) im Labor entnommen; Kalkmudden und organogene Mudden blieben ausgespart.

Soweit für das Thema relevant, wird auf die seit 2003 erfolgte Bearbeitung weiterer, ebenfalls durch das LDA angelegter Schurfprofile im Bereich des Beckens NN2 Bezug genommen. Dazu gehört das ebenfalls beckenzentral gelegene Profil B1 (Abb. 4), das stratigraphisch im Wesentlichen dem oberen Teil von Profil A entspricht. Die Probennahme aus Profil B1 erfolgte im Oktober 2003. Dieses Schurfprofil wurde später um mehrere Meter vertieft, war jedoch zum Zeitpunkt der Aufnahme als Profil B2 im Juni 2004 bereits teilweise wieder verfüllt. Das an der nördlichen Beckenflanke gelegene Profile $\mathrm{C}$ wurde lediglich zur lithologischen Korrelation mit dem entsprechenden Abschnitt des Profils A dokumentiert. Das Profil D (Abb. 5) erschließt den unteren Profilabschnitt am nördlichen Beckenrand sowie die Liegendschichten des Beckens NN2.

Aus der limnischen Folge des Beckens NN2 sind in den Jahren 2003-2005 und 2007 insgesamt 50 Proben für malakologische Untersuchungen entnommen worden. Allein aus dem Hauptprofil A stammen 27 Proben mit über 200 Liter Sediment. Aus einem Teil der Proben wurde Material für die Aminosäuren-Datierung separiert. 2003 erfolgten zudem Probennahmen aus Sedimenten des Beckens NN1 für Lumineszenzdatierungen.

Die Lage der Profile wurde mit GPS ermittelt (GAussKRÜGER/BESSEL-Koordinaten in Abb. 2, links). Die Höhenangaben beziehen sich auf das von den archäologischen Bearbeitern vorgenommene Nivellement.

\subsection{Analytik}

Die Korngrößenzusammensetzungen, Kalk- und Humusgehalte bzw. Gehalte an organischer Substanz der Proben aus der Rammkernsondierung wurden im Labor des Instituts für Geowissenschaften der Martin-Luther-Universität Halle-Wittenberg (MLU) bestimmt, alle anderen im Labor des Landesamtes für Geologie und Bergwesen Sachsen-Anhalt (LAGB) in Halle. Die Korngrößenverteilungen sind mittels kombinierter Sieb- und Sedimentationsanalysen ermittelt worden. An der MLU wurden die Masse-Prozentgehalte von Kalk und der Glühverlust nach DIN 18129 bzw. DIN 18128 gewonnen. Zur Berechnung der organischen Substanz wurde der Karbonatgehalt vom Glühverlust abgetrennt (reduzierter Glühverlust). Im LAGB-Labor ist zur Bestimmung von Kalk und organischem Kohlenstoff ein Schwefel-Kohlenstoffanalysator verwendet worden. Die Kalkbestimmung erfolgte unter Zugabe von 50 \%-iger Phosphorsäure, der Kohlenstoff wurde nach DIN ISO 10694 ermittelt. Der Humusgehalt errechnet sich durch Multiplikation mit dem Faktor 1,724 aus dem Gehalt an organischem Kohlenstoff. Aus dem Becken NN2 (einschließlich des unterlagernden Geschiebemergels) sind insgesamt 90 Proben analysiert worden.

Die Kleingeschiebezusammensetzung der Grundmoräne wurde nach TGL 25232 ermittelt. Diese seit Jahrzehnten im Gebiet der ehemaligen DDR einheitlich angewendete Me- 
thode ermöglicht bei solider Interpretation der Zählergebnisse regionale lithostratigraphische Korrelationen. Für die Analyse der Geröllkomponenten fluviatiler Ablagerungen wurde die dafür häufig verwendete Fraktion 6,3-20 mm genutzt.

Für eine detaillierte mineralogische Bestimmung der Sedimente sind von jeder Probe (Gesamtprobe) und der Tonfraktion $(<2 \mu \mathrm{m})$ Röntgendiffraktometeranalysen (RDA) durchgeführt worden. Die Gewinnung der Tonfraktion erfolgte nach dem Atterberg-Verfahren. Die dabei vorher abgetrennte Sandfraktion $(>63 \mu \mathrm{m})$ wurde makroskopisch bewertet (Gehalte an Kies, organischer Substanz, Muschelschill, Glimmerschüppchen). Die Ermittlung der quantitativen Anteile der kristallinen Phasen erfolgte durch die Peakhöhenanalyse nach dem Verfahren mit Nickel als internem Standard bei drei Aufnahmen je Probe mit einem relativen Messfehler von $\leq 5 \%$. Die Bestimmung aller nachweisbaren kristallinen Phasen erfolgte anhand von Eichkurven bei einer Nachweisgrenze von ca. $1 \%$. Die Anteile an amorpher Phase können dabei nur indirekt als Rest zu $100 \%$ kristalliner Phase abgeleitet werden.

Für die Bestimmung der Tonminerale sind im Landeslabor Berlin-Brandenburg (LLBB) in Kleinmachnow Texturpräparate von der Tonfraktion $<2 \mu \mathrm{m}$ angefertigt worden, die im luftrockenen Zustand nach Behandlung mit Ethylenglykol (zum Nachweis quellfähiger Tonminerale) und nach Temperung bei $400{ }^{\circ} \mathrm{C}$ im Winkelbereich von $3-30^{\circ} 2 \theta$ geröntgt wurden. Die dabei erkennbaren charakteristischen Lage- und Formveränderungen bestimmter Tonmineralbasisreflexe ermöglichen genaue Aussagen über die qualitative und quantitative Zusammensetzung der Tonminerale bzw. bei Tonmineralen mit Wechsellagerungsstrukturen (mixedlayer-Minerale oder kurz ml-Minerale) zu Art, Anteil und Ordnungsgrad der vorhandenen Tonmineralschichten. Die Bestimmung der ml-Minerale und der Smektitschichtanteile in den ml-Mineralen erfolgte anhand der von REYNOLDS (1984) berechneten Modelldiffraktogramme.

Ergänzend wurden mittels Röntgenfluoreszensanalyse (RFA) die Haupt- und eine Reihe von Spurenelementen bestimmt.

Für die Pollenanalysen erfolgte die Aufbereitung der Proben aus dem Hauptprofil A im Pollenlabor des LLBB. Alle Proben wurden mit $\mathrm{HCl}, \mathrm{KOH}$ und nur z. T. HF sowie mit dem Acetolyse-Verfahren nach ERDTMAn behandelt. Außerdem wurden die Proben unter Verwendung eines $6 \mu \mathrm{m}-$ Siebes geschallt, um störende minerogene Feinpartikel zu entfernen.

Bei der palynologischen Bearbeitung kamen ausnahmslos Flüssigpräparate (Suspension) zur Anwendung. Basis der statistischen Auswertung der je Probe ermittelten Zählwerte ist die Grundsumme, die sich aus der Summe der Baum(BP) und Nichtbaumpollen (NBP): $\Sigma(\mathrm{BP}+\mathrm{NBP})=100 \%$ ergibt (i. d. R. ca. 333 bis maximal 400 Pollenkörner). Von dieser Grundsumme ausgenommen blieben alle unmittelbaren Lokalelemente, wie höhere Wasser- und Sumpfpflanzen, Farne und Moose sowie Reste von Algen, anderer Mikroorganismen und umgelagerte präquartäre Sporomorphen. Ihre Anteile wurden, wie die der einzelnen BP und NBP auf die Grundsumme bezogen. Da die Anteile präquartärer Sporomorphen in den Proben weit unter 100 \% lagen, wurde auch hier eine statistische Auswertung vorgenommen. Als Zähl- basis lag die wie oben beschrieben gebildete Grundsumme aller vermeintlichen quartären BP und NBP zugrunde.

Die Aufarbeitung der Proben (Schlämmen und Sieben) für die malakologischen Untersuchungen erfolgte hauptsächlich im LAGB Sachsen-Anhalt in Halle. Die verwendeten Siebe besitzen eine Maschenweite von 0,5 mm.

Die Methodik der Thermolumineszenz (TL)-Datierung im archäologischen Bereich (insbesondere Keramik) und erste Sedimentdatierungen reichen bis in die 1960er Jahre zurück. Eine breite Entwicklung von Lumineszenzmethoden für Sedimente erfolgte erst später, und die TL-Methode war bis in die 1990er Jahre für deren Altersbestimmungen am weitesten verbreitet. Erste Datierungen an Sedimenten von Neumark-Nord aus dieser Zeit basieren auf diesem Verfahren. TL-Datierungen an Sedimenten waren aber oft fehlerhaft, u. a. wegen der relativ langen Lichtexposition, die für die Einstellung eines TL-Nullpunktes erforderlich ist. Die vergangenen zwei Jahrzehnte sind durch die Entwicklung von Methoden der Optisch Stimulierten Lumineszenz (OSL) gekennzeichnet. OSL-Alter stützen sich auf sehr lichtempfindliche Lumineszenzsignale, die vom Zeitpunkt der letzten Lichtexposition von Quarz- oder Feldspat-Sedimentpartikeln bis zur Probennahme aufgebaut worden sind. Sehr präzise und zuverlässige Alter für die letzten ca. $100 \mathrm{ka}$, also von jungpleistozänen Sedimenten, sind vor allem durch spezielle Quarz-OSL-Datierungstechniken im neuen Jahrtausend möglich geworden. Mittelpleistozäne Sedimente können jedoch mit dieser OSL-Methode und auch mit IRSL (Infrarot Stimulierte Lumineszenz - eine spezielle Methode auf der Basis von Feldspäten) bisher nur unter bestimmten, selten erfüllten Bedingungen und nur ungenau datiert werden (Preusser et al. 2008). Verbesserungen in diesem Zeitbereich ermöglicht erst die im letzten Jahrzehnt entwickelte Infrarot-Radiofluoreszenzdatierung (TrautManN et al. 1999). Für weitere Informationen zur Lumineszenzdatierung allgemein und zu den im Folgenden beschriebenen Methoden und Problemen wird auf Preusser et al. (2008) und WintLe (2008) verwiesen.

Eine detaillierte Darstellung der gesamten neueren, auch in Neumark-Nord angewandten Datierungsanalytik findet sich in Degering \& KrbetscheK (2007a). Notwendige messtechnische Angaben können hier nur in Kurzform dargestellt werden:

Probennahme: Einschlagen (wasser- und) lichtdichter Plastik-Stechzylinder in frisch aufgeschlossene, möglichst homogene Sedimentschicht. Probe für Radionuklidanalyse aus unmittelbarer Umgebung.

Probenaufbereitung: lichtexponiertes Material der Frontund Rückseite für Wassergehaltsbestimmung; Siebung (verschiedene Kornfraktionen > $63 \mu \mathrm{m}$ ), Enfernung von Organik ( $\mathrm{H} 2 \mathrm{O} 2)$ und Karbonaten (verdünnte $\mathrm{HCl}$ ); Extraktion von Quarz und Kalifeldspat über Flotation und Dichtetrennung; HF-Ätzung (Alpha-Saum); abschließende Siebung. Wassergehalt: Natürliche Feuchte und Wassersättigungsgehalt. Die Prozedur der Sättigungsgehaltsbestimmung liefert nur einen Anhaltswert, der den wahren Wert i. A. etwas überschätzt, da die natürliche Packungsdichte dabei nicht erreicht wird. Vor allem bei stärker bindigem Material sind deshalb Korrekturen notwendig. Für die Proben konnte bis auf NMN 9 und NMN 10 (geringe Teufe) vor dem Tagebauaufschluss Lagerung im Grundwasserbereich angenommen 
werden. Wegen des Wasserhaltevermögens bindiger Sedimente ist für die Altersberechnung jedoch eher ein Wassergehalt repräsentativ, der näher in Richtung der natürlichen Feuchte als dem Labor-Sättigungswert mit eingeschränkter Aussage liegt. Für Datierungen an Kalifeldspat (IR-RF, IRSL) sind wegen des hohen Anteils der internen Dosisleistung $\left({ }^{40} \mathrm{~K},{ }^{87} \mathrm{Rb}\right)$ Wassergehaltsschwankungen wenig kritisch.

Radionuklidgehaltsbestimmung: Hochauflösende low-level HP-Ge Gammaspektrometrie, Analyse auf radioaktive Ungleichgewichte in der ${ }^{238} \mathrm{U}$-Reihe (außer N 3).

Paläodosisbestimmung:

1) Infrarot-Radiofluoreszenz (IR-RF): Kalifeldspat 100-160 $\mu \mathrm{m}$; Einzelpräparat (single-aliqout)-Messungen (5/6 mm, ca. 1-2 mg, Folie-Monolayer); IRSAR Messprotokoll (ERFURT \& KRBETSCHEK 2003).

2) Optisch Stimulierte Lumineszenz (OSL): Quarz (90-160 oder 160-200 $\mu \mathrm{m}$ ) feldspatfreie (IRSL-Test) Einzelpräparat (single-aliquot)-Messungen (4 mm Durchmesser, auf Al-Tellerchen, Silikon); Risoe DA-15 (480 nm LED, $90 \%$ Leistung, 7 mm U340 Detektionsfilter); SAR-Messprotokoll (Murray \& WintLe 2000).

3) Infrarot Optisch Stimulierte Lumineszenz (IRSL): Kalifeldspat (100-160 $\mu \mathrm{m})$, volumennormierte Präparate (ca. $4 \mathrm{mg}$ ), MAAD-Protokoll (WintLe 1997) (natürliche und 6 additive $\beta$-Dosen), thermische Behandlung $140^{\circ} \mathrm{C}, 48 \mathrm{~h}$; Risoe DA-12 (880 m LED, $8 \mathrm{~mW}$, Detektion (410 nm): BG39 + GG400 + BG3); Probe Neu 1: 0.5 s short-shine; N 3: 500 s shine-down, "late-light“ Subtraktion 400-500 s; fading-Test (> 3 Monate bei Raumtemperatur).

Kaliumgehalt Kalifeldspat: 12,5 \pm 0,5 \%; nach HunTLEy $\mho$ BARIL (1997): $95 \%$ Konfidenzintervall 11,5-13,5 \%; sowie langjährigen laborinternen Kontrollmessungen (nach DüTsCH \& Krbetschek 1997) der Probenaufbereitung.

Alters- und Fehlerberechnung: Software AGE (Neu 1, N 3) (Grün 1992); alle anderen mit Software ADELE (Kulig 2005). Prinzipien der Fehlerberechnung: Aitken (1985); Fehlerangaben: 1-sigma.

Die Aminosäuren-Geochronologie (folgend auch AAR - Amino Acid Racemization) basiert auf dem Maß der Razemisierung von Aminosäuren, die in den Kalkgehäusen von fossilen Gastropoden erhalten sind. Lebende Organismen produzieren Aminosäuren in der L-Form. Nach ihrem Ableben setzt ein Transformationsprozess (Razemisierung) ein, bei der die L-Form in die D-Form umgewandelt wird. Die Geschwindigkeit der Umwandlung von L- zu D-Aminosäuren ist vorranging eine Funktion der ab dem Ablagerungszeitraum vorherrschenden Temperaturentwicklung und der Zeit, die seit dem Ableben des Organismus verstrichen ist (Probenalter).

Die das Ausmaß der Razemisierung stark beeinflussende Temperaturentwicklung repräsentiert das kinetische Mittel aller Temperaturen die eine Probe seit ihrer Ablagerung erfahren hat (effektive diagenetische Temperatur). Da diese auf quartären Zeitskalen stattfindenden Temperaturhistorien im seltensten Fall bekannt sind bzw. keinesfalls mit einer für geochronologische Methoden adäquaten Präzision rekonstruierbar sind, hat es sich zunehmend durchgesetzt, AAR als eine relative Datierungsmethode anzuwenden, bei der keine Kenntnis über die Paläo-Temperaturentwicklung notwendig ist (WeHMiller \& Miller, 2000).
Die Annahme hierbei ist, dass Proben aus einem begrenzten geographischen Raum und einer Höhenzone mit vergleichbaren heutigen mittleren Jahrestemperaturen (z. B. Mitteldeutschland mit Ausnahme der Mittelgebirgshochlagen), auch eine vergleichbare oder ähnliche Temperaturhistorie seit der Ablagerung erfahren haben (mittlere Jahrestemperatur, MAT $+/-1^{\circ} \mathrm{C}$, Temperaturamplitude an der Beprobungslokalität $\leq 6^{\circ} \mathrm{C}$ ). Unter dieser Vorrausetzung können Unterschiede im D/L-Verhältnis von Proben aus einer geographischen Region exklusiv als ein Maß für das relative Alter der Probe interpretiert werden - Proben mit ähnlichen D/L-Werten haben das gleiche geochronologische Alter, Proben mit höheren D/L-Werten sind älter, Proben mit niedrigeren $\mathrm{D} / \mathrm{L}$-Verhältnissen entstammen einem jüngeren Ablagerungszeitraum. Sofern für eine oder mehrere Aufschlüsse in dieser Region eine unabhängige Alterskontrolle (radiometrisch, biostratigraphisch, stratigraphisch) vorliegt, können die D/L-Werte als ein höchst verlässliches Werkzeug zum Überprüfen bestehender geochronologischer Korrelationen und zum Etablieren regional-geochronologischer Netzwerke verwendet werden (WEHMILLER \& Miller 2000). Für diese Anwendung der AAR als relative, stratigraphische Datierungsmethode hat sich in der englischsprachigen Literatur der Begriff „aminostratigraphy“ durchgesetzt (im Kontrast zu „aminochronology“), so dass im Folgenden auch der Begriff Aminosäuren-Stratigraphie benutzt wird.

Neben den Faktoren Temperatur und Zeit, wird das Ausmaß der Razemisierung, wenn auch untergeordnet, durch die Taxonometrie der Gastropoden und, insbesondere bei stark basischen Umweltbedingungen, durch den $\mathrm{pH}$-Wert des Ablagerungsmilieus bestimmt. Proben aus stark basischen Umweltmilieus $(\mathrm{pH}>9)$ werden daher nicht für Analysen zur Aminosäuren-Stratigraphie herangezogen. Um den Einfluss taxonomischer Faktoren auf die Razemisierung auszuschließen, konzentrieren sich aminostratigraphische Untersuchungen taxonomisch auf Probenabfolgen einer einzelnen Gattung bzw. sogar Art (z. B. Gyraulus laevis), deren fossile Kalkgehäuse abundant in möglichst vielen stratigraphischen Horizonten bzw. in zu vergleichenden geologischen Profilen vorkommen. Unter der Voraussetzung, dass eine solche monogenerische Probenabfolge eine ähnliche Temperaturentwicklung seit der Ablagerung erfahren hat, ist das Verhältnis von D- zu LAminosäuren (D/L ratio) ein Maß zur Bestimmung des relativen Alters des beprobten Materials (vgl. oben).

Abschließend bleibt zu bemerken, dass bei aminostratigraphischen Untersuchungen generell versucht wird, Beprobungshorizonte auszuwählen, die durch einheitliche Sedimentationsmilieus geprägt sind und keine zusätzliche Temperaturhistorie durch den Einfluss starker täglicher oder saisonaler Temperaturschwankungen (im Laufe der Paläo-Temperaturentwicklung) bzw. durch wechselnde Umweltbedingungen erfahren haben. Ein ideales System stellen z. B. mächtige Löss-Paläobodensequenzen dar, bei denen sich die aminostratigraphische Beprobung auf die kaltzeitlich abgelagerten Lösse beschränkt. Warmzeitliche Sedimentabfolgen sind oft durch geringere Sedimentationsraten, Sedimentationsunterbrechungen oder, wie beim Paläoseebecken NN2, durch Seespiegeländerungen inklusive Verlandungstendenzen geprägt. Solche wechselnden 
Paläoumweltbedingungen können eine zusätzliche Temperaturhistorie induzieren, die das AAR-Signal beeinflussen kann. Die erfolgreiche Anwendung der Aminosäuren-Stratigraphie wird dadurch nicht ausgeschlossen, es ist aber mit breiteren Fehlerwerten zu rechnen. Eine detaillierte Beschreibung aminogeochronologischer Voraussetzungen und Anwendungen legten Wehmiller \& Miller (2000) vor.

In Neumark-Nord wurden für geochronologische Untersuchungen mittels AAR Kalkschalen fossiler Gastropoden der Arten Anisus leucostoma und Gyraulus laevis genutzt. Nach einer intensiven Reinigung und Aufbereitung des Probenmaterials erfolgten die Messungen unter Verwendung eines Hochdruckflüssigchromatographen (revers phasedHPLC) im Amino Acid Geochronology Labor an der Bentley Universität (Massachusetts, USA) nach der von KAUFMAN \& MANLEy (1998) beschriebenen Methodik. Für alle Proben wurde standardmäßig eine Auswahl von Aminosäuren gemessen und analysiert - D/L-Asparaginsäure, Glutaminsäure, Phenylalanin, Valin und alloIsoleucin/Isoleucin - wobei sich die hier vorgestellten Ergebnisse auf D/L-Glutaminsäure konzentrieren, da diese Aminosäure konsistent Ergebnisse mit einer hohen Auflösung und Reproduzierbarkeit lieferte und sich besonders für relative Altersbestimmungen an alten Proben (Glazial-Interglazial-Skala) eignet (vgl. KAufmAN \& MANLEY 1998).

Während aminostratigraphische Untersuchungen erfolgreich an Lösssequenzen in Nordamerika und Europa angewandt wurden (OcHes \& McCoy 1995, 2001, OcHes et al. 1996, 2000) und wesentlich zur geochronologischen Validierung zentralasiatischer Lössablagerungen beitrugen (Machalett et al. 2008, Machalett 2010), werden hier erstmals Ergebnisse aminostratigraphischer Arbeiten an quartären Ablagerungen Mitteldeutschlands präsentiert.

\section{Geologie und Paläontologie des Beckens NN2}

\subsection{Lagerungsverhältnisse [I. Rappsilber \& S. Wansa]}

Geoelektrische Messungen flankiert von Rammkernsondierungen haben gezeigt, dass sich das Becken NN2 in der Randsenke eines bogenförmig verlaufenden Kohlediapirs entwickelt hat (RAPPSILBER 2004b). Der Diapir begrenzt die Randsenke bzw. das Becken im NW und W. Seine Flanke (Kohleoberfläche) fällt mit ca. $40^{\circ}$ zum Becken hin ein (Abb. 2 und 3). Im S und SE ist ebenfalls ein Anstieg der Kohleoberfläche $\mathrm{zu}$ verzeichnen, der im unteren Teil sogar Neigungswinkel von ca. $50^{\circ}$ erreichen kann, nach oben aber rasch flacher wird. Nach dem geoelektrisch ermittelten Isolinienplan (Abb. 2) fällt die Basis der Beckensedimente (bzw. die Geschiebemergeloberfläche) im NW und W mit ca. $25-30^{\circ}$ ein. Hingegen ist im unteren Abschnitt der S-Flanke örtlich mit Neigungswinkeln bis $40^{\circ} \mathrm{zu}$ rechnen, die zum Beckenrand hin stark abnehmen (Abb. 3). Das Becken ist somit asymmetrisch ausgebildet, wobei die größten Mächtigkeiten (bis ca. $16 \mathrm{~m}$ ) in einem SW-NE orientierten Bereich nahe dem Diapir erreicht werden. Südlich davon ist die Beckenfüllung meist weniger als $5 \mathrm{~m}$ mächtig und keilt flach aus. Während die Saale-Grundmoräne sowie die geoelektrisch dokumentierten unterlagernden Sande und Kiese (tertiäre Quarzschotter nach freundl. mündl. Mitt. von M. Thомав) das Relief der Kohleoberfläche im We- sentlichen nur nachzeichnen, hat die limnische Sedimentation eine weitgehende Nivellierung des Beckens bewirkt.

Die Isolinienführung der Unterkante der Beckensedimente in Abb. 2 wurde gegenüber der Darstellung bei TноMAE \& RAPPSILBER (2010) leicht verändert. Einzelne geoelektrische Messungen nordöstlich der eingetragenen Profillinie waren durch die Ringleitung des Tagebaus gestört und ergaben kein eindeutiges Schichtmodell. Inzwischen sind aber in diesem Bereich zusätzlich die Profile A und D berücksichtigt worden.

Im SW wird der Beckenrand von der flachen Böschung des Tagebaurestloches Neumark-Nord gekappt, im NE endet die Verbreitung abrupt an einer alten Tagebaukante. Zudem hat ein Grabenaufschluss gezeigt, dass die Beckensedimente im N diskordant von Fließerde abgeschnitten werden (4.5). Die erhaltene Fläche von NN2 umfasst somit noch reichlich 1 ha (Abb. 2).

\subsection{Liegendschichten am Beckenrand, Profil D [S. Wansa]}

Das Liegende der limnischen Sedimente bildet eine ca. $5 \mathrm{~m}$ mächtige Grundmoräne (RAPPSILBER 2004b), die in einem Grabenaufschluss am Beckenrand unmittelbar an der hier mit ca. $25^{\circ}$ einfallenden Flanke des Kohlediapirs dokumentiert werden konnte (Abb. 2 und 5). Die Grenzfläche zum Liegenden bildet eine dünne Kieslage in sandiger, z. T. schluffiger Matrix. Mehr als 90 \% der Gerölle sind gerundete Quarze. Daneben kommen Quarzite, Porphyre und Feuersteine vor. Die Grundmoräne ist infolge des Diapirismus schräg gestellt und besteht aus einem heterogen aufgebauten Geschiebemergel mit nahezu gestreckter Kornsummenkurve (geschiebereiches Sand-Schluff-Gemisch mit knapp $20 \%$ Ton). Der meist klüftige Diamikt ist im unteren Teil massig ausgebildet und dunkelgrau gefärbt. Linsen von Sand oder kiesigem Sand treten vornehmlich im oberen, graubraunen Bereich auf; sie lassen die gleiche Verstellung wie der gesamte Moränenkörper erkennen. Außerdem waren einzelne adäquat orientierte, z. T. mit Sand gefüllte Scherfugen zu beobachten. Die Kalkgehalte der Matrix betragen $6 \%$ im unteren und $9 \%$ im oberen Teil (vgl. Abb. 5: Pr. G1 und G2).

Das Alter der Grundmoräne ergibt sich sowohl aus der im Umfeld dokumentierten Überlagerung der holstein- bis frühsaalezeitlichen Körbisdorfer Schotter als auch aus der Geschiebezusammensetzung (Abb. 5). Die relativ geringen Quarzgehalte, die überwiegend eckigen bis kantengerundeten Porphyre sowie hohe Anteile von abgerollten und verwitterten Geschieben in der Feuerstein-Gruppe kennzeichnen die Moräne als saalezeitlich (vgl. Ruske 1961, Schulz 1962, Wansa \& Radzinski 2004). Die Geschiebegruppen Quarz, Porphyr und Sandstein/Quarzit spiegeln vorwiegend die Aufnahme von Lokalmaterial in den Gletscher wider. Die Porphyre lassen sich zum größten Teil dem Halleschen Vulkanitkomplex zuordnen, einzelne stammen aus dem Thüringer Wald und wurden aus Saale- bzw. Unstrut-Schottern aufgenommen. Die Kalkstein-Gruppe wird stark von nordischem, paläozoischem Kalkstein dominiert, wobei die Unterscheidung von einheimischem Muschelkalk nicht immer ganz eindeutig ist. Für die Einordnung der Grundmoräne innerhalb des Drenthe-Stadiums des Saale-Komplexes sind vor allem die Anteile an Nordischem Kristallin und Quarz ausschlaggebend. Danach handelt es sich hier um die Erste Saale-Grundmoräne (Zeitz-Phase), die sich gegenüber der 


$$
\text { ESE }
$$

$\mathrm{mNN}$

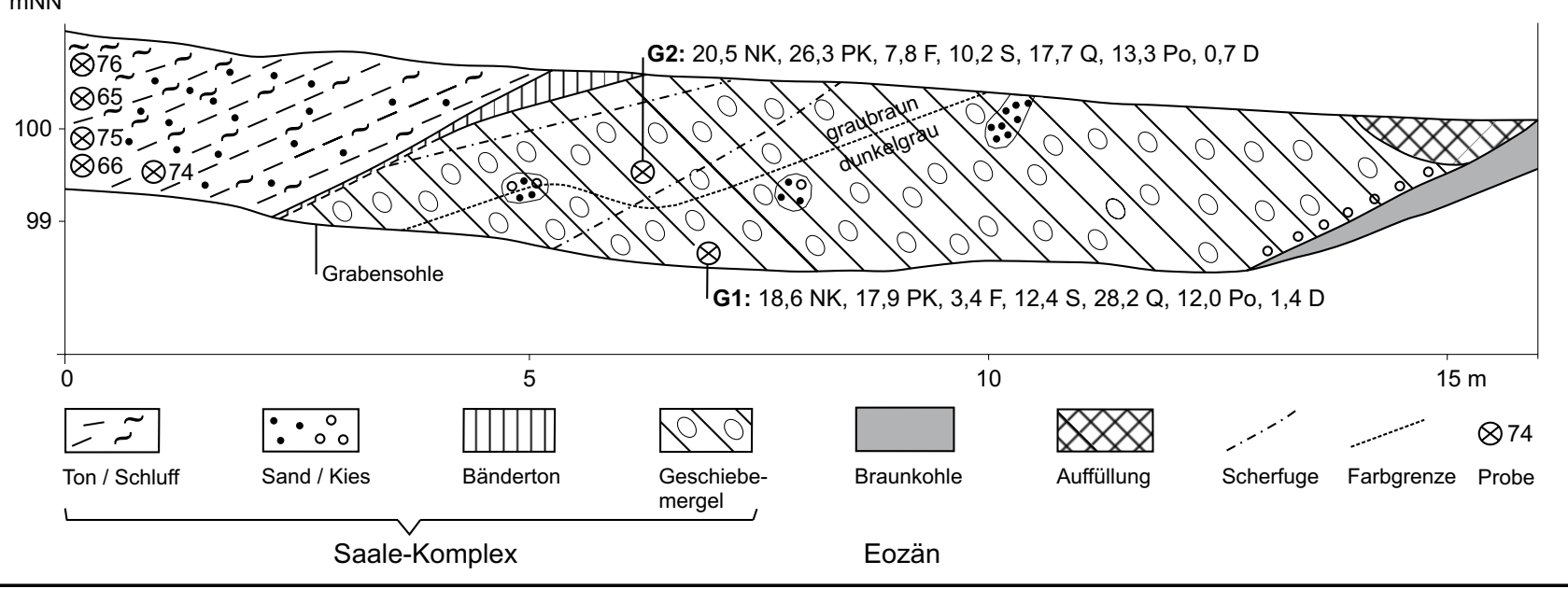

Abb. 5: Profil D, Aufnahme Juni 2004. Geschiebezusammensetzung in Korn-\% (Fraktion 4-10 mm): NK - nordisches Kristallin, PK - paläozoischer Kalkstein, F-Feuerstein, S - Sandstein, Quarzit, Q - Quarz, Po - einheimischer Porphyr, D - Dolomit.

Fig. 5: Profile D, documentation fune 2004. Gravel assemblages of the till (grain-size range 4-10 mm, grain-\%): NK - nordic crystalline, PK - paleozoic limestone, F-flint stone, $S$ - sandstone, quartzite, $Q$ - quartz, Po - local porphyry, D - dolomite.

Zweiten Saale-Grundmoräne (Leipzig-Phase) durch meist höhere Quarz-Werte bei geringeren Kristallin-Anteilen auszeichnet (vgl. EIssmann 1975). Auch die deutliche PorphyrKomponente stützt die Zuordnung zum ersten Saale-Eisvorstoß (vgl. Ruske 1961). Am Rande sei erwähnt, dass eine von der Basis des Beckens NN1 entnommene Geschiebemergelprobe annähernd die gleiche Geschiebezusammensetzung aufweist wie die Proben G1 und G2 (Abb. 5). Damit wird die bereits von THомав (1990) vorgenommene Stratifizierung der Grundmoräne im Liegenden des Beckens NN1 als Erste SaaleGrundmoräne untermauert. Nach der aktuellen lithostratigraphischen Definition gehört sie zur Zeitz-Glaziär-Formation (WANSA 2008).

Im Aufschluss wird die Grundmoräne von max. $30 \mathrm{~cm}$ deformiertem Bänderton überlagert (Unterer BruckdorferBänderton nach Junge 1998), der dicht über der Grabensohle bis auf 1-2 cm bzw. 5 dünne Warven reduziert ist. Wahrscheinlich steht die Fältelung und partielle Erosion des Bändertons mit dem Zweiten Saale-Eisvorstoß in Verbindung, doch ist dies hier nicht belegbar, da unmittelbar im Hangenden die limnische Sedimentfolge des Beckens NN2 beginnt. Der Bänderton wird von dunkelbraungrauem stark schluffigem, sandigem Ton überlagert (Pr. 74), der zum Hangenden in graubraunen schluffig-sandigen Ton (Pr. 66 und 75) und hellgraubraunen schwach tonigen bis tonigen Schluff (Pr. 65 und 76) übergeht, welcher aufgrund des auffälligen Grobschluffmaximums von $>50 \%$ schwemmlössartig erscheint (Abb. 5). Die Kalkgehalte der Seetone und -schluffe ${ }^{2}$ betragen 6-11\%, die Humusgehalte erreichen nur knapp $1 \%$.

Wenige Meter östlich des Aufschlussgrabens befand sich in feinsandigem Grobschluff (im Hangenden der beschriebenen Beckensedimente) der archäologische Hauptfundhorizont NN2/2 (LAURAT et al. 2006), der hier Gegenstand malakologischer (5.3) und aminostratigraphischer (6.2) Untersuchungen war.

2 Diese petrogenetischen Bezeichnungen gelten laut Geologischer Kartieranleitung (Ad-hoc-Arbeitsgruppe Geologie) für klastische nichtglaziäre Seeablagerungen.

\subsection{Beckenzentrum, Hauptprofil A}

\subsubsection{Lithologie [S. Wansa]}

Die Erste Saale-Grundmoräne ist in den unteren 1,6 m der Rammkernsondierung zwischen 90,4-88,8 m NN (Abb. 6) erreicht worden. Sie besteht hier aus einem olivgrauen Geschiebemergel mit einer $20 \mathrm{~cm}$ mächtigen bräunlich-grauen Toneinlagerung. Die Korngrößenzusammensetzung des Geschiebemergels entspricht weitgehend der im Aufschlussgraben am Kohlediapir (4.2), weist aber nur max. $10 \%$ Ton auf (Pr. US 12 und US 13, Abb. 6). Mit deutlicher Grenze setzt über der Grundmoräne die limnische Schichtenfolge ein. Sie beginnt mit einem braungrauen Sediment, das zu fast gleichen Teilen aus Ton, Schluff und Sand besteht (Pr. US 11). Zum Hangenden steigt zunächst der Sandgehalt auf Kosten des Schluffanteils auf fast $40 \%$ an (Pr. US 10), und danach ist ein deutlicher Wechsel zu einem braungrauen bis graubraunen Grobschluff- und Feinsand-dominierten Korngemisch mit sehr geringem Tongehalt festzustellen (Pr. US 9). Somit zeichnen sich zu Beginn der limnischen Sedimentation tendenziell ähnliche Entwicklungen der Korngrößenzusammensetzung im Zentrum und am Rand des Beckens ab (vgl. 4.2). Zum Hangenden verschiebt sich das Maximum in den Mittel- bis Grobschluff-Bereich (Pr. US 1, US 3, US 4, US 6-8). Die (Fein-)Sandanteile des Seeschluffs liegen meist unter $20 \%$, die Tongehalte variieren zwischen 5 und $28 \%$ (Pr. US 1-4). Vereinzelt treten stark sandige Schlufflagen auf (Pr. US 2, US 5).

Das Schurfprofil des Tagesaufschlusses umfasst eine knapp 7 m mächtige Sedimentfolge zwischen 101,4 und 94,5 m NN (Abb. 4, 6 und 7). Der basale Teil wurde durch eine Ausschachtung auf der Sohle des Schurfprofiles freigelegt. Damit wurde eine Uberlappung mit dem oberen Teil des Profils aus der Rammkernsondierung um insgesamt 3,3 m ermöglicht (97,8-94,5 m NN).

Das Profil beginnt im Liegenden mit mehr oder minder deutlich laminiertem tonigem bis schwach tonigem Schluff 
Hauptprofil A

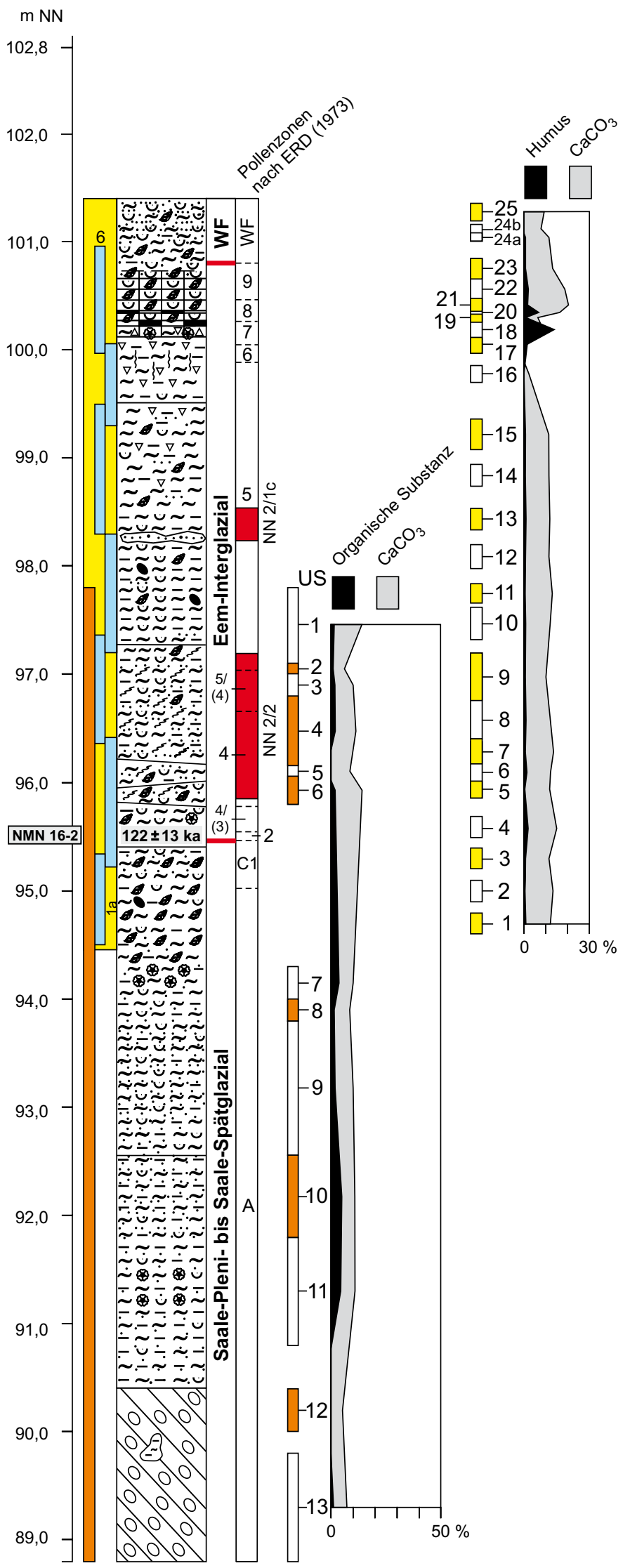

\section{Profil B1}

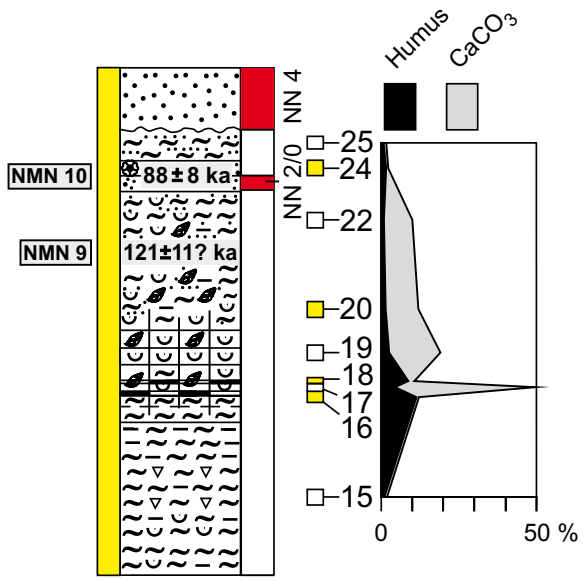

NMN 9 Lumineszenz-Datierungsprobe

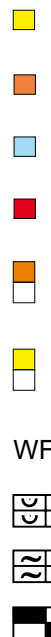

Aufschluss

$\square \quad$ Bohrung

$\square \quad$ Teilprofile (=Profilschienen)

$\square \quad$ Archäologische Fundschichten

Q Proben aus Bohrung

Proben aus Aufschluss

WF Weichsel-Frühglazial

Uy Kalkmudde

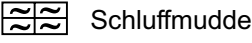

$\square$ Detritusmudde/Algenmudde

$\sim \sim$ Schluff, kalkhaltig

$\sim$ Schluff, tonig

$\widetilde{\sim}$ Schluff, tonig, kalkhaltig

$\sim \approx$ Schluff, feinsandig, tonig

$\sim \dot{\sim}$ Schluff, feinsandig bis grobsandig

Do\ Geschiebemergel

$\because:$ Sand, schwach kiesig

$\bullet \bullet$ Kies, sandig

... Sandlagen, -schlieren

Fließtexturen

- Mollusken

Pflanzenreste

\} Wurzelröhren

$\nabla \quad$ Gips

$\Delta \quad$ Vivianit

- Kalkkonkretionen 


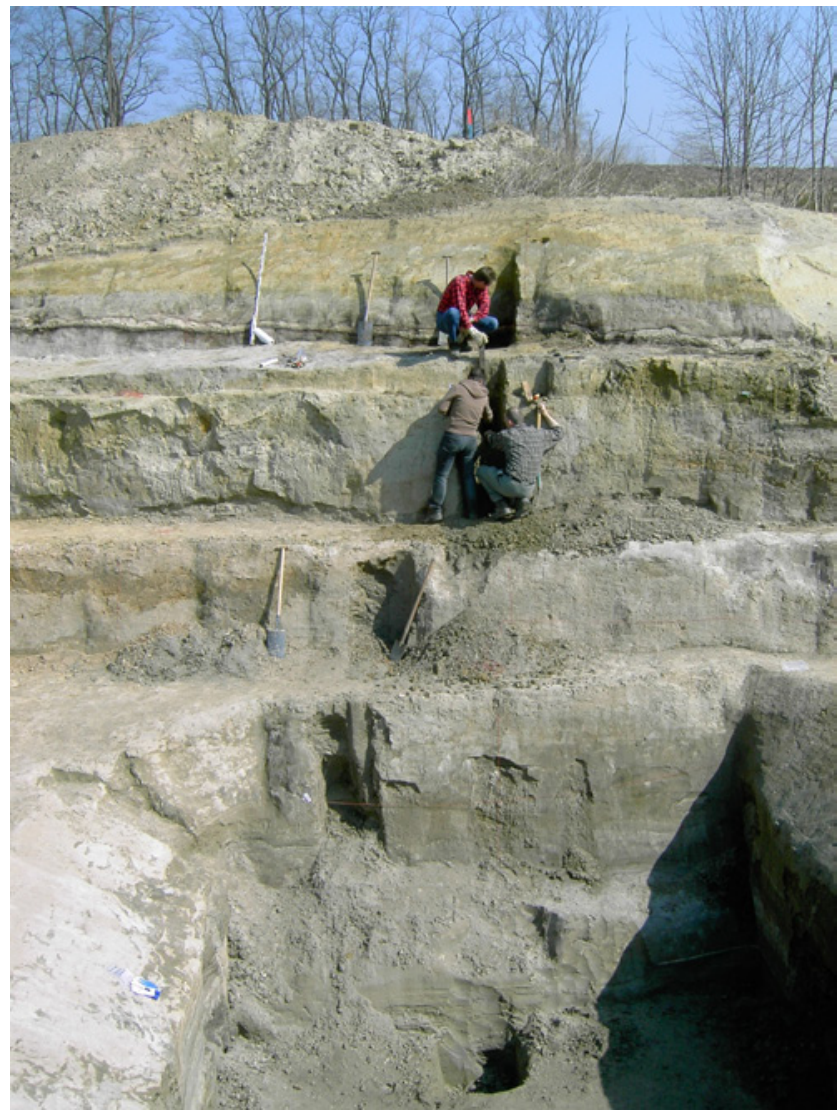

Abb. 7: Hauptprofil A im Becken NN2 (Foto: S. WANSA, 28.3.2007).

Fig. 7: Main profile A of basin NN2 (photo: S. WANSA, 28 March 2007).

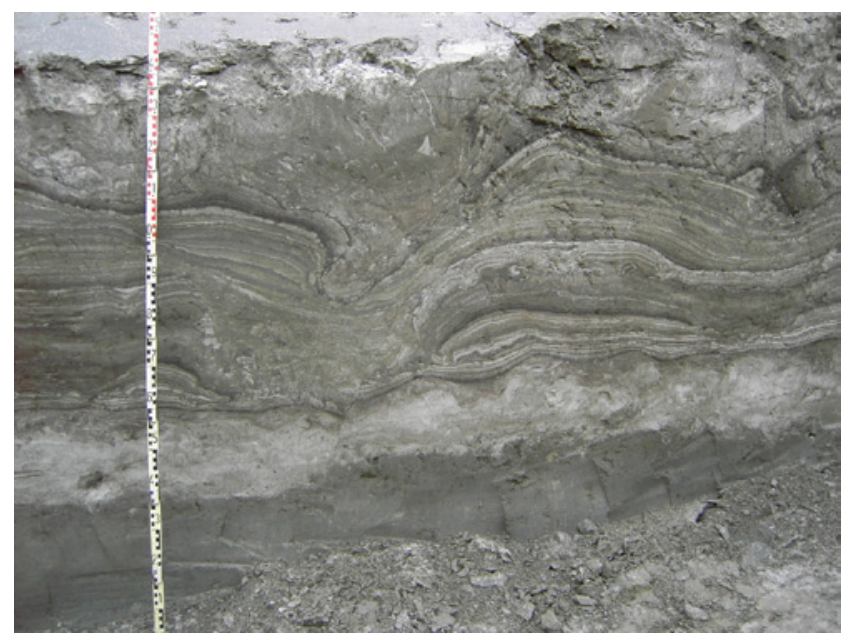

Abb. 8: Schichtdeformationen bei ca. 95,8 bis 96,8 $\mathrm{m}$ NN nahe am Hauptprofil A (Foto: S. WANSA, 20.12.2006).

Fig. 8: Deformed bedding between about 95,8 and 96,8 $\mathrm{m}$ a.s.l. near main profile A (photo: S. WANSA, 20 December 2006).

(mit weniger als $5 \%$ Sand), der im frischen Zustand graublau, sonst braungrau erscheint (Pr. 1 und 2). Vereinzelt wurden hier tonige Kalkkonkretionen beobachtet (Tab. 1: Pr. Neu 2a). Zum Hangenden tritt der blaue Farbton bis auf einzelne Lagen zurück und die Schichtung wird durch helle Feinsandlaminen deutlicher (Pr. 3). Darüber folgt ein recht heterogener Profilabschnitt, der durch die Proben 4-9 repräsentiert wird. Um die lithologische Bandbreite der an der Profilwand aufgeschlossenen Sedimente zu erfassen, sind die Proben 4-6 bis $1,5 \mathrm{~m}$ lateral versetzt entnommen worden. Probe 4 re- präsentiert dunkelbraungrauen schwach tonigen Schluff mit olivgrauen Schlieren, Probe 5 weißgrauen schwach sandigen Grobschluff mit dunkleren, feinkörnigeren Lagen. Die dunklen, braungrauen bis graubraunen Seeschluffe mit Tongehalten um $10 \%$ der Proben 6-9 sind durch hellere Grobschluff- und Feinsandlagen und -linsen geschichtet. Den Bereich von Probe 6 charakterisiert eine deutlich ausgeprägte Feinschichtung. Darüber wird die Schichtung zunächst etwas gröber, wobei die helleren Lagen zurücktreten (Pr. 7 und 8), und nachfolgend undeutlicher (Pr. 9). Der gesamte Profilabschnitt der Proben 5-9 (ca. 95,8-97,2 m NN) weist markante Schichtdeformationen auf (Abb. 8), die als synsedimentäre Fließgefüge interpretiert werden können. Im höheren Teil waren einzelne diapirartige Verwürgungen zu beobachten.

Im lateralen Abstand von wenigstens einigen Dezimetern sind bis in den Bereich der Proben 9 und 10 (vereinzelt auch noch höher) Abschiebungen mit Versatzbeträgen von meist nur wenigen Zentimetern festgestellt worden. Als Ursache für die Zerrungsstrukturen kommt vor allem eine differenzierte Setzung durch Kompaktion im unteren Teil der Beckenfüllung in Betracht. Vereinzelt treten kleine keilförmige Rissbildungen auf, die aber nicht die für Eiskeile typische keilwandparallele Füllung aufweisen. Auch andere, auf Permafrost hindeutende Schichtdeformationen - wie von LAURAT et al. (2007) beschrieben - wurden nicht nachgewiesen und sind nach den paläontologischen Befunden (4.3.3 und 4.3.4, Abb. 6) auch nicht zu erwarten. Der Profilabschnitt der Proben 5-9 entspricht dem archäologischen Fundhorizont 2/2 nach LAURAT \& BRÜHL (2006) und LAURAT et al. (2006) .

Mit Probe 10 beginnt ein weitgehend ungestörter und deutlich feinkörnigerer Profilabschnitt. Die überwiegend graubraunen Schluffe (Proben 10-15) weisen Tonanteile von $18 \%$ bis $38 \%$ auf, die bis Probe 14 stetig zunehmen. Die Sandgehalte liegen meist unter $5 \%$. Zum Hangenden erscheint der Seeschluff überwiegend massig, nur einzelne kleine Feinsandlinsen und -schlieren deuten auf eine schwache Schichtung hin. Eisenoxidausfällungen im Bereich von Probe 12 wurden von LAURAT et al. (2007) aufgrund des Polygonalgefüges als Nassbodenbildung interpretiert. Unmittelbar darüber treten horizontbeständig helle schluffige Feinsandlinsen mit einzelnen Kiesen auf, die zusammen mit dem auflagernden Schluff (Pr. 13) den archäologischen Fundhorizont NN2/1c bilden (Abb. 6, LAURAT \& BRÜHL 2006, LAURAT et al. 2007). Im Niveau der Proben 11 und 12 wurden bis ca. $5 \mathrm{~cm}$ große Kalkkonkretionen registriert (Tab. 1: Pr. Neu 6a). Aufwärts ab dem Bereich von Probe 14 kommen vereinzelt Gipsausblühungen vor.

Bei ca. 99,5 m NN folgt im Hangenden graubrauner, teils olivgrauer toniger Schluff, der durch starke Gipsausblühungen mit Kristallbildung sowie durch Eisenoxidfällungen (z. T. auf Trennflächen und an fossilen Wurzelröhren) gekennzeichnet ist (Pr. 16). Im oberen Abschnitt (Pr. 17) ist er durchgehend oxidiert.

Darüber schließt ein bereits visuell durch starke Farbunterschiede auffällig gegliederter Abschnitt mit zwei dunklen, organisch geprägten Straten und einer hellen Kalkmudde-

3 Die archäologischen Fundhorizonte $2 / 0$ und 2/2 sowie der Fundkomplex 2/1 im Sinne von LAURAT \& BRÜHL (2006) entsprechen den ebenso bezeichneten Profilabschnitten in MANIA et al. (2008) nur teilweise. 
schicht an (Abb. 9, Pr. 18-20). Der untere, $15 \mathrm{~cm}$ mächtige dunkelbraungraue bis braunschwarze Bereich (Pr. 18) besteht zum größeren Teil aus Schluffmudde, die im basalen Teil noch einzelne Gipsausblühungen aufweist. Zum Hangenden sind zunehmend Pflanzenreste enthalten, die den Übergang $\mathrm{zu}$ ca. $5 \mathrm{~cm}$ Detritusmudde ${ }^{4}$ einleiten. Stellenweise wurden hier Vivianit-Ausfällungen beobachtet. Nach einer geringmächtigen dunkelgrauen Schluffmuddeschicht folgen 4-5 cm Kalkmudde (Pr. 19), die aufgrund ihrer hell- bis weißgrauen Farbe im Gelände als Seekreide angesprochen wurde. Sie ist voller Molluskenschalen und enthält auch Pflanzenreste. Den nur $2 \mathrm{~cm}$ mächtigen oberen organischen Bereich (Pr. 20) bildet dunkelbraungraue Algenmudde.

Die auffällige „Schwarz-Weiß-Schwarz“-Sequenz zeigt eine leicht wellige Lagerung und fällt sehr flach nach S bis SE ein (Abb. 7 in Höhe Standplatz der oberen Person). Im ca. $10 \mathrm{~m}$ südlich vom Hauptprofil A gelegenen Profil B befindet sich die Abfolge jedoch bereits wieder in einem etwas höheren Niveau als im Hauptprofil A (Abb. 6). Die Mächtigkeiten und die lithologische Ausbildung der einzelnen Schichtglieder differieren zwischen beiden Profilen nur geringfügig.

Über den farblich so markanten Schichten lagert braungrauer stark toniger Schluff (Pr. 21 und 22), der aufgrund einer Vielzahl von Molluskenresten im Gelände als Kalkmudde angesprochen wurde. Er geht in oxidierten, ockerbraunen tonig-sandigen Schluff über (Pr. 23 und 24a), der ebenfalls noch zahlreiche Schalenreste aufweist. Zum Hangenden treten zunehmend sandigere Abschnitte auf, insbesondere verbraunte, olivgraue bis braunolive, stark schluffige Sande mit gröberen Sandschlieren (Pr. 24b und 25). Es handelt sich hier um den Verzahnungsbereich von limnischen Bildungen mit schwemmlössartiger Fließerde, der den oberen Abschluss der Beckenfüllung (und des Hauptprofils A) bildet.

Nach den Analysenergebnissen beträgt der Kalkgehalt der Grundmoräne 5-6\%. Eine Kalkreduzierung im Hangendbereich des Geschiebemergels ist demnach, wie auch in Profil D, nicht festzustellen. Die meisten anderen Proben aus der Rammkernsondierung enthalten 5-9 \% Kalk, nur in den Pr. US 1 und US 6 werden mit 12,9 \% bzw. 11,7 \% höhere Werte (Abb. 6) erreicht. Im unteren und mittleren Teil des Schurfprofils (Pr. 1-15) liegen die Werte recht stabil bei $10-12 \%$. Mit den einsetzenden Gipsausfällungen und der Oxidation geht eine starke Kalkreduzierung bis hin zur völligen Entkalkung einher (Pr. 16 und 17), auch die Probe 18 aus der Schluffmudde/Detritusmudde ist kalkfrei. Darüber nehmen die Kalkgehalte deutlich zu, doch nicht in dem Maße, wie die Feldansprache annehmen ließ. Probe 19 umfasst neben Kalkmudde vermutlich auch Material aus der dünnen Schluffmuddeschicht, die direkt darunter lagert, woraus der relativ geringe Kalkgehalt (5,3\%) resultieren dürfte. Die Analyse der 2003 in Profil B1 aus der Kalkmudde entnommenen Probe B1/17 ergab $44 \% \mathrm{CaCO}_{3}$. Die Algenmudde (Pr. 20) führt $10 \%$ Kalk, und in der hangenden, stark Molluskenschalen führenden Kalkmudde (Pr. 21 und 22) wurden 16-18\% ermittelt, was sich mit der Analyse aus dem gleichen Horizont in Profil B1 deckt. Im obersten Profilbereich (Pr. 23-25) sind in Abhängigkeit vom Sandgehalt

4 Die Feldansprache als „Algenmudde“ hat sich bei der Pollenanalyse nicht bestätigt, da die massenhafte Ausbreitung der Algen erst am oberen Ende dieser Schicht einsetzte.

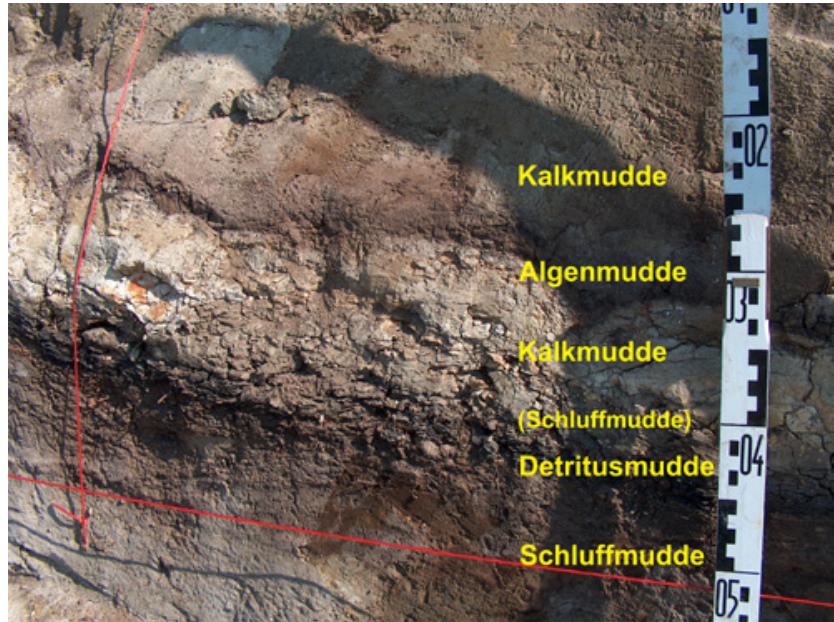

Abb. 9: Helle Kalkmudde zwischen Detritusmudde und Algenmudde im Hauptprofil A (Foto: N. HERMSDORF, 28.3.2007).

Fig. 9: Light calcareous mud between organic muds in main profile A (photo: N. HERMSDORF, 28 march 2007).

8-12\% Kalk enthalten. Schließlich seien die beiden aus verschiedenen Niveaus stammenden schaligen tonigen Kalkkonkretionen erwähnt: Probe 2a (aus dem tiefsten Teil des Aufschlusses) besteht zu $21 \%$ aus Calcit, Probe 6a aus dem Niveau von $98 \mathrm{~m} \mathrm{NN}$ zu $60 \%$.

Die Anteile an organischer Substanz sind im Allgemeinen gering (Abb. 6). An Material aus der Rammkernsondierung wurde in der Regel weniger als $2 \%$ festgestellt. Etwas höhere und bereits bei der Kernansprache erkannte Gehalte führen die Proben US 7, US 10 und US 11 (3,7-4,5\%). Sie sind, auch in Anbetracht der dahingehenden palynologischen Ergebnisse (4.3.3.1), wahrscheinlich auf die Umlagerung kohligen Materials zurückzuführen. Im Schurfprofil liegen die Humusgehalte meist unter $1 \%$. Werte von 1-2 \% sind in den Proben 4, 6, 17, 21 und 22 ermittelt worden. Lediglich die Schluffmudde/Detritusmudde-Strate sowie die Algenmudde erreichen Humusgehalte von 11-13 \% (Pr. 18, Pr. B1/16) und 6-9 \% (Pr. 20, Pr. B1/18), was für organische Mudden $\mathrm{zu}$ gering ist (vgl. AD-HOC-ArBeITSGRUPpe Boden 2005, Hinze et al. 1989, Merkt et al. 1971). Auch die als Kalkmudde bezeichneten Sedimente enthalten $\mathrm{zu}$ wenig bzw. kaum nachweisbare organische Substanz und müssten daher korrekt als „kalkiger Schluff“ bezeichnet werden. Wir geben hier jedoch der Feldansprache den Vorrang, zumal nicht ausgeschlossen werden kann, dass bei der Beprobung der z. T. sehr dünnen Lagen auch Fremdmaterial erfasst wurde.

\subsubsection{Mineralogie und Geochemie [J. Luckert]}

Die Ergebnisse der quantitativen Röntgenphasenanalyse zeigen, dass in allen (Gesamt-)Proben Quarz, Feldspäte, Karbonate und Tonminerale die Hauptkomponenten bilden (Tab. 1). Bei den Tonmineralen dominieren Illit und unregelmäßige Illit-Smektit-mixed-layer-Minerale mit Smektitschichtanteilen von 40-55\%, außerdem kommen stets Kaolinit und in geringerer Menge auch Chlorit vor. Weiterhin konnten gelegentlich und meist nur in Spuren Pyrit und Amphibol festgestellt werden, auch Anteile von amorphen Phasen wurden indirekt nachgewiesen. 
Die Gehalte an Quarz variieren aufgrund der überwiegend schluffigen Beschaffenheit der Proben im Allgemeinen zwischen 40 und 55 Masse- $\%$, nur in den untersten tonreicheren Basisproben (U 21, U 19 und U 17) liegen die Quarzgehalte unter 30 Masse-\%. Betrachtet man die ersten drei Meter des Profils mit den Proben U 21-U 10, wo relativ große Sandgehalte auftreten (4.3.1: Proben US 11-US 9), so ist eine kontinuierliche Quarzzunahme von 27 bis hin zu 55 Masse-\% erkennbar. Auch in den Texturpräparaten der Tonfraktion $<2 \mu \mathrm{m}$ war Quarz in geringer Menge stets nachweisbar.

An Feldspäten konnten sowohl Kalifeldspat als auch albitreicher Plagioklas festgestellt werden; sie treten immer zusammen in annähernd gleich großen Anteilen auf. In den tonreicheren Proben U 21-U 13 sind parallel zum Quarzgehalt auch die Gehalte an Feldspäten signifikant niedriger (Tab. 1). In den Texturaufnahmen der Tonfraktion $<2 \mu \mathrm{m}$ waren beide Feldspäte ebenfalls in Spuren vorhanden.

An Karbonaten kommen sowohl Calcit als auch Dolomit vor, wobei die Gehalte an Calcit deutlich höher sind. Röntgenographisch sind beide anhand ihrer unterschiedlichen Beugungsspektren eindeutig voneinander zu unterscheiden. Während die Gehalte an Dolomit bei 1-3 Masse-\% liegen, variieren die Gehalte an Calcit im Allgemeinen zwischen 5 und 10 Masse-\%. Abgesehen von den beiden Karbonatkonkretionen Neu 2a bzw. 6a und den nicht beprobten Seekreidelagen sind erhöhte Calcitgehalte oft auf eine Anreicherung von Muschelschill (z. B. Pr. Neu 12, U 1 und U 2) zurückzuführen.

Amphibol und Pyrit kommen nur vereinzelt und in Spuren vor. Pyrit kann als Beleg für zeitweise synsedimentäre reduzierende Milieuverhältnisse angesehen werden, vorausgesetzt, es handelt sich nicht um Umlagerung. Für reduzierte Bedingungen sprechen auch die weitestgehend fehlenden Kleinmuscheln und die geringen Arten- und Individuendichten bei Süßwasserschnecken (4.3.4).

In einigen Proben konnten auch amorphe Phasen indirekt als defizitärer Rest zu $100 \%$ kristalliner Phase abgeleitet werden. Nach makroskopischer Bewertung der Sandfraktion ist der Anteil an organischer Substanz $\left(\mathrm{C}_{\text {org }}\right)$ dabei nur gering und liegt nach CNS-Analysen an anderen vergleichbaren (Gesamt-)Proben bei $\leq 1 \%$. Der überwiegende Teil ist anorganischer Natur und enthält vor allem $\mathrm{Ca}$ - und Fe-Anteile, untergeordnet auch $\mathrm{Al}, \mathrm{Mg}$ und $\mathrm{Si}$. Um welche amorphen Verbindungen es sich in diesen Proben handelt, lässt sich daraus allerdings nicht ableiten.

$\mathrm{Zu}$ den Tonmineralen, die in allen Proben vorkommen, gehören Illit, Illit-Smektit-Wechsellagerungsminerale, Kaolinit und Chlorit. Als häufigstes Tonmineral tritt ein Illit-Smektit-mixed-layer-Mineral (nachfolgend ml-Mineral genannt) mit Smektitschichtanteilen von 40-55\% auf. Aufgrund der charakteristischen Veränderungen von Peaklage, -form und -intensität handelt es sich ausschließlich um unregelmäßig geordnete ml-Minerale vom Typ R0 nach Moore \& Reynolds (1997). Andere Illit-Smektit-ml-Minerale (regelmäßige oder partiell geordnete vom Typ R1 und R3) oder sogar Smektite konnten in keiner Probe nachgewiesen werden. Die Herkunft dieser ml-Minerale ist in Norddeutschland an die weite Verbreitung mächtiger mariner Tertiärtone gebunden, in denen sie die tonmineralogische Hauptkomponente bilden. Bei jedem Inlandeisvorstoß wurden Teile dieser Tertiärtone aufgearbeitet und sind be- sonders in den Geschiebemergeln und Bändertonen sowie deren Abtragungsprodukten anzutreffen.

In den untersuchten Proben ist Illit nach den ml-Mineralen das zweithäufigste Tonmineral. Da bis auf die beiden Konkretionen (Pr. Neu 2a und 6a) von jeder Probe die Tonfraktion $<2 \mu \mathrm{m}$ für die Herstellung von Texturpräparaten gewonnen wurde, konnte eine separate Aushaltung von Illit und ml-Mineralen vorgenommen werden. In vielen Proben sind auch Spuren von Muskovit vorhanden, der makroskopisch in der abgetrennten Sandfraktion als Hellglimmerschüppchen zu beobachten war.

In allen Proben kommen geringe Mengen an Kaolinit und eines Chlorit-Minerals (im Folgenden kurz Chlorit genannt) vor, nur in den zwei Konkretionen (Pr. Neu 2a und 6a) liegen die Gehalte unterhalb der röntgenographischen Nachweisgrenze. Die Absolutgehalte von Kaolinit/Chlorit sind mit 2-7 Masse-\% relativ niedrig, wobei die Werte von Kaolinit stets höher sind als die von Chlorit (Tab. 1). Die höchsten Kaolinitgehalte wurden im basalen Bereich des Profils festgestellt, zum Hangenden hin ist tendenziell eine Abnahme erkennbar. Bei dem hier vorkommenden trioktaedrischen Chloritmineral dürfte es sich nur um primären Chlorit als Abtragungsprodukt einer vorwiegend physikalischen Verwitterung handeln. Hinweise auf Anteile von sekundärem, durch Pedogenese neu gebildetem Bodenchlorit sind nicht vorhanden. Auch weitere typische, durch Pedogenese neu gebildete Tonminerale, wie z. B. Chlorit-Vermiculit-mixedlayer-Minerale oder Vermiculit, konnten in keiner Probe festgestellt werden. Allerdings sind die Proben-Intervalle zu groß, um entsprechende Bodenbildungen im Profil sicher ausschließen zu können.

Die mineralogische und geochemische Zusammensetzung der Proben ist, abgesehen von einer Zweiteilung im unteren Profilabschnitt, relativ gleichförmig. So weisen die untersten 1,5-2 m des Profils (Pr. U 21-U 15) mit 44-56\% einen deutlich höheren Gehalt an Tonmineralen auf als die darüber liegenden Schichten mit nur 20-34 \%. Die hohen Gehalte an Tonmineralen, insbesondere von Illit/Muskovit und den ml-Mineralen, korrelieren mit erhöhten Werten von Aluminium, Rubidium und Vanadium, da sie Bestandteile der kristallinen Zusammensetzung dieser Dreischichtsilikate sind. Weiterhin ist gegenüber den darüber liegenden Sedimenten zu beobachten, dass sowohl die Gehalte an Kaolinit als auch die Smektitschichtanteile der ml-Minerale in dieser basalen Abfolge etwas höher sind.

Korrespondierend mit den hohen Anteilen der Tonfraktion und trotz der ebenfalls hohen Sandkomponenten sind die Quarzgehalte in den untersten Schichten der Beckenfüllung besonders gering. Geochemisch zeigt sich das in niedrigeren $\mathrm{SiO}_{2}$ - und Zirkonwerten. Parallel zu den niedrigen Quarzgehalten sind in diesen Proben auch die Gehalte an Feldspäten signifikant niedriger als in den nachfolgenden Profilabschnitten.

\subsubsection{Palynologie [J. Strahl]}

Die palynologischen Untersuchungen dienten in erster Linie der relativen zeitlichen Einstufung der Sedimentfüllung des Beckens NN2. Des Weiteren wurde versucht, eine abschließende Klärung der seit ca. 20 Jahren umstrittenen stratigraphischen Position der benachbart liegenden 
Tab. 1: Ergebnisse der quantitativen Röntgenphasenanalyse von Proben aus dem Becken NN2, Hauptprofil A (Angaben in Masse-\%). ml *-Illit-Smektitmixed-layer-Minerale mit Smektitschichtanteilen von 40-55\%.

Tab. 1: Results of quantitative X-ray phase analysis of samples from basin NN2, main profile A (figures in mass \%). ml *-illite-smectite-mixed-layerminerals with 40-50\% smectite layers.

\begin{tabular}{|c|c|c|c|c|c|c|c|c|c|c|c|c|}
\hline Probe & Quarz & $\begin{array}{l}\text { Kalifeld- } \\
\text { spat }\end{array}$ & $\begin{array}{l}\text { Plagio- } \\
\text { klas }\end{array}$ & Calcit & Dolomit & Pyrit & $\underset{\text { bol }}{\text { Amphi- }}$ & Kaolinit & Chlorit & Illit & ml* & $\begin{array}{c}\text { Amorphe } \\
\text { Phase }\end{array}$ \\
\hline Neu 13 & 54 & 5 & 6 & 8,5 & 1 & - & - & 1 & 0,5 & 10,5 & 13,5 & - \\
\hline Neu 12 & 35 & 5 & 4 & 16 & 1 & 1,5 & - & 2,5 & 0,5 & 14 & 17 & 3,5 \\
\hline Neu 11 & 56 & 7 & 5 & 0,5 & 0,5 & - & - & 1,5 & 0,5 & 17 & 12 & - \\
\hline Neu 10 & 46 & 5 & 6 & 5 & 3 & 1 & - & 2 & 0,5 & 11,5 & 15 & 5 \\
\hline Neu 9 & 42 & 5 & 5 & 8,5 & 1,5 & - & - & 2 & 1 & 14 & 16 & 5 \\
\hline Neu 8 & 50 & 6 & 5 & 8 & 2 & - & - & 2 & 1 & 13 & 13 & - \\
\hline Neu 7 & 47 & 5 & 6 & 9 & 3 & - & - & 2 & 1 & 11,5 & 15,5 & - \\
\hline Neu 6a & 10 & 2 & - & 60 & 0,5 & - & - & - & - & 9 & - & 18,5 \\
\hline Neu 6 & 45 & 6 & 6 & 10 & 3 & - & - & 2 & 1 & 13 & 14 & - \\
\hline Neu 5 & 48 & 7 & 6 & 5 & 3 & - & - & 2 & 1 & 13,5 & 11,5 & 3 \\
\hline Neu 4 & 50 & 9 & 7 & 8 & 2,5 & - & 1 & 1,5 & 1 & 11 & 9 & - \\
\hline U 1 & 47 & 5,5 & 5 & 11 & 2 & 0,5 & - & 2 & 1 & 9 & 13 & 4 \\
\hline Neu 3 & 51 & 6 & 5 & 7,5 & 2 & 2 & - & 1,5 & 0,5 & 9 & 11 & 4,5 \\
\hline U 2 & 43 & 5 & 6 & 11 & 2 & 1 & 1 & 2,5 & 1,5 & 11 & 14 & 2 \\
\hline Neu $2 a$ & 36 & 4 & 3,5 & 21 & 1,5 & - & - & - & - & 12 & - & 22 \\
\hline Neu 2 & 56 & 5 & 6 & 7,5 & 2 & - & - & 1,5 & 0,5 & 7 & 13 & 1,5 \\
\hline U 4 & 53 & 5 & 6 & 7,5 & 2,5 & 1 & - & 2 & 1 & 9 & 13 & - \\
\hline Neu 1 & 54 & 4,5 & 6 & 8 & 2,5 & 1 & 1 & 2 & 1 & 6 & 14 & - \\
\hline U 6 & 44 & 7 & 5 & 4 & 2,5 & 0,5 & 1 & 3 & 1 & 12 & 16 & 4 \\
\hline U 8 & 57 & 6 & 8 & 6 & 3 & - & - & 1,5 & 0,5 & 7 & 11 & - \\
\hline U 10 & 55 & 7 & 6 & 7 & 2,5 & 1 & - & 1,5 & 1 & 10 & 9 & - \\
\hline U 13 & 45 & 4 & 4 & 6 & 2 & 1 & - & 2,5 & 1,5 & 13 & 15 & 6 \\
\hline U 15 & 39 & 5 & 3,5 & 7 & 1,5 & - & - & 4 & 2 & 19 & 19 & - \\
\hline U 17 & 29 & 2,5 & 4 & 8,5 & 1 & - & - & 5 & 2 & 22 & 26 & - \\
\hline U 19 & 28 & 2 & 3 & 8,5 & 1,5 & - & 1 & 5 & 2 & 23 & 26 & - \\
\hline U 21 & 27 & 2,5 & 3 & 9 & 1,5 & - & - & 5 & 2 & 31 & 14 & 5 \\
\hline
\end{tabular}

Becken NN1 und NN2 herbeizuführen. Hier steht nach wie vor die intrasaalezeitliche Einstufung von NN1 durch zuletzt MANIA et al. (2010), ausschließlich diskutiert anhand der palynologischen Untersuchungen von SEIFERT (1990) bzw. SEIFERT-Eulen (2010), einer eemzeitlichen von NN1 (Litt 1994a, b, Kremenetski in Boettger et al. 2005, 2007) und NN2 (Kremenetski in Mania et al. 2008, 2010, KREMENETSKI 2010) gegenüber. Darüber hinaus existiert ein wesentlicher Widerspruch in der unterschiedlichen altersmäßigen Deutung des archäologischen Hauptfundhorizontes NN2/2 an sich und des daraus resultierenden stratigraphischen Gesamtstatus des Beckens NN2. So ist die Positionierung des Fundhorizontes NN2/2 unter die von KREMENETSKI pollenanalytisch untersuchte Sequenz (LAURAT et al. 2006: 88, MANiA et al. 2008: 20, MANiA et al. 2010) problematisch, da dies hinsichtlich des bis dahin palynologisch nicht stratifizierten Fundhorizontes zur Ausweisung einer weiteren, ebenfalls intrasaalezeitlichen Warmzeit führte. Zum anderen wird der stark mit Umlagerungen behaftete Teil des darüber folgenden Pollenprofils (Lokale Pollenzonen LPZ 1-2) einer als warthezeitlich aufgefassten Kaltphase zugeordnet, über welcher erst dann die Ablagerungen der jüngeren Eem-Warmzeit (LPZ 3-8) folgen sollen. $\mathrm{Zu}$ bemerken ist an dieser Stelle, dass das Pollenprofil von KREMENETSKI aus einem Beckenbereich stammt, in dem der Fundhorizont NN2/2 nicht aufgeschlossen war (T. LAURAT, freundl. mündl. Mitt.). Daraus ergäbe sich für NN2 unter Betrachtung des durch MANIA ausgewiesenen konstruierten Gesamtprofils eine Abfolge von mehreren Kalt- und Warmphasen, die den Zeitraum vom DrentheStadium des Saale-Komplexes bis in das Holozän umfassen würde (zuletzt MANiA et al. 2010: Abb. 42). Die aktuellen Befunde (Abb. 10) belegen jedoch, dass die archäologischen Fundhorizonte NN2/2 und NN2/1c innerhalb der EemWarmzeit liegen.

Die Darstellung der aus 122 untersuchten Proben des Hauptprofils A ermittelten Pollenspektren erfolgte in einem prozentualen Pollendiagramm (Abb. 10 und 11). Proben, die sich als pollenleer bzw. extrem pollenarm erwiesen, blieben dabei unberücksichtigt. Die zur Gruppe der Gehölze (BP) gehörigen Taxa wurden nach ihrer Einwanderungsfolge in das Untersuchungsgebiet farblich abgesetzt angeordnet, lediglich Kiefer (Pinus) und Birke (Betula) sind separat in Verbindung mit der Gesamtsumme der BP und der Gesamtsumme der Kräuter (NBP) abgebildet. Diese Art der Darstellung wurde zum Zwecke des besseren Vergleichs, hier allerdings im Wesentlichen auf die Abbildung der BP-Kurven beschränkt, auch auf die Pollendiagramme von Seifert (1990), Litt (1994a, b) und Kremenetski (in Boettger et al. 2005, 2007) für das Becken NN1 angewandt (vgl. Abb. 11b-d). Auf eine dementsprechend veränderte Abbildung des Diagramms von Kremenetski (2010: Abb. 1) für NN2 musste aus folgenden Gründen verzichtet werden: Zum einen beinhaltet die Tab. 2 (S. 278 ff.) lediglich die je Probe ermittelten Pollenkonzentrationen, deren Ableitung im Text nicht formuliert ist, nicht aber die tatsäch- 
lichen Zähl- bzw. Prozentwerte. Eine zweifelsfreie Neuberechnung war dadurch nicht möglich. Zum anderen schloss sich auch eine einfache Umzeichnung des Diagramms wegen der offensichtlichen Einbeziehung eindeutig tertiärer Taxa (konkret Myrica und Castanea) in die statistische Berechnungssumme aus.

Das Pollendiagramm für das Hauptprofil A (Abb. 10) beschränkt sich neben den BP auf ausgewählte, besonders häufige Taxa. Seltenere Formen finden z. T. im Text verbale Erwähnung. Die bei der Darstellung der ermittelten und nachfolgend diskutierten saalespätglazialen bis frühweichselzeitlichen Vegetationsentwicklung angeführten Prozentwerte reflektieren ausschließlich Pollenfrequenzen und sind nicht mit der etwaigen Häufigkeit eines Taxons am Untersuchungspunkt bzw. dessen Umgebung gleichzusetzen. Alle Kurven im Pollendiagramm sind zehnfach überhöht abgebildet, um auch Werte unter $1 \%$ darstellen zu können. Palynomorphen, die nach Erreichen der erforderlichen Grundsumme im Präparat vorgefunden wurden, sind mit einem + im Diagramm aufgeführt. Um die Ergebnisse zu den im Bereich der Profilschiene 6 aus den Detritus- und Kalkmudden im Abstand von $1 \mathrm{~cm}$ entnommenen Proben (Abb. 4) zeichnerisch darstellen zu können, wurden die Probenabstände im Pollendiagramm (Abb. 10 und 11a) für diesen Abschnitt verdoppelt. Die PZ 7 erscheint dementsprechend gegenüber der Profildarstellung in Abb. 6 gestreckt!

Die pollenanalytische Gliederung aller nachfolgend abgebildeten Pollendiagramme (Abb. 10 und 11a-d) wurde aus Vergleichsgründen vereinheitlicht. So fanden für das Saale-
Spätglazial die von Strahl \& Hermsdorf (2008) und für die Eem-Warmzeit die von ERD (1973) publizierten und durch die Staatlichen Geologischen Dienste der Länder SachsenAnhalt und Brandenburg genutzten Gliederungen Anwendung (siehe auch Tab. 2). Die in den entsprechenden Publikationen durch die oben angeführten Autoren ursprünglich verwendeten unterschiedlichen Gliederungen der warmzeitlichen Ablagerungen sind in Tab. 2 zum besseren Verständnis gegenübergestellt.

\subsubsection{Saale-Pleniglazial}

\section{PZ A, Waldlose Zeit}

Für den waldfreien Abschnitt des Saale-Pleniglazials nach dem Eisrückgang lassen sich zwei Unterabschnitte mit den PZ A1 und A2 (Abb. 10) aushalten. Die ausschließlich aus der Rammkernsondierung stammenden Proben des Diagrammabschnitts A1 erwiesen sich als sehr pollenarm bis nahezu pollenleer. Insgesamt zeigt sich eine sehr monotone Zusammensetzung der Pollenflora. In der Gruppe der Gehölze dominiert die Kiefer mit überwiegend Pollenfrequenzen um $80 \%$. Seltener erscheinen Birke und Sanddorn (Hippophaë). Neben Fichte (Picea) und Tanne (Abies) wurden außerdem wärmebeanspruchende Gehölze nachgewiesen, die ebenso wie die Kiefer mit Sicherheit nicht autochthon sind. Dazu gehören vor allem Hainbuche (Carpinus), Erle (Alnus) sowie seltener Hasel (Corylus), Eiche (Quercus), Ulme (Ulmus) und Linde (Tilia). Sie können sowohl aus dem Tertiär, angesichts des Nachweises des Großen Algenfarns (Azolla filiculoides)

Tab. 2: Gliederung des Eem-Interglazials in Berlin-Brandenburg (B-B) bzw. Ostdeutschland (ERD 1973) in Gegenüberstellung zu den in Nordwest- (MENKE \& TYNNI 1984) und Mitteldeutschland (LITT 1994a) Anwendung findenden Gliederungen unter Einbeziehung der allgemeinen Warmzeitgliederung nach LANG (1994) und der anhand von fahresschichten ermittelten Dauer der einzelnen Pollenzonen (PZ) nach MüLLER (1974).

Tab. 2: Sub-divisions of the Eemian interglacial in Berlin-Brandenburg (B-B) respectively East Germany compared with the sub-divisions applied to North-West (MENKE \& TYNNI 1984) and Central Germany (LITT 1994a), taking account of the general warm period divisions according to LANG (1994) and the duration of individual pollen zones (PZ) identified on the basis of annual layers by MÜLLER (1974).

\begin{tabular}{|c|c|c|c|c|c|}
\hline \multirow{3}{*}{$\begin{array}{c}\text { Warmzeitphasen } \\
\text { nach } \\
\text { LANG [1994] }\end{array}$} & \multirow{3}{*}{ Waldzeiten } & \multicolumn{3}{|c|}{ Pollenanalytische Gliederung des Eem-Interglazials nach } & \multirow{3}{*}{$\begin{array}{c}\text { Absolute } \\
\text { Dauer nach } \\
\text { MüLLER }[1974]\end{array}$} \\
\hline & & $\begin{array}{c}\text { MeNKE } \\
\text { \& TYNNI [1984] }\end{array}$ & ERD [1973] & LıтT [1994a] & \\
\hline & & $\begin{array}{c}\text { NW- } \\
\text { Deutschland }\end{array}$ & $\begin{array}{c}\text { B-B/Ost- } \\
\text { deutschland }\end{array}$ & $\begin{array}{c}\text { Elbe-Saale- } \\
\text { Gebiet }\end{array}$ & \\
\hline \multirow{2}{*}{$\begin{array}{c}\text { Phase D } \\
\text { [telokratische Ph.] } \\
\text { Kiefern- bzw. Kiefern- } \\
\text { Fichten-Zone }\end{array}$} & Kiefern-Zeit & VII & 9 & 7 & 2000 a \\
\hline & Kiefern-Fichten-Tannen-Zeit & $\mathrm{VI}$ & 8 & $6 b$ & 2000 a \\
\hline $\begin{array}{c}\text { Phase C } \\
\text { [mesokratische } \\
\text { Phase, 2. Teil] } \\
\text { Hainbuchen-Zone }\end{array}$ & $\begin{array}{l}\text { Hainbuchen-Fichten-Zeit } \\
\text { Hainbuchen-Zeit }\end{array}$ & $\begin{array}{l}\mathrm{Vb} \\
\mathrm{Va}\end{array}$ & $\begin{array}{l}7 \\
6\end{array}$ & 5 & 4000 a \\
\hline \multirow[b]{2}{*}{$\begin{array}{c}\text { Phase B } \\
\text { [mesokratische } \\
\text { Phase, } 1 \text {. Teil] } \\
\text { Eichen-Zone }\end{array}$} & Hasel-Eiben-Linden-Zeit & IVb & 5 & $4 b$ & $\sim 1200 \mathrm{a}$ \\
\hline & Eichenmischwald-Hasel-Zeit & IVa & 4 & $4 a$ & $1200 \mathrm{a}$ \\
\hline $\begin{array}{c}\text { Phase A } \\
\text { [protokratische Ph.] } \\
\text { Birken-Kiefern-Zone }\end{array}$ & Kiefern-Eichenmischwald-Zeit & III & 3 & 3 & $\sim 450 \mathrm{a}$ \\
\hline
\end{tabular}




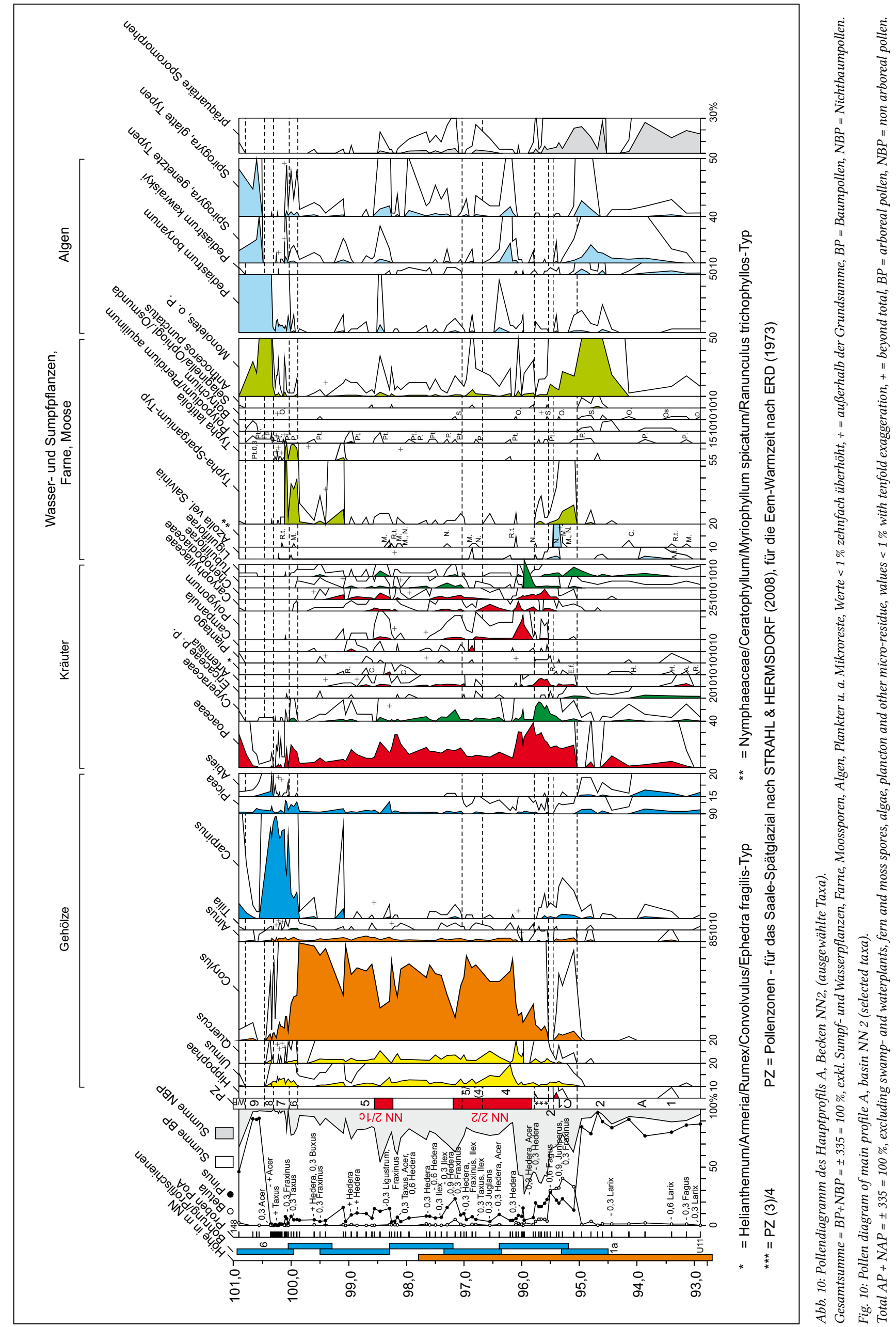




\section{Interglazialbecken NN2}

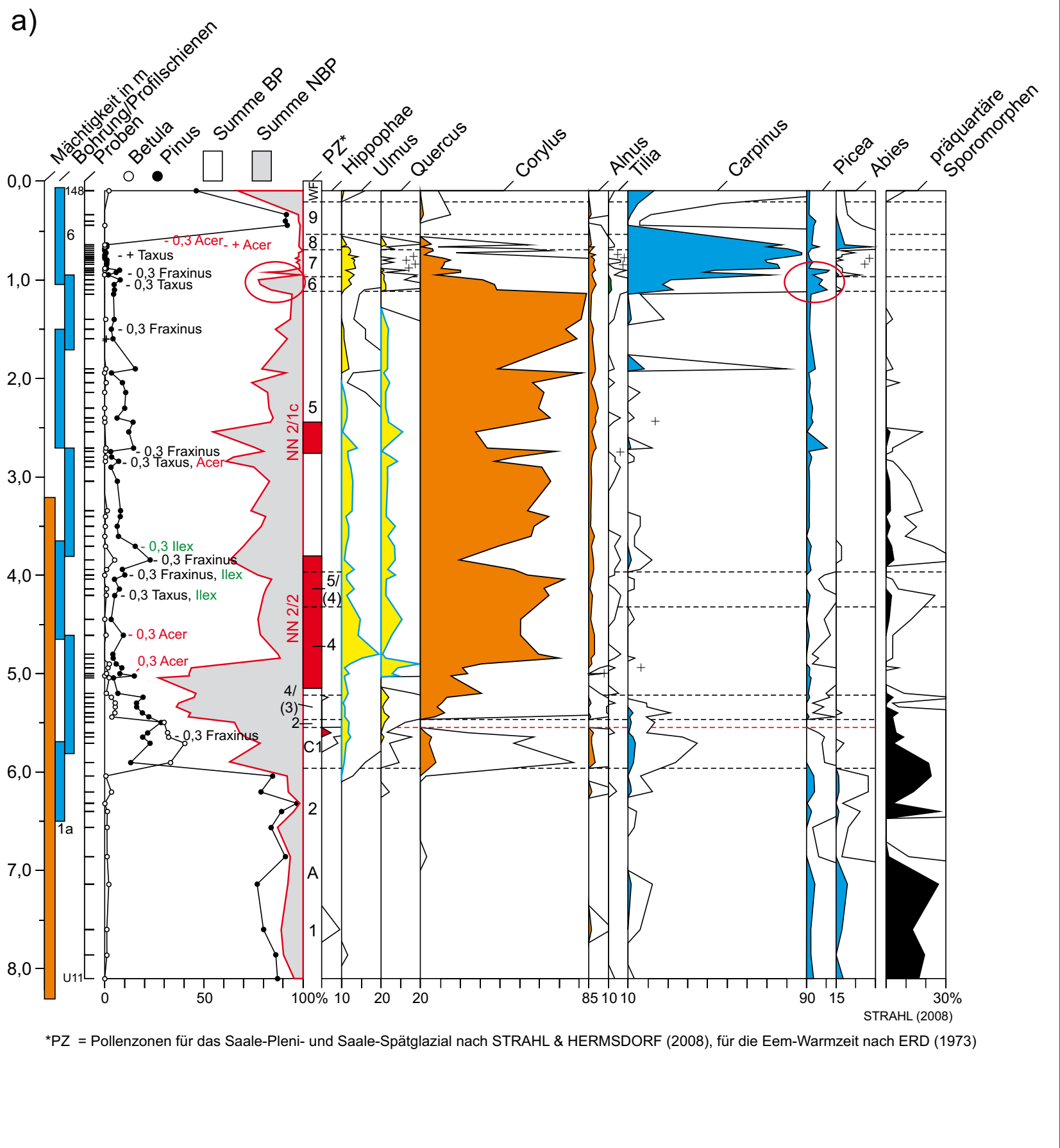

Abb. 11: Gegenüberstellung der aus den Becken NN1 und NN2 pollenanalytisch untersuchten Profile (Darstellung der Gehölzpollenanteile). Fig. 11: Comparison of profiles analysed for pollen and spores from basins NN1 and NN2 (representation of proportions of tree pollen). 


\section{Interglazialbecken NN1}

b)

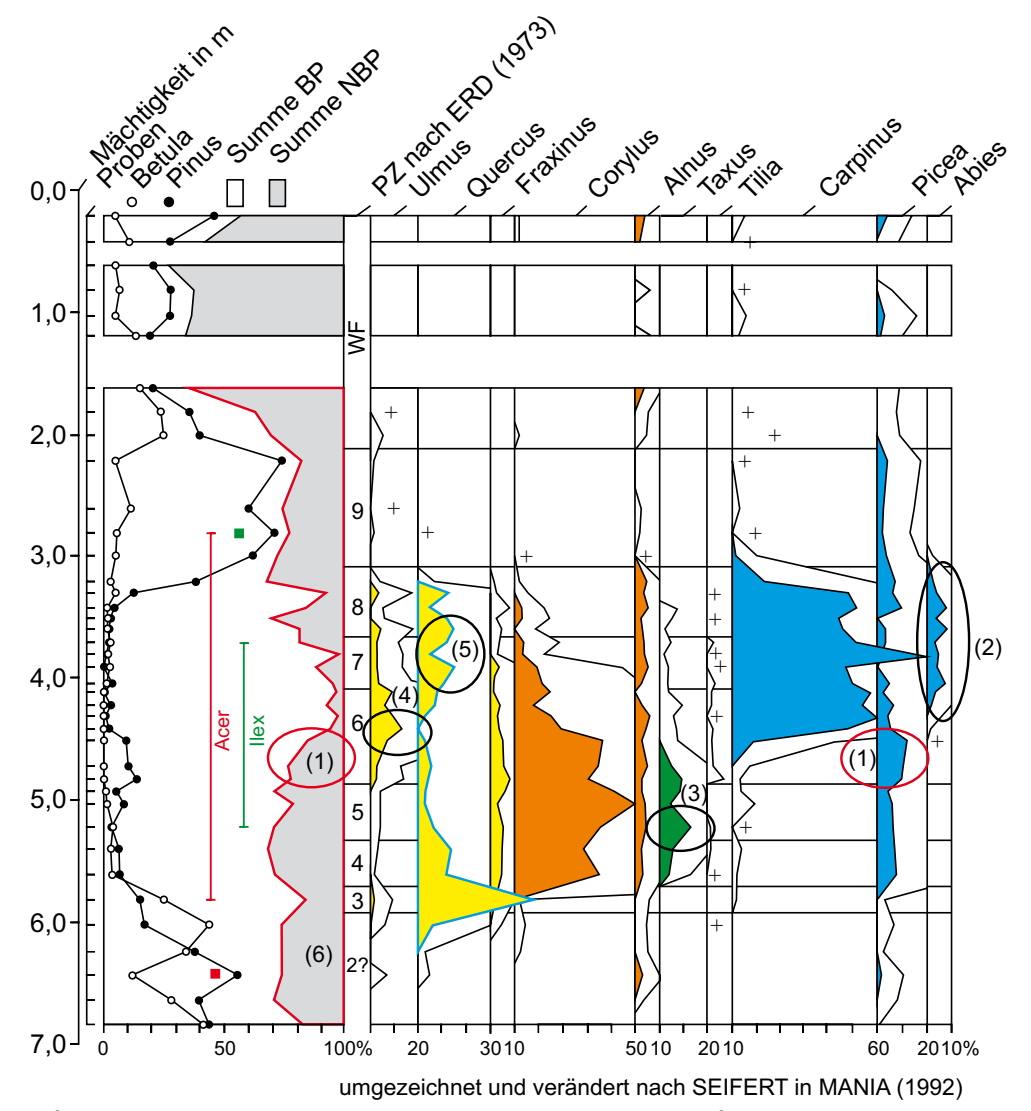

c)

d)

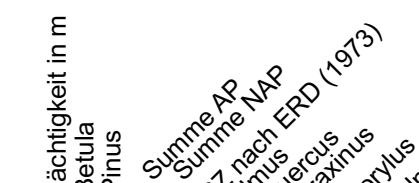

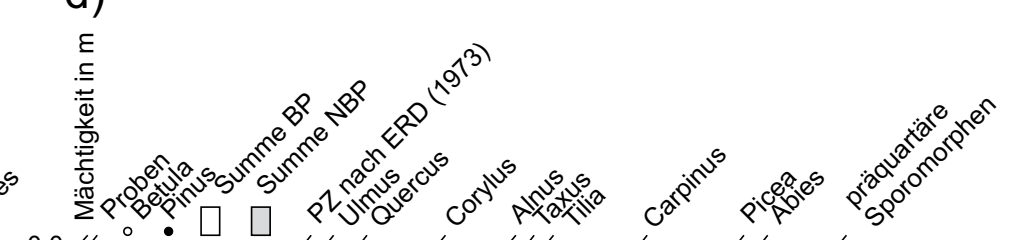
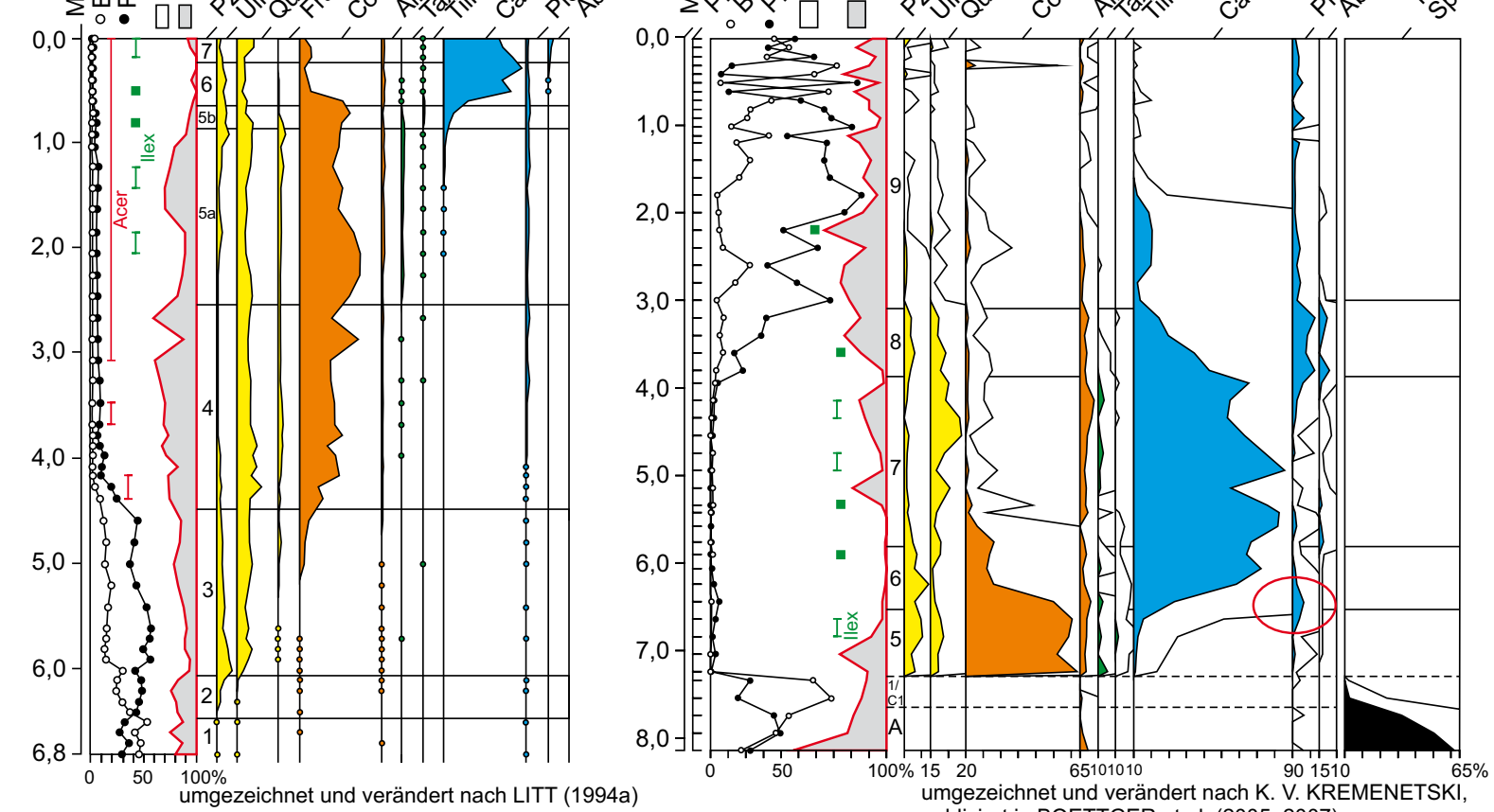

publiziert in BOETTGER et al. $(2005,2007)$ 
aber auch aus einer älteren Warmzeit, beispielsweise aus Altwasserbildungen innerhalb der holstein- bis frühsaalezeitlichen Körbisdorfer Schotter stammen (vgl. 2 und 4.1).

Hervorsticht ein z. T. erheblicher Anteil präquartärer Palynomorphen. Neben Dinozysten sind dies in erster Linie Schirmtanne (Sciadopytis), diverse Tricolporates und trilete Sporen, seltener Amberbaum (Liquidambar), Gagelstrauch (Myrica-Typ), Hickory (Carya), die Gruppe der Sumpfzypressen- und Zypressengewächse (Taxodiaceae/Cupressaceae), Hemlocktanne (Tsuga), Tupelobaum (Nyssa), die zu den Heidekrautartigen (Ericales) zählende Gattung Symplocos, Vertreter der Normapolles-Gruppe u. a. Ein sowohl per Analyse des Humusgehaltes ermittelter als auch makroskopisch über kohlige Partikel festgestellter erster und sich in der PZ A2 nochmals wiederholender Anstieg des organogenen Gehaltes im Sediment (Abb. 6 und 10) geht hier im Wesentlichen auf resedimentierte, präquartäre organogene Ablagerungen zurück.

Eine prozentual gesehen nennenswert entwickelte Offenlandflora existierte zunächst nicht. So finden sich abgesehen von häufigeren Süß- und Sauergräsern nur vereinzelte Nachweise an Beifuß (Artemisia), Wegerich (Plantago), Grasnelke (Armeria), Steinbrech (Saxifraga), Ampfer (Rumex), Weidenröschen (Epilobium), Sonnenröschen (Helianthemum), Wiesenraute (Thalictrum) und verschiedenen Korbblütengewächsen (Liguliflorae, Tubuliflorae). Nicht autochthonen Ursprungs sind vermutlich ebenfalls häufiger erscheinende Heidekrautgewächse (Ericaceae p. p.), da ihre Kurve konform zu den umgelagerten Palynomorphen und hier insbesondere $\mathrm{zu}$ Fichte, Tanne und Hainbuche im weiteren Diagrammverlauf aussetzt. Auf eine mögliche Umlagerung holsteinzeitlicher Sedimente wurde bereits oben verwiesen - insbesondere in der Schattholzphase und am Ende der Warmzeit traten aufgrund der niederschlagsbedingten Bodenversauerung vermehrt Heidekrautgewächse in Erscheinung.

Dies trifft auch auf die dem unmittelbaren Standort zugerechnete Flora zu: Außer vermutlich aus älterwarmzeitlichen Ablagerungen stammenden Einzelnachweisen von Königsfarn (Osmunda), Großem Algenfarn bzw. bei Fehlen der artspezifischen Glochidien möglicherweise auch Gemeinem Schwimmfarn (Salvinia natans) und Farnen, wurden gelegentlich Ährentausendblatt (Myriophyllum spicatum), verschiedene Hahnenfußgewächse (Ranunculaceae) und Mondraute (Botrychium) beobachtet. Bei den Algen treten vor allem verschiedene Faden-Jochalgen, wie Schrauben- (Spirogyra) und Sternalgen (Zygnemataceae) sowie zu den Grünalgen zählende Zahnrädchenalgen ( $P e$ diastrum), insbesondere das kühlezeigende Pediastrum kawraiskyi in den Vordergrund. Daneben bestehen vereinzelte Nachweise an Schwammnadeln, Strudelwurmresten und Dauereiern von Rotatorien (hier die ebenfalls kühlezeigende Filinia hofmanni).

Für den Unterabschnitt der PZ A2 zeigt das Pollendiagramm (Abb. 10) zunächst einen kurzfristigen Rückgang präquartärer und älterwarmzeitlicher Sporomorphen. Die außer auf Umlagerung möglicherweise auch auf Fernflugeintrag basierenden Anteile der Kiefer bleiben davon nahezu unberührt. Konform erfolgt eine leichte Zunahme des NBP-Anteils, neben den bereits oben angeführten Einzelfunden im Wesentlichen verursacht durch Süß- und Sauergräser (wohl hauptsächlich aus dem unmittelbaren Standortumfeld stam- mend) sowie zungenblütige Korbblüten- (Liguliflorae) und Nelkengewächse (Caryophyllaceae). Eine erneute Häufung umgelagerten Materials, unter den älterwarmzeitlichen insbesondere der Fichte, Tanne, Hainbuche und der Erle, liegt für die Basis der Profilschiene 1a vor.

Innerhalb der standorteigenen Flora fallen, analog zum Wiederanstieg der Umlagerungen, besonders hohe Anteile an Farnen auf. Die im Übergang zum Saale-Spätglazial stattfindende Erosion unverfestigten Bodensubstrats, die möglicherweise das Fehlen der nachfolgenden saalespätglazialen PZ B mit verursachte, wird durch die leicht erhöhten Werte des Lebermooses Anthoceros punctatus unterstrichen. Zudem war der Sedimentationsraum anscheinend auch Wasserspiegelschwankungen unterworfen, da sich die Anteile explizit verschiedener Spirogyra-Arten und auch von Pediastrum kawraiskyi erhöhen und hier Ausdruck des weiter wirksam bleibenden und die Beckengenese beeinflussenden Randsenkendiapirismus sein könnten. Die temporäre Verflachung des Sedimentationsraumes reflektiert dann letztendlich vor allem die für das Saale-Spätglazial ermittelte Sumpfund Wasserflora (vgl. 4.3.3.2).

\subsubsection{Saale-Spätglazial}

PZ C1, Zeit der Birken-(Wacholder)-Sanddorn-Gemeinschaften Folgt man der bei STRAHL \& HERMSDORF (2008) vorgestellten Gliederung saalepleni- und saalespätglazialer Ablagerungen in den neuen Bundesländern, so fehlt dem Hauptprofil A die auf die waldlose Zeit des Saalepleniglazials folgende Phase der Sanddorngebüsche (PZ B). Aufgrund dieser Schichtlücke schließt sich der PZ A2 unmittelbar der Zeitraum einer ersten lichten Bewaldung des Untersuchungsgebietes an, die nach den Pollentypen hauptsächlich durch Baum- aber auch Zwergbirken (Betula nana) und eventuell Kiefern gebildet wurde (Abb. 10). Nachweise des Wacholders liegen aufgrund der oben angeführten schlechten Erhaltung des Sporomorpheninventars so gut wie nicht vor. Dass es sich um einen offenen, inselartigen Waldtyp handelt, der sich auch in dem von KREMENETSKI (2010: 275) publizierten Diagramm mit der LPZ 3 (dort als bereits eemzeitlich ausgewiesen!) widerspiegelt, zeigen sowohl die Beteiligung des Sanddorns, einem schattenintoleranten Rohbödenpionier, als auch die immens angestiegenen NBP-Anteile. Diese gehen überwiegend auf Süß- und Sauergräser, Beifuß sowie zungen- und röhrenblütige (Tubuliflorae) Korbblütengewächse zurück. Zu den weiteren, wesentlich seltener beobachteten Offenlandelementen zählen Knöterich (Polygonum), Enziangewächse (Gentianaceae), Weidenröschen, Nelken- und Gänsefußgewächse (Chenopodiaceae), Meerträubel (Ephedra), Kreuzblütengewächse (Cruciferae), Wegerich, Lein (Linum), Ampfer und verschiedene Rosengewächse (Rosaceae p. p.).

In den Uferbereichen bestand eine erste ausgeprägte Versumpfungstendenz. Diese äußert sich neben der Ausbreitung von zunächst Farnen [Gemeiner Sumpffarn (Thelypteris palustris)?, Artbestimmung aufgrund des durchweg fehlenden Perispors nicht möglich] in der anschließenden Ausbildung von Röhrichten mit Rohr- (Typha) und/oder Igelkolben (Sparganium). Die Entwicklung eines Schwimmblattgürtels mit Seerosengewächsen (Nymphaeaceae) sowie von Wasserschwebergesellschaften mit Gemeinem Schwimmfarn und Wasserlinse (Lemna sp., vgl. LPZ 3 KREMENETSKI 2010), 
beides Gattungen mit in der Regel hohem Nährstoff- und Sommerwärmeanspruch, signalisieren eine zumindest kurzfristige Besiedlung des Verlandungsbereichs durch Höhere Wasserpflanzen. Diese bricht jedoch in NN2 mit dem (nicht graduellen) Übergang in die eemzeitlichen Ablagerungen ab.

Der Anteil umgelagerter präquartärer Sporomorphen erreicht letztmalig mehr als $20 \%$ und geht dann auf zu vernachlässigende Werte zurück. Zunächst davon nicht betroffen sind thermophile Gehölze wie Ulme, Hasel, Erle und Hainbuche im Gegensatz zu Kiefer, Fichte und Tanne. Bezüglich der heterogenen Zusammensetzung der Pollen- und Sporenflora und des Nebeneinanderexistierens geschichteter und verwürgter Schluffpartien vor allem im Übergang der Profilschienen 1a und 1 bestünde zum einen die Möglichkeit der Durchmischung saalespätglazialer mit eemzeitlichen Ablagerungen. Zum anderen kommt aber auch die Aufarbeitung weiterer, nun allerdings älterer, an Kiefer und anderen Nadelgehölzen, explizit an Tanne ärmerer holsteinzeitlicher Ablagerungen (älter PZ 5-7 nach ERD 1973) in Betracht. Dafür sprächen sowohl die vergleichsweise hohen Hainbuchenanteile, die dem frühen Eem definitiv fehlen und der deutlich höhere Inkohlungsgrad der Sporomorphen gegenüber den nachfolgenden eemzeitlichen Ablagerungen. Demnach existiert ein weiterer, zwischen dem Saale-Spätglazial und dem Eem liegender Hiatus. Dieser beinhaltet sowohl den kiefernreichen Abschnitt des ausgehenden Saale-Spätglazials (PZ C2 nach STRAHL \& HeRMSDORF 2008) als auch die Warmzeit einleitende, ausgeprägte Birken-Phase (PZ 1 nach ERD 1973), der noch jegliche thermophile Gehölze und in der Regel auch weitestgehend größere Kiefernbestände fehlen.

Für den Zeitraum des Saale-Pleni- und des Saale-Spätglazials bestehen gute Vergleichsmöglichkeiten mit dem von Kremenetski (2010) bearbeiteten Pollenprofil. So reflektieren die LPZ 1-2 wie im Hauptprofil A das ausgehende Saale-Glazial (PZ A), auch hier stark mit resedimentierten präquartären und älterwarmzeitlichen Sporomorphen belastet und die LPZ 3 entsprechend der PZ C1 eine erste Birkenausbreitung nebst einer ebenfalls deutlich abzulesenden Verlandungstendenz. Das Profil umfasst allerdings insgesamt, inklusive der eemzeitlichen Ablagerungen, eine entsprechend seiner beckenrandlichen Position nur 5 m mächtige Sedimentabfolge. Die eemzeitliche Vegetationsentwicklung wird tatsächlich erst ab der LPZ 4 (= PZ 4, oberer Teil nach ERD 1973) mit der Etablierung von Haselbeständen und damit einem zeitlich gesehen noch umfangreicheren Hiatus als im Hauptprofil A abgebildet.

\subsubsection{Eem-Interglazial}

\section{PZ 2, Kiefern-Birken-Zeit}

Der hier erste, eemzeitlich einzustufende, aber nur durch eine Probe belegte Vegetationsabschnitt ist in der Regel seitens der Gehölze durch die Kiefer und die Ulme sowie im Übergang zur PZ 3, die beginnende Einwanderung der Eiche geprägt. Die Birke weist einen letztmaligen prozentualen Anteil von knapp $30 \%$ auf. Im Pollendiagramm (Abb. 10) setzen die Kurven von Hasel, Erle, Fichte und Tanne völlig aus, Hainbuche und präquartäre Sporomorphen sind weiter rückläufig. Auffällig ist der weiterhin hohe Anteil an Offenlandelementen (34\%), hauptsächlich bestehend aus Süß- und Sauergräsern, gegenüber der PZ C1 nur untergeordnet Bei- fuß, Korbblütengewächsen, Wegerich, Nelken- und Gänsefußgewächsen.

Inwiefern im Vergleich mit dem Becken NN1 auch gewisse Salzeinflüsse eine Rolle spielten, konnte nicht geklärt werden, da sowohl u. a. dahingehend relevante Makroreste als auch Diatomeen fehlten. Ebenso fielen die für einen diesbezüglichen Nachweis durchgeführten Analysen zur Leitfähigkeit (Lf) sowie zu den Gehalten an $\mathrm{SO}_{4}^{2-}$ und $\mathrm{Cl}^{-}$(vgl. Tab. 3) im Ergebnis zu niedrig aus. Eine leichte Zunahme von $\mathrm{SO}_{4}^{2-}$ gegenüber den Profilschienen 1 und 2 ist lediglich in Profilschiene 5 feststellbar, liegt dort aber im Bereich von sekundären Gipsausblühungen. Hinweise liefern bisher lediglich das Auftreten von c.f. Hydrobia sp. (vgl. 4.3.4) innerhalb der PZ 5 sowie die von der Universität Leiden (mündl. Vortragsmitt. C. BAKELs) bei ihren parallelen Untersuchungen in NN2 ermittelten spärlichen Makroreste. Diese erbrachten zumindest Exemplare der als obligater Halophyt geführten Meeressalde (Ruppia maritima) und des als fakultativen Halophyten geltenden Sumpfteichfadens (Zannichellia palustris).

Ebenso wie für das Saale-Spätglazial und die beginnende Eem-Warmzeit ist bezüglich der Diagrammlage (Abb. 10) ein gradueller Übergang in die PZ 3 nicht abzuleiten. Vielmehr besteht am hiesigen Profilentnahmepunkt ein weiterer Hiatus, der den Zeitraum der eemzeitlichen Kiefern-Eichenmischwald-Zeit nahezu vollständig umfasst. Die Ursachen liegen in den Sedimentationsumständen, verbunden mit sehr geringen Sedimentationsraten bzw. in der Wiederaufarbeitung entsprechender Sedimente.

\section{PZ 3/4, Kiefern-Eichenmischwald-Zeit/Eichenmischwald- Hasel-Zeit}

Der den Übergangsbereich der PZ 3 und 4 bildende Abschnitt im Pollendiagramm (Abb. 10) wird durch Kiefer, Ulme und Eiche geprägt. Gegen eine ausschließliche Einstufung in die PZ 3 sprechen die bereits maximal $14 \%$ erreichenden HaselWerte. Erhaltungsbedingt wurde die für diesen Zeitraum weitaus häufiger zu erwartende Esche nur vereinzelt beobachtet. Ebenfalls mehr für eine Zuordnung zur PZ 4 sprechend, setzen die Kurven von Erle und Fichte erneut ein, wohingegen endgültig Hainbuche und Tanne konform mit den noch maximal 3-5 \% erreichenden präquartären Sporomorphen vorläufig ausklingen. Weiterhin hoch bleiben die NBPAnteile in der zuvor genannten Zusammensetzung. Hinzu kommen neben nun deutlich mehr Beifuß und Gänsefußgewächsen Vogelknöterich (Polygonum aviculare) als Vertreter frischer Ruderal- und Trittgesellschaften, vereinzelt Krähenbeere (Empetrum), Heidekraut (Calluna), Glockenblume (Campanula), Kreuzblütengewächse, Steinbrech, verschiedene Rosen- und Schmetterlingsblütengewächse (Fabaceae), Mädesüß (Filipendula), Wiesenraute, Labkraut (Galium), Doldenblüten- (Umbelliferae) und Enziangewächse. Dies spricht - wie im Becken NN1 (MAI in MANIA 2000: 74-75) - im Gegensatz zur normalerweise dichten eemzeitlichen Bewaldung, für einen steppenwaldartigen Charakter der Vegetation in der weiteren Umgebung des Beckens NN2, der zumindest in dieser ausgeprägten Form bis zur Massenausbreitung der Hasel in der PZ 4 erhalten blieb. Nach letztmaligem Nachweis von Resten des Gemeinen Schwimmfarns innerhalb der standorteigenen Flora in der PZ 2 kommt es nun auch zum Ausklingen von Rohr- bzw. Igelkolben, lediglich 
Tab. 3: Analysenergebnisse zur Untersuchung der Leitfähigkeit (Lf) sowie des Gehaltes an $\mathrm{SO}_{4}^{2-}$ und Cl an Stichproben aus den Profilschienen 1a-6 des Hauptprofils A, Becken NN2 (Analyse: Landeslabor Berlin-Brandenburg, FB U2/Geochemie, Kleinmachnow).

Tab. 3: Results of analysis of conductivity (Lf) and of content of $\mathrm{SO}_{4}^{2-}$ and $\mathrm{Cl}^{-}$in random samples from partial profiles 1 a-6 of main profile A, basin $\mathrm{NN2}$ (analysis: regional laboratory Berlin-Brandenburg, FB U2/Geochemie, Kleinmachnow).

\begin{tabular}{|l|l|l|l|l|l|l|}
\hline Ident-Nr. & Pr. Nr. & Labor-Nr. & $\begin{array}{l}\text { Lf [1:5] } \\
\boldsymbol{\mu S / m}\end{array}$ & $\begin{array}{l}\mathbf{L f}[\mathbf{1 : 1 0}] \\
\mathbf{S S} / \mathbf{m}\end{array}$ & $\begin{array}{l}\mathbf{S O}_{4}^{2-} \\
\mathbf{m g} / \mathbf{k g}\end{array}$ & $\begin{array}{l}\mathbf{C l}^{-} \\
\mathbf{m g} / \mathbf{k g}\end{array}$ \\
\hline 100090324 & $1 \mathrm{a} / 1$ & 6444 & - & 160 & 19 & 7 \\
\hline 100090325 & $1 / 3$ & 6445 & 1677 & - & 970 & 5 \\
\hline 100090326 & $2 / 5$ & 6446 & 1514 & - & 814 & 6 \\
\hline 100090327 & $3 / 7$ & 6447 & 384 & - & 111 & 6 \\
\hline 100090328 & $4 / 9$ & 6448 & 650 & - & 257 & 5 \\
\hline 100090329 & $5 / 10$ & 6449 & 1962 & - & 1200 & 15 \\
\hline 100090330 & $6 / 13$ & 6450 & 299 & - & 149 & 12 \\
\hline
\end{tabular}

die Kurve der monoleten Farnsporen ist mit deutlich niedrigeren Werten weiterhin zu verfolgen, was hinsichtlich der Beckenkonfiguration für einen insgesamt nur spärlichen Bewuchs der möglicherweise zu schmalen und/oder zu steilen Uferbereiche spricht.

\section{PZ 4, Eichenmischwald-Hasel-Zeit}

Die PZ 4 umfasst den Hauptteil des archäologischen Fundhorizontes NN2/2 (Abb. 4). Die Abgrenzung dieses Vegetationsabschnitts zum vorhergehenden Übergangshorizont erfolgt anhand des Eem-typischen Steilanstiegs der Hasel-Kurve, der aufgrund der zu Beginn immer noch sehr hohen NBPAnteile (jetzt vor allem Süßgräser, Vogelknöterich, zungenblütige Korbblüten- und Nelkengewächse) abgeschwächt erscheint. Birke und Kiefer spielen für den Waldaufbau keine Rolle mehr, im Gegensatz zu den Eichenmischwaldkomponenten, unter denen Eiche und Ulme zu Beginn der Massenausbreitung der Hasel Maximalwerte um $20 \%$ erreichen. In diesen Zeitraum fällt auch der endgültige Kurvenschluss der Erle, deren Pollenfrequenzen aber während des gesamten Interglazials die $5 \%$-Marke nicht überschreiten. Damit ist, abgesehen von der Bestockung höchstens kleinerer Feuchtareale, von einem Fehlen ausgedehnter Bruchwaldbestände in der unmittelbaren Umgebung des Paläosees auszugehen. Ferner ist die Linde zwar mehr oder weniger durchgehend nachweisbar, ohne jedoch Werte über $1 \%$ zu erreichen. Wie die Esche ist auch die Eibe erhaltungsbedingt nur ganz vereinzelt zu beobachten, was insbesondere die Abgrenzung zur anschließenden PZ 5 deutlich erschwert. Die Fichtenwerte verbleiben stets unter $2 \%$ und gehen somit zu diesem Zeitpunkt ausschließlich auf Fernflugeintrag zurück (vgl. LANG 1994). Unter den Elementen mit einer ozeanischen Verbreitungstendenz sind vor allem Efeu (Hedera) und die auf den unmittelbaren Standortbereich beschränkte Binsenschneide (Cladium mariscus) hervorzuheben. Sporadisch erscheint der Ahorn (Acer), wobei wegen der fehlenden Makroreste kein Rückschluss auf eine eventuelle Etablierung des Tatarischen Ahorns (Acer tataricum) entsprechend NN1 möglich ist (vgl. 5.2).

Einen wiederholten Hinweis auf zeitweilige Schwankungen des Seespiegels liefern die kurzzeitig ansteigenden Werte von Pediastrum boryanum sowie verschiedener SpirogyraArten. Sie fallen mit der Ausbildung von Fließgefügen innerhalb des oberen Teils der Profilschiene 1 zusammen und deuten auf eine rasche Sedimentakkumulation unter Flachwas- serbedingungen hin. Auf bis in die Profilschiene 1 hinein zu verfolgende, sukzessive nachlassende Schichtdeformationen folgt das Einsetzen monotoner, offensichtlich rasch zusammengeschwemmter Ablagerungen, der im Pollendiagramm die gestreckt wirkenden PZ 4 und vor allem 5 entsprechen. Darin ist stets ein gewisser Anteil umgelagerter präquartärer Sporomorphen festzustellen, der erst im Bereich der Profilschienen 4 und 5 (Abb. 10) beinahe völlig ausbleibt.

Insbesondere zu dem von KREMENETSKI (2010) untersuchten Profil lassen sich bezüglich des oberen Teils der PZ 4 (= LPZ 4 Kremenetski) Parallelen ziehen. Wie im Hauptprofil A liegt eine geringe Beteiligung von Linde und Erle sowie ein nahezu gänzliches Fehlen der Eibe bei synchron erhöhten NBP-Anteilen vor, so dass auch hier eine eindeutige Zonierung des Pollendiagramms erschwert wird.

PZ 4/5, Eichenmischwald-Hasel-Zeit/Hasel-Eiben-Linden-Zeit Aufgrund der in NN2 und auch NN1 generell schwachen, wahrscheinlich standörtlich bedingten Lindenbeteiligung und des in NN2 erhaltungsbedingten, nahezu völligen Fehlens der Eibe, kann die Abgrenzung der PZ 5 sowohl im Hauptprofil A als auch in dem von KREMENETSKI (2010) publizierten nicht anhand des charakteristischen Ansteigens zunächst der Eiben- und nachfolgend der Linden-Kurve vorgenommen werden. Sie muss sich hier an anderen Merkmalen orientieren. Für den im Hauptprofil A (Abb. 10) ausgewiesenen Übergangsbereich zwischen beiden PZ liegt ein erster deutlicher Abfall der Werte von Ulme und Eiche, die Hasel zeigt sich noch unbeeinflusst. Während die Linde nach wie vor Werte unter $1 \%$ aufweist konnte die Eibe lediglich einmal beobachtet werden. Innerhalb der Offenlandflora gehen insbesondere Knöterich und Nelkengewächse zurück, Süßund Sauergräser bleiben mehr oder weniger konstant vertreten. Eine Wiederausbreitungstendenz besteht bei den Gänsefußgewächsen und an der Obergrenze des Abschnittes auch beim Beifuß und röhrenblütigen Korbblütengewächsen. Bemerkenswert ist eine leichte Häufung der Glockenblume.

\section{PZ 5, Hasel-Eiben-Linden-Zeit}

Die Abgrenzung der PZ 5 als solche erfolgt letztendlich anhand des ersten deutlichen Abfalls der Hasel-Kurve, der mit einem Wiederanstieg der Kurven von Eiche und Ulme sowie nur kurzzeitig von Kiefer und Hainbuche koinzidiert (Abb. 10). Die in diesem Waldstadium einwandernde Hainbuche erreicht jedoch erst im Übergang zur PZ 6 Kurven- 
schluss. Die Fichte weist nahezu durchgehend Werte über 1-2\% auf und steigt endgültig, zusammen mit der Kiefer, im oberen Teil der PZ 5 kontinuierlich an. Das für diesen Zeitraum zu erwartende Linden-Maximum verschiebt sich hier in die PZ 6, liegt jedoch typisch für das Eem zwischen der Hasel- und Hainbuchenmaximalverbreitung. Einen nahezu entsprechenden, jedoch nur den oberen Teil der PZ 5 reflektierenden Verlauf zeigt auch das Diagramm des wegen seiner randlichen Position weniger hochaufgelösten Profils von KREMENETSKi (2010).

Ein erster, deutlicher Anstieg der Kiefern-Kurve wie auch eine leichte, nicht anhaltende Ausbreitungstendenz bei Birke und Hainbuche fällt zu Beginn der PZ 5 zunächst mit einer erneuten Zunahme des NBP-Anteils zusammen. Diese geht vor allem auf Süß- und Sauergräser, Beifuß, Gänsefuß- und röhrenblütige Korbblütengewächse sowie untergeordnet Knöterich und Nelkengewächse zurück. Ein Fernflugeintrag der genannten Gehölze in die recht offene Waldlandschaft ist damit nicht auszuschließen, so nicht ein rein statistischer Effekt aufgrund des zeitgleichen kurzfristigen Haselrückgangs zugrunde liegt (schriftl. Mitt. K.-E. BEHRE). Als Waldbegleiter tritt unter den Farnen der Gemeine Adlerfarn (Pteridium aquilinum) in den Vordergrund. Ebenso häufen sich in diesem Diagrammabschnitt die Funde von Pollen der Ackerwinde (Convolvulus), die SeIFERT-Eulen (2010) neben den zunehmenden NBP für den anhaltenden Einfluss von Steppenelementen in NN1 anführt. Durch die nachfolgende allmähliche Unterwanderung der Hasel-dominierten Laubmischwaldgesellschaften durch die Fichte und anschließend die Hainbuche im höheren Teil der PZ 5 gehen die NBP-Anteile deutlich zurück und basieren im Wesentlichen nur noch auf Süßgräsern und Gänsefußgewächsen.

Die offensichtliche Etablierung von Riedgemeinschaften (Sauergräser) sowie das wiederholte Erscheinen glatter Zygosporen von verschiedenen Spirogyra-Arten im unteren Teil der PZ 5 lässt auf die allmähliche Einstellung von Flachwasserbedingungen schließen. Dass dabei auch wieder erosive Prozesse eine Rolle spielten, zeigt das bis hierhin kontinuierliche Auftreten präquartärer Sporomorphen bis in den Bereich der Profilschiene 3 (Abb. 10) sowie der insgesamt sehr schlechte Erhaltungszustand der Palynomorphen. Im oberen Teil des Abschnitts treten anstelle von Seggenrieden Röhrichte mit Rohr- oder Igelkolben sowie, zunächst noch untergeordnet, Breitblättrigem Rohrkolben (Typha latifolia).

\section{PZ 6, Hainbuchen-Zeit}

Die Untergrenze der PZ 6 wird mittels des Anstiegs der Hainbuchen-Kurve auf über $20 \%$ festgelegt. Ungewöhnlich für eemzeitliche Ablagerungen ist eine der Massenausbreitung der Hainbuche vorangehende erste Fichtenausbreitung, die auch Seifert (1990, Abb. 11b) und nachfolgend KREMENETSKi (in Boettger et al. 2005, 2007, Abb. 11d) für das Becken NN1 feststellten (vgl. 5.2). Das nahezu gleiche Bild vermittelt in NN2 das Diagramm von KREMENETSKI (2010), wobei das Fichten-Maximum hier gegenüber dem Hauptprofil A prozentual deutlich höher ist und mit einem Peak der Kiefern-Kurve zusammenfällt. Angesichts der bei tatsächlich korrekter Bestimmung ebenfalls kulminierenden Kurven der Wasserlinse (Lemna) sowie analog zum Hauptprofil A (Abb. 10) von Rohr- und Igelkolben wäre diese zusätzliche Häufung einerseits als mögliche Folge der Anreicherung in- nerhalb von Spülsäumen in extrem flachen Wasserbereichen deutbar. Andererseits ist auch die Förderung der Fichte gegenüber der Hainbuche aufgrund der Vernässung ufernaher Areale der engeren Standortumgebung ins Kalkül zu ziehen. Die eigentliche Ursache der in beiden Becken festgestellten frühen Fichtenausbreitung ist jedoch vermutlich in der räumlichen Nähe von Fichten-besetzten Mittelgebirgs-standorten zu suchen. Von diesen wäre eine rasche Expansion hin zu Standorten denkbar, die zu dieser Zeit einer Entwicklung von an Edellaubhölzern reichen Laubmischwäldern hin zu durch Hainbuchen dominierten Schattholzwäldern unterlagen. Einen vergleichbaren Diagrammverlauf zeigen, wenn auch auf niedrigerem Niveau, Profile aus der Lausitz (Lug, ERD 1979; Reddern, ERD \& STRAHL 2008; Tschernitz, STRAHL 2004) und aus dem Fläming (Nedlitz, STRAHL 2007; alle in STRAHL \& HERMSDORF 2008), ohne dass sich die Fichte jedoch an einem dieser Standorte dauerhaft hätte gegen die Hainbuche durchsetzen können. Vielmehr ist diese scheinbare Expansion in der Mehrzahl der vorliegenden Untersuchungen an einen Peak der NBP-Kurve (vorwiegend Süßgräser sowie Beifuß und Gänsefußgewächse) gebunden und somit wohl eher ein Fernflugphänomen (vgl. LiтT 1994a).

Unter den übrigen Laubmischwaldbildnern erlebte die Ulme eine Renaissance, während die Werte der Linde erstmals die $1 \%$-Marke überschreiten. Vor allem die Hasel und hier im Unterschied zu NN1 (vgl. 5.2) vermutlich erhaltungsbedingt die Eiche waren dagegen deutlich rückläufig. Mit der Massenausbreitung der Hainbuche verlor die Bewaldung definitiv ihren aufgelockerten bzw. inselartigen Charakter. Nach einer letztmaligen Kulmination der Süßgräser wurden Offenlandelemente in der Umgebung von NN2 bis zum Beginn des Weichsel-Frühglazials mehr oder weniger bedeutungslos.

\section{PZ 7, Hainbuchen-Fichten-Zeit}

Die PZ 7 hebt sich als ein absolut durch die Hainbuche dominiertes Stadium der eemzeitlichen Waldentwicklung heraus. Wichtigstes Nadelgehölz ist bezüglich der zwei bisher aus dem Becken NN2 veröffentlichten Pollendiagramme die Fichte, ohne aber an ihre zu Beginn der PZ 6 erreichten Werte nochmals anschließen zu können. Während sämtliche Edellaubhölzer und Offenlandelemente anscheinend durch die Verschattung der Waldstandorte bedeutungslos wurden, konnte sich die Ulme auf stauwassergesättigten Böden noch weiterhin halten. Dabei dürfte die bessere Erhaltungsfähigkeit ihres Pollens gegenüber dem der Eiche und damit seine sekundäre Anreicherung auch eine Rolle gespielt haben.

Mit der Umstellung der Sedimentation von Schluff- zu Detritusmudde schreitet die Verflachung des Seebeckens in diesem Zeitraum weiter voran. In den entstehenden, auch seitens der Mollusken belegten (vgl. 4.3.4) Flachwasserbereichen breitete sich zunehmend Pediastrum boryanum aus, das in den PZ 8-9 seinen Verbreitungsschwerpunkt erreichte. Die noch bis in die PZ 7 hinein die Uferzonen des Beckens beherrschenden Röhrichtgemeinschaften wurden dagegen durch Farne verdrängt.

\section{PZ 8, Kiefern-Fichten-Tannen-Zeit}

Infolge der extremen Verflachung des Sedimentationsraums, auf die indirekt anhand der Massenausbreitung von Pediastrum boryanum geschlossen wird, sind für diesen Abschnitt 
offensichtlich nur noch extrem geringe Sedimentationsraten beim Absatz von nun Kalk- und Algenmudde zu verzeichnen. So umfasst die durch die zunehmende Ausbreitung der Nadelgehölze, insbesondere der Tanne (hier ermittelter Maximal-Wert $18 \%$ ) gekennzeichnete PZ 8 im Hauptprofil A (Abb. 10) gerade einmal $2 \mathrm{~cm}$. Ebenso ist dieser Abschnitt im Pollendiagramm von KRemenetski (2010) durch nur eine Probe belegt.

Die Hainbuche erreicht im Hauptprofil A letztmalig einen Wert von $70 \%$ und geht danach scheinbar schlagartig zurück. Ein üblicherweise sukzessives Ausklingen kann aufgrund des extrem schlechten Erhaltungszustandes der Sporomorphen in den oberhalb der beiden auswertbaren entnommenen Proben nicht nachverfolgt werden. Die Pollenspektren sind im Wesentlichen durch das massenhafte Vorkommen von Pediastrum boryanum und monoleten Farnsporen, die ab der PZ 8 die Sumpfvegetation dominieren, geprägt.

\section{PZ 9, Kiefern-Zeit}

Aus den oben genannten Gründen konnten lediglich drei Proben für das abschließende eemzeitliche Waldstadium zu einer Auswertung herangezogen werden. Dem Profil ist ebenso wie dem von Kremenetski (2010) untersuchten die charakteristische Wiederausbreitung der Kiefer gemein, die klimatisch ausgelöst, zu einer Verdrängung der übrigen, insbesondere wärmeanspruchsvolleren Gehölze führte.

Insgesamt legt die Umstellung auf die Sedimentation von Kalkmudde ein Wiedervernässen des Sedimentationsraums infolge eines generellen Wasserspiegelanstiegs nahe, der zur Einschränkung versumpfter, weiterhin durch Farne beherrschter Standorte führte. Jedoch blieb es insgesamt bei einer Beibehaltung von Flachwasserbedingungen. Dafür sprechen sowohl der hohe Anteil der Wasserlinse (KREMENETSKI 2010) als auch die weiterhin weit über $100 \%$ erreichenden Anteile von Pediastrum boryanum (Abb. 10).

\subsubsection{Weichsel-Frühglazial}

Die letzte, aus dem Hauptprofil A statistisch auswertbare Probe lieferte ein Pollenspektrum, das nach seiner $\mathrm{Zu}^{-}$ sammensetzung bereits in das Weichsel-Frühglazial datiert (Abb. 10). Es ist durch den Rückgang der Kiefer charakterisiert, der durch eine Wiederausbreitung von Offenlandelementen, hier vor allem Süßgräser und zungenblütige Korbblütengewächse, begleitet wird. Des Weiteren erscheinen erneut thermophile Gehölze, vor allem Hainbuche, aber auch Fichte, die auf Resedimentation aus den jüngeren eemzeitlichen Ablagerungen zurückgehen. Eine weitere Verfolgung der weichselfrühglazialen Entwicklung ist aufgrund der an Palynomorphen sterilen Proben aus dem Hangendaufschluss vom Dezember 2007 (Proben W1 bis W4, vgl. Abb. 4) nicht möglich.

\subsubsection{Malakologie [S. Meng]}

Insgesamt sind im Becken NN2 ca. 50 Molluskenarten mit über 21000 Individuen festgestellt worden. Im Hauptprofil A konnten etwa 45 Arten und rund 10000 Individuen erfasst werden. Neben diesem Material werden auch Probennahmen aus den Jahren 2003 bis 2005, welche sich hauptsächlich auf den Bereich des archäologischen Hauptfund- horizontes NN2/2 am nördlichen Beckenrand konzentrieren, vergleichend berücksichtigt.

Dominiert werden die Faunen entsprechend dem Ablagerungsraum vor allem von limnischen Mollusken. Dagegen sind terrestrische Komponenten weniger vertreten (Abb. 12, Taf. 1, Tab. 4).

Aus der Rammkernsondierung stand nur die bereits visuell Molluskenschalen führende Probe U 5/6 für malakologische Untersuchungen zur Verfügung. Die pollenanalytisch in das Saale-Spätglazial datierten Schluffe führen mit Bithynia tentaculata, Gyraulus laevis sowie Fragmenten von Sphaerium corneum und Lymnaeidae eine arten- und individuenarme Süßwasserfauna. Kaltzeitliche terrestrische Elemente konnten nicht belegt werden. Das Artenspektrum leitet gut zur eemzeitlichen Fauna im Hangenden über.

Entsprechend der Probe U 5/6 aus dem Liegenden sind auch in den Proben 1 und 2 von der Basis des Schurfprofils nur wenige Individuen von Süßwassermollusken, beispielsweise mit Bithynia tentaculata, Valvata piscinalis und Gyraulus laevis enthalten.

Der Übergang vom Saale-Spätglazial zum Eem wird nach den Ergebnissen der Pollenanalyse von den Proben 3 und 4 dokumentiert. Charakterisiert wird die Süßwasserfauna wieder von Bithynia tentaculata, Gyraulus laevis und Valvata piscinalis, was insgesamt für eine relativ einheitliche Entwicklung des Gewässers spricht (siehe unten). Weitere Elemente sind Gyraulus crista, Hippeutis complanatus, Bathyomphalus contortus und Radix cf. balthica. Es handelt sich um eine reine Stillwasserfauna.

Auffällig und für das untersuchte Profil bezeichnend sind die geringen Abundanzen der Kleinmuscheln Pisidium und Sphaerium. Möglicherweise spricht dies für eine erhöhte Sauerstoffuntersättigung im Bodensubstrat des Gewässers, gegen welche Kleinmuscheln besonders empfindlich reagieren. Ein weiteres Indiz hierfür ist auch die Arten- und Individuenarmut von Süßwasserarten insgesamt, die über weite Teile des Profils anhält. Neben Sphaerium corneum konnten Pisidium subtruncatum und Pisidium nitidum nachgewiesen werden.

Die Proben 3 und 4 enthalten mit etwa 21 Arten innerhalb des Profiles die umfangreichste terrestrische Fauna. Der Reichtum terrestrischer Elemente ist hier keineswegs klimatisch zu interpretieren, sondern steht viel eher in Abhängigkeit sedimentologischer Prozesse oder zumindest in Bezug zum Ablagerungsraum. Dominiert wird die Fauna ausgehend von den terrestrischen Vertretern mit $95 \%$ der Individuen und $50 \%$ der Arten durch Elemente des Offenlandes, z. B. mit Vallonia costata, Vallonia pulchella, Vallonia excentrica, Pupilla muscorum, Truncatellina cylindrica und Vertigo pygmaea. Mit Chondrula tridens und Pupilla triplicata sind zudem auch charakteristische Steppenelemente vertreten. Anspruchsvollere thermophile Waldarten fehlen. Allerdings verweisen einige Nachweise von Fruticicola fruticum, Euomphalia strigella, Cepaea sp. und Arianta arbustorum zumindest auf lichte Gehölzbestände. Ubiquisten, die ebenfalls in Gehölzen vorkommen, sind beispielsweise mit Nesovitrea hammonis und Vitrina pellucida vertreten. Feuchtigkeitsliebend sind weniger als $1 \%$ der Individuen und etwa $10 \%$ der Arten. Vereinzelt fanden sich Gehäuse von Vertigo antivertigo und Vertigo angustior. Vermutlich 


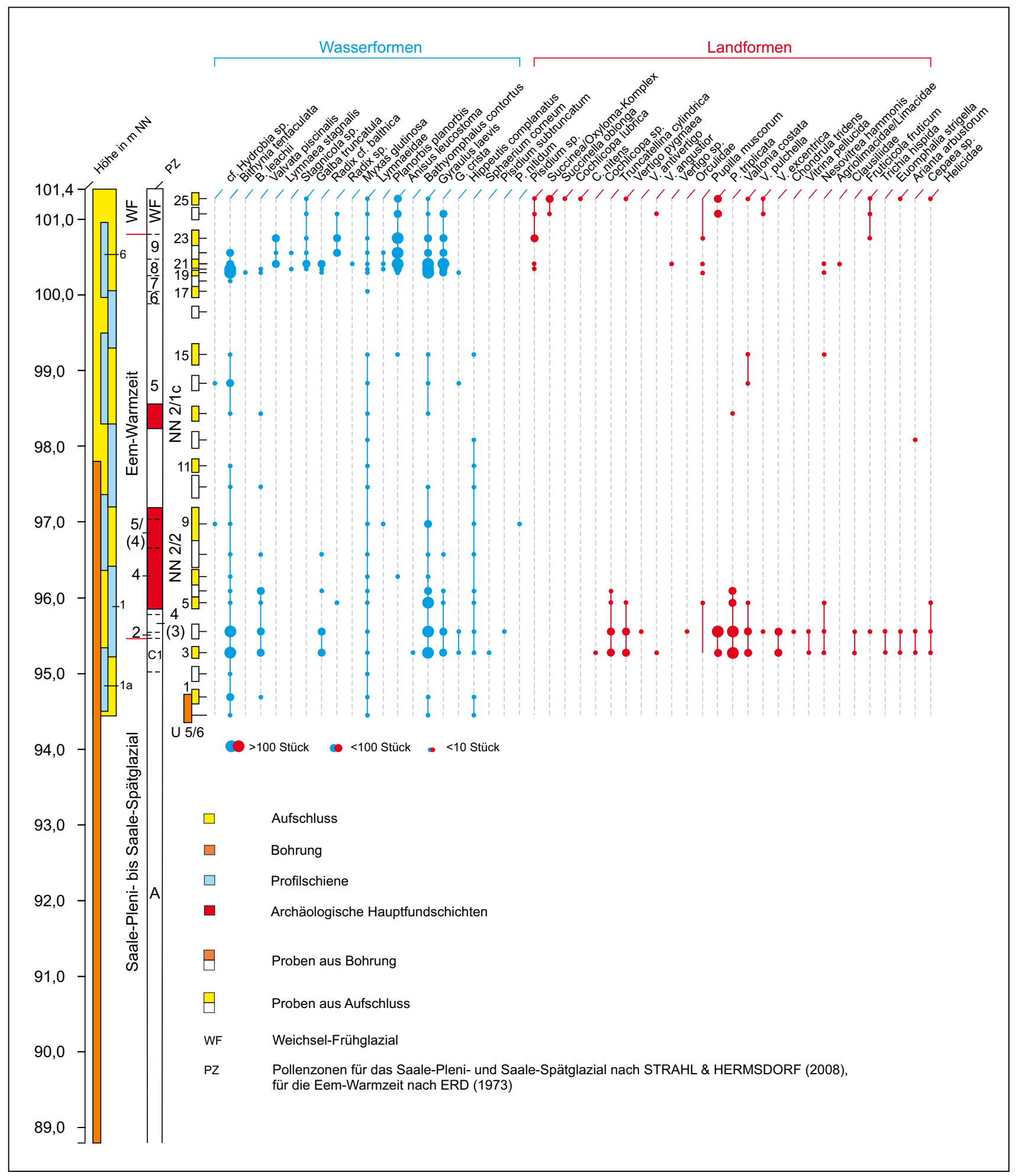

Abb. 12: Die Molluskenfauna aus dem Hauptprofil A, Becken NN2.

Fig. 12: Mollusc fauna of main profile A, basin NN2.

waren im Umfeld des Sees keine ausgedehnten Feuchthabitate entwickelt, was evtl. auf steilere Uferböschungen zurückgeführt werden kann.

In Probe 4 wurde das Gehäusefragment einer Landschnecke gefunden, welches bisher nicht näher determiniert werden konnte. Für das Quartär von Mitteleuropa handelt es sich dabei möglicherweise um einen neuen Exoten (Taf. 2). Die größte Übereinstimmung besitzt der Gehäuserest mit einer kleinen Orculidae, einer Gruppe, die heute vor allem im südöstlichen Europa verbreitet ist. Ausgeschlossen werden können allerdings die im Pleistozän Mitteleuropas verbreiteten Orculidae Sphyradium doliolum und Pagodulina pagodula. Das Gehäuse ist etwa $2 \mathrm{~mm}$ breit, zylindrisch und besitzt nur langsam anwachsende Umgänge. Die Pupillidae, im Wesentlichen Pupilla, sind in der Regel kleiner und haben deutlich niedrigere Windungshöhen. Keine Entsprechungen fanden sich auch bei den Enidae und Chondrinidae. Problematisch ist, dass bei dem Fragment keinerlei Ansätze von Zahnlamellen erkennbar sind. Folglich können diese bei der vorliegenden Art innerhalb der Mündung nur relativ kurz 


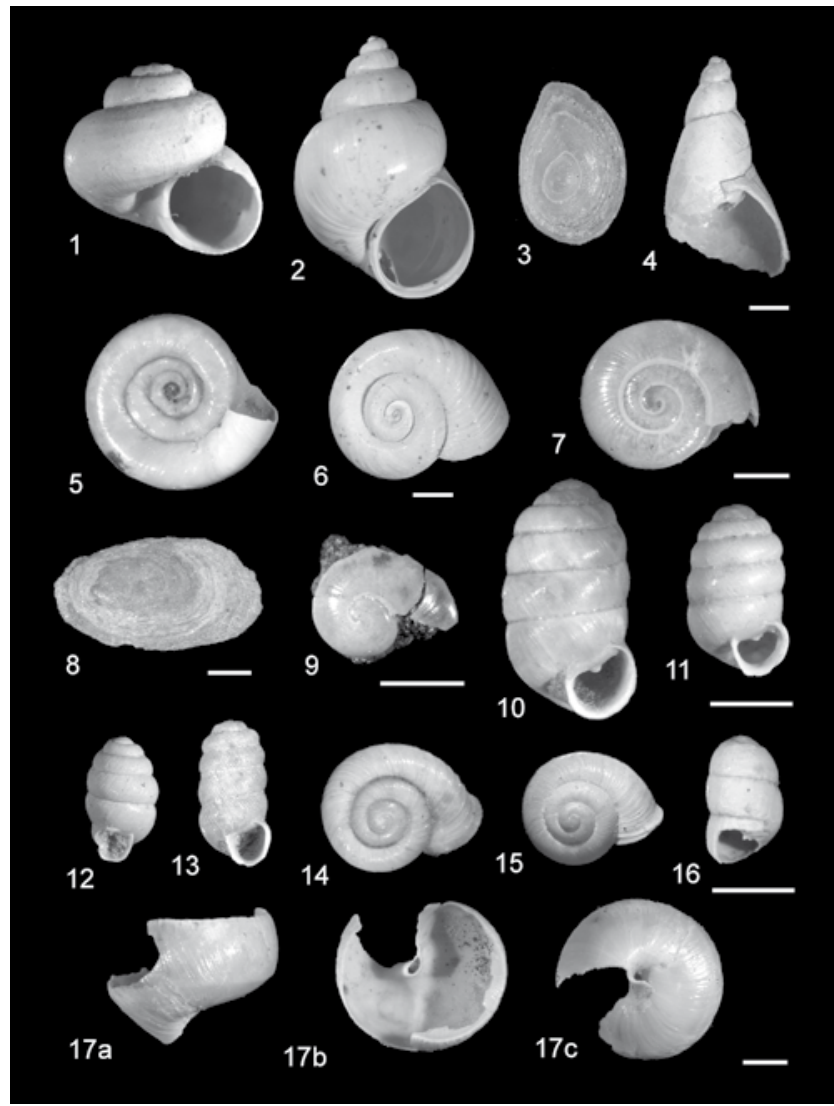

Taf. 1: Mollusken aus dem Hauptprofil A, Becken NN2.

1: Valvata piscinalis (Pr. 4), 2: Bithynia tentaculata (Pr. 4), 3: B. tentaculata Deckel (Pr. 4), 4: Lymnaea stagnalis (Pr. 21), 5: Anisus leucostoma (Pr. 21), 6: Gyraulus laevis (Pr. 4), 7: Nesovitra hammonis (Pr. 4), 8: Limacidae/Agri olimacidae (Pr. 4), 9: Vitrina pellucida (Pr. 4), 10: Pupilla muscorum (Pr. 3), 11: Pupilla triplicata (Pr. 4), 12: Vertigo angustior (Pr. 3), 13: Truncatellina cylindrica (Pr. 4), 14: Vallonia costata (Pr. 4), 15: Vallonia excentrica (Pr. 4), 16: Clausiliidae (Pr. 21), 17: Chondrula tridens (Pr. 4).

Plate 1: Molluscs of the main profile A, basin NN2.

1: Valvata piscinalis (sample 4), 2: Bithynia tentaculata (sample 4), 3: B. tentaculata, Deckel (sample 4), 4: Lymnaea stagnalis (sample 21), 5: Anisus leucostoma (sample 21), 6: Gyraulus laevis (sample 4), 7: Nesovitra hammonis (sample 4), 8: Limacidae/Agriolimacidae (sample 4), 9: Vitrina pellucida (sample 4), 10: Pupilla muscorum (sample 3), 11: Pupilla triplicata (sample 4), 12: Vertigo angustior (sample 3), 13: Truncatellina cylindrica (sample 4), 14: Vallonia costata (sample 4), 15: Vallonia excentrica (sample 4), 16: Clausiliidae (sample 21), 17: Chondrula tridens (sample 4).

entwickelt sein. Zu hoffen bleibt, dass durch weiteres und besser erhaltenes Material eine gesicherte systematische $\mathrm{Zu}-$ ordnung der Form möglich wird.

Harmonierend mit den pollenanalytischen Befunden kann in dieser frühen Phase des Paläosees im Umfeld eine parkähnliche Landschaft rekonstruiert werden. Die vorgefundenen Gesellschaften verweisen insbesondere mit $\mathrm{Ce}$ paea sp., Truncatellina cylindrica, Vitrina pellucida, Vertigo pygmaea, Vitrina pellucida oder Vertigo pygmaea, Vertigo antivertigo und Vertigo angustior auf warmzeitliche Verhältnisse. Kaltzeitliche Leitarten fehlen vollständig.

Während die Proben 5 und 6 neben zahlreichen Individuen von Süßwasserschnecken noch Landschnecken, z. B. Vallonia costata, Pupilla muscorum, Truncatellina cylindrica und Vertigo pygmaea, führen, nehmen in den Proben 7-18 die Arten- und Individuenzahlen stark ab. Bei den Süßwasserarten bleibt jedoch die Dominanz von Bithynia tentacu-

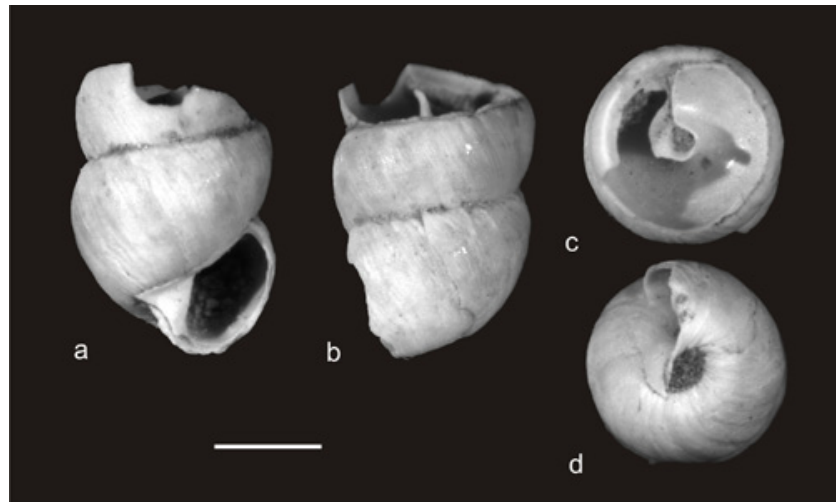

Taf. 2: Fragment einer Orculidae? (Probe 4, Hauptprofil A, Becken NN2). Plate 2: Fragment of a Orculidae? (sample 4, main profile A, basin NN2).

lata, Valvata piscinalis und Gyraulus laevis erhalten. Zudem konnte mit cf. Hydrobia sp. auch ein echter Salzanzeiger nachgewiesen werden (siehe unten). Die geringen Individuendichten machen es wahrscheinlich, dass die Art nicht im Seebecken selbst lebte, sondern dass ihr Vorkommen an Salzwasseraustritte bzw. Salzanreicherungen im weiteren Umfeld der Fundstelle gebunden war. Die kleinen Gehäuse dieser Gruppe können sehr leicht von Vögeln verschleppt oder durch sedimentologische Prozesse eingetragen werden. Nachgewiesen wurde die Art auch in dem archäologischen Hauptfundhorizont NN2/2 am nördlichen Beckenrand (5.3).

Von Landschnecken liegen z. B. mit Vallonia costata, Vallonia pulchella, Pupilla muscorum, Agriolimacidae/Limacidae oder Cepaea sp. nur noch ganz vereinzelte Nachweise vor.

Die durch Gips-Anreicherungen gekennzeichnet Schluffe im Bereich der Probe 16 (siehe oben) enthielten keine Molluskenreste.

In den Proben 19-23 erhöhen sich wieder die Arten- und Individuendichten deutlich. In der Süßwasserfauna sind zunächst, wie in den Liegendschichten, Bithynia tentaculata, Gyraulus laevis und Valvata piscinalis vorherrschend. Hinzu tritt jetzt erstmals Anisus leucostoma. Weitere Elemente sind Gyraulus crista, Radix cf. balthica und Lymnaea stagnalis. Auffällig ist, dass zum Hangenden, vor allem in den Proben 20-23, die Dominanzen von Bithynia tentaculata, Gyraulus laevis und Valvata piscinalis stark rückgängig sind und diese Arten durch Anisus leucostoma und Gyraulus crista hinsichtlich ihrer individuellen Häufigkeiten regelrecht ersetzt werden. Anisus leucostoma und Gyraulus crista bevorzugen kleine bzw. flache pflanzenreiche Gewässer. Diese Entwicklung ist als ein deutliches Anzeichen für die zunehmende Verflachung und Verlandung des Gewässers während des späten Eems zu werten. Gestützt wird diese Vermutung auch durch das im Profil erstmalige Auftreten der amphibisch lebenden und ebenfalls Kleingewässer bevorzugenden Sumpfschnecke Galba truncatula.

In der hellgrauen Kalkmudde (Pr. 19) fehlen Landschnecken vollständig. Sie fanden sich aber in geringen Individuendichten in den Proben 20-23. Nachgewiesen wurde ein Vertreter des Succinea/Oxyloma-Komplexes, ein Bewohner von nassen Gewässerufern oder Sümpfen. Relativ häufig ist auch wieder der Offenlandbewohner Pupilla muscorum vertreten. Von Schließmundschnecken (Clausiliidae), hauptsächlich Bewohner von Gehölzstandorten, liegen nur Fragmente vor, die keine nähere Zuordnung erlauben. 
Tab. 4a: Mollusken aus dem Hauptprofil A von 2007, Becken NN2.

Tab. 4a: Molluscs of the main profile A from 2007, basin NN2.

\begin{tabular}{|c|c|c|c|c|c|c|c|c|c|c|c|c|c|c|}
\hline Molluskenproben & U5/6 & 1 & 2 & 3 & 4 & 5 & 6 & 7 & 8 & 9 & 10 & 11 & 12 & 13 \\
\hline 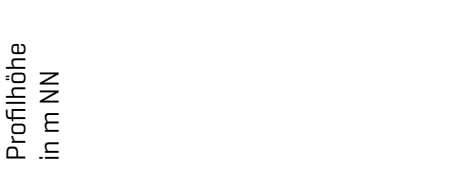 & 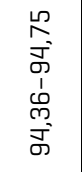 & 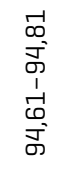 & 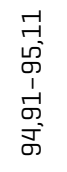 & 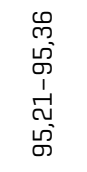 & 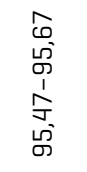 & $\begin{array}{l}\text { வ } \\
0 \\
0 \\
0 \\
1 \\
0 \\
\infty \\
\infty \\
0\end{array}$ & 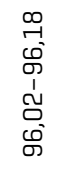 & 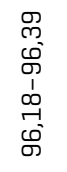 & 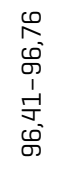 & 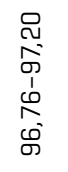 & 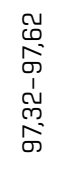 & $\begin{array}{l}\text { م } \\
\infty \\
\text { ஸ́ } \\
1 \\
0 \\
0 \\
\text { ள் }\end{array}$ & 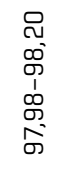 & $\begin{array}{l}10 \\
10 \\
\infty \\
\infty \\
1 \\
1 \\
\infty \\
m \\
\infty \\
\infty\end{array}$ \\
\hline \multicolumn{15}{|l|}{ Wasser } \\
\hline cf. Hydrobia sp. & - & - & - & - & - & - & - & - & - & 1 & - & - & - & - \\
\hline Bithynia tentaculata [LinnaEus 1758] & 3 & 41 & 3 & 425 & 887 & 92 & 6 & 1 & 8 & 5 & 8 & Frg. & - & 4 \\
\hline Bithynia laechii [SHEPPARD 1823] & - & - & - & - & - & - & - & - & - & - & - & - & - & - \\
\hline Valvata piscinalis [0. F. MüLleR 1774] & - & Frg. & - & 86 & 70 & 8 & 11 & - & 2 & - & 1 & - & - & Frg. \\
\hline Lymnaea stagnalis [LINNAEUs 1758] & - & - & - & - & - & - & - & - & - & - & - & - & - & - \\
\hline Stagnicola sp. & - & - & - & - & - & - & - & - & - & - & - & - & - & - \\
\hline Galba truncatula [0. F. MüLLER 1774] & - & - & - & - & - & - & - & - & - & - & - & - & - & - \\
\hline Radix cf. balthica [LINNAEus 1758] & - & - & - & 12 & 20 & - & 1 & - & 1 & - & - & - & - & - \\
\hline Radix sp. & - & - & - & - & - & 1 & - & - & - & - & - & - & - & - \\
\hline Myxas glutinosa [0. F. MüLler 1774] & - & - & - & - & - & - & - & - & - & - & - & - & - & - \\
\hline Lymnaeidae & Frg. & Frg. & Frg, & Frg. & Frg. & Frg. & Frg. & Frg. & Frg. & Frg. & Frg. & Frg. & Frg. & Frg. \\
\hline Planorbis planorbis [LINNAEUs 1758] & - & - & - & - & - & - & - & - & - & Frg. & - & - & - & - \\
\hline Anisus leucostoma [MILLet 1813] & - & - & - & - & - & - & - & 1 & - & - & - & - & - & - \\
\hline $\begin{array}{l}\text { Bathyomphalus contortus } \\
\text { [LINNAEUs 1758] }\end{array}$ & - & - & - & 2 & - & - & - & - & - & - & - & - & - & - \\
\hline Gyraulus laevis [ALDER 1838] & 1 & Frg. & - & 542 & 742 & 112 & 43 & 1 & 4 & 20 & 3 & - & - & Frg. \\
\hline Gyraulus crista [LINNAEUs 1758] & - & - & - & 30 & 78 & 3 & 8 & - & 1 & - & - & - & - & - \\
\hline Hippeutis complanatus [LINNAEUs 1758] & - & - & - & 3 & 2 & - & - & - & - & - & - & - & - & - \\
\hline Sphaerium corneum [LInNAEus 1758] & Frg. & Frg. & - & $2 \mathrm{~K}$ & Frg. & Frg. & Frg. & - & Frg. & Frg. & Frg. & Frg. & Frg. & - \\
\hline Pisidium subtruncatum MALM 1855 & - & - & - & $1 \mathrm{~K}$ & - & - & - & - & - & - & - & - & - & - \\
\hline Pisidium nitidum JenYns 1832 & - & - & - & - & $3 \mathrm{~K}$ & - & - & - & - & - & - & - & - & - \\
\hline Pisidium sp. & - & - & - & - & - & - & - & - & - & Frg. & - & - & - & - \\
\hline \multicolumn{15}{|l|}{ Land } \\
\hline Succinea/Oxyloma-Komplex & - & - & - & - & - & - & - & - & - & - & - & - & - & - \\
\hline Succinella oblonga [Draparnaud 1801] & - & - & - & - & - & - & - & - & - & - & - & - & - & - \\
\hline Cochlicopa lubrica [O. F. MülLeR 1774] & - & - & - & - & - & - & - & - & - & - & - & - & - & - \\
\hline Cochlicopa nitens [Gallenstein 1848] & - & - & - & - & - & - & - & - & - & - & - & - & - & - \\
\hline Cochlicopa sp. & - & - & - & 1 & - & - & - & - & - & - & - & - & - & - \\
\hline Truncatellina cylindrica [FÉRUSSAC 1807] & - & - & - & 3 & 15 & 3 & 1 & - & - & - & - & - & - & - \\
\hline Vertigo pygmaea [DraPARnaud 1801] & - & - & - & 12 & 33 & 2 & - & - & - & - & - & - & - & - \\
\hline Vertigo antivertigo [DRAPARnAud 1801] & - & - & - & - & 1 & - & - & - & - & - & - & - & - & - \\
\hline Vertigo angustior JefFREYS 1830 & - & - & - & 1 & - & - & - & - & - & - & - & - & - & - \\
\hline Vertigo sp. & - & - & - & - & - & - & - & - & - & - & - & - & - & - \\
\hline Orculidae? & - & - & - & - & 1 & - & - & - & - & - & - & - & - & - \\
\hline Pupilla muscorum [Linnaeus 1758] & - & - & - & 46 & 103 & 8 & - & - & - & - & 1 & - & - & - \\
\hline Pupilla triplicata [StUder 1820] & - & - & - & 1 & 2 & - & - & - & - & - & - & - & - & - \\
\hline Vallonia costata [0. F. MüLler 1774] & - & - & - & 379 & 719 & 54 & 12 & - & - & - & - & - & - & Frg. \\
\hline Vallonia pulchella [0. F. MüLLER 1774] & - & - & - & 14 & 39 & 4 & - & - & - & - & - & - & - & - \\
\hline Vallonia excentrica SteRKI 1892 & - & - & - & - & 5 & - & - & - & - & - & - & - & - & - \\
\hline Chondrula tridens [0. F. MüLLER 1774] & - & - & - & 14 & 12 & - & - & - & - & - & - & - & - & - \\
\hline Vitrina pellucida [0. F. MüLLER 1774] & - & - & - & - & 1 & - & - & - & - & - & - & - & - & - \\
\hline Nesovitrea hammonis [STRōM 1765] & - & - & - & 2 & 3 & - & - & - & - & - & - & - & - & - \\
\hline Agriolimacidae/Limacidae & - & - & - & 4 & 5 & 1 & - & - & - & - & - & - & - & - \\
\hline Clausiliidae & - & - & - & - & - & - & - & - & - & - & - & - & - & - \\
\hline Fruticicola fruticum [0. F. MülLeR 1774] & - & - & - & Frg. & Frg. & - & - & - & - & - & - & - & - & - \\
\hline Trichia hispida [LInNaEus 1758] & - & - & - & - & Frg. & - & - & - & - & - & - & - & - & - \\
\hline Euomphalia strigella [DRAPARnAud 1801] & - & - & - & Frg. & Frg. & - & - & - & - & - & - & - & - & - \\
\hline Arianta arbustorum [LINNAEUs 1758] & - & - & - & Frg. & Frg. & - & - & - & - & - & - & - & - & - \\
\hline Cepaea sp. & - & - & - & Frg. & Frg. & - & - & - & - & - & - & - & Frg. & - \\
\hline Helicidae & - & - & - & Frg. & Frg. & Frg. & - & - & - & - & - & - & - & - \\
\hline Artenzahl & 4 & 5 & 2 & 26 & 29 & 14 & 9 & 4 & 7 & 8 & 6 & 3 & 3 & 5 \\
\hline Individuenzahl & 6 & 45 & 4 & 1589 & 2749 & 291 & 84 & 4 & 18 & 41 & 15 & 3 & 3 & 8 \\
\hline
\end{tabular}


Tab. 4b: Mollusken aus dem Hauptprofil A von 2007, Becken NN2.

Tab. 4b: Molluscs of the main profile A from 2007, basin NN2.

\begin{tabular}{|c|c|c|c|c|c|c|c|c|c|c|c|c|}
\hline Molluskenproben & 14 & 15 & 16 & 17 & 18 & 19 & 20 & 21 & 22 & 23 & 24 & 25 \\
\hline 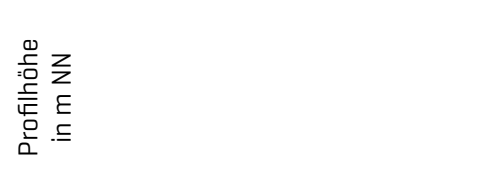 & \begin{tabular}{l}
$n$ \\
$\infty$ \\
$\infty$ \\
$\infty$ \\
$\sigma$ \\
1 \\
\multirow{1}{1}{} \\
$\infty$ \\
$\infty$
\end{tabular} & 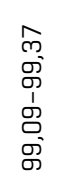 & 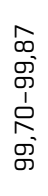 & 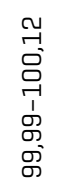 & 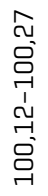 & 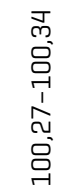 & 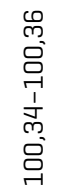 & 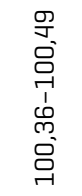 & 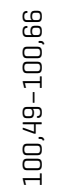 & 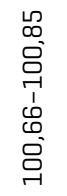 & 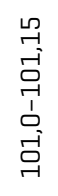 & 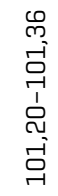 \\
\hline \multicolumn{13}{|l|}{ Wasser } \\
\hline cf. Hydrobia sp. & 1 & - & - & - & - & - & - & - & - & - & - & - \\
\hline Bithynia tentaculata [LINNAEus 1758] & 10 & 7 & - & - & 6 & 501 & 108 & 58 & 14 & - & - & - \\
\hline Bithynia leachii [SHEPPARD 1823] & - & - & - & - & - & 1 & - & - & - & - & - & - \\
\hline Valvata piscinalis [0. F. MüLLeR 1774] & - & - & - & - & - & 7 & 3 & - & - & - & - & - \\
\hline Lymnaea stagnalis [LINNAEUs 1758] & - & - & - & - & - & - & - & 25 & 1 & 11 & - & - \\
\hline Stagnicola sp. & - & - & - & - & - & - & 1 & - & 8 & - & - & - \\
\hline Galba truncatula [0. F. MüLleR 1774] & - & - & - & - & - & - & 2 & 12 & 4 & 4 & 2 & 8 \\
\hline Radix cf. balthica [LInNaEus 1758] & - & - & - & - & - & 34 & 17 & 131 & - & - & - & - \\
\hline Radix sp. & - & - & - & - & - & - & - & - & 18 & 17 & 1 & - \\
\hline Myxas glutinosa [0. F. MüLler 1774] & - & - & - & - & - & - & - & 1 & - & - & - & - \\
\hline Lymnaeidae & Frg. & Frg. & - & Frg. & - & Frg. & Frg. & Frg. & Frg. & Frg. & Frg. & Frg. \\
\hline Planorbis planorbis [LINNAEUs 1758] & - & - & - & - & - & - & Frg. & 5 & Frg. & - & - & - \\
\hline Anisus leucostoma [MILLET 1813] & - & 1 & - & - & - & 2 & 40 & 943 & 103 & 277 & 40 & 14 \\
\hline Bathyomphalus contortus [LINNAEUs 1758] & - & - & - & - & - & - & - & - & - & - & - & - \\
\hline Gyraulus laevis [ALdER 1838] & 5 & 3 & - & - & - & 787 & 130 & 149 & 44 & 12 & 3 & 7 \\
\hline Gyraulus crista [LINNAEus 1758] & - & - & - & - & - & 63 & 23 & 751 & 82 & 83 & 10 & - \\
\hline Hippeutis complanatus [LINNAEUs 1758] & 1 & - & - & - & - & 1 & - & - & - & - & - & - \\
\hline Sphaerium corneum [LInNAEus 1758] & - & Frg. & - & - & - & - & - & - & - & - & - & - \\
\hline Pisidium subtruncatum Malm 1855 & - & - & - & - & - & - & - & - & - & - & - & - \\
\hline Pisidium nitidum Jenyns 1832 & - & - & - & - & - & - & - & - & - & - & - & - \\
\hline Pisidium sp. & - & - & - & - & - & - & - & - & - & - & - & - \\
\hline \multicolumn{13}{|l|}{ Land } \\
\hline Succinea/Oxyloma-Komplex & - & - & - & - & - & - & 1 & 5 & - & 13 & 2 & 1 \\
\hline Succinella oblonga [Draparnaud 1801] & - & - & - & - & - & - & - & - & - & - & 8 & 17 \\
\hline Cochlicopa lubrica [O. F. MülLeR 1774] & - & - & - & - & - & - & - & - & - & - & - & 1 \\
\hline Cochlicopa nitens [GalLenstein 1848] & - & - & - & - & - & - & - & - & - & - & - & 1 \\
\hline Cochlicopa sp. & - & - & - & - & - & - & - & - & - & - & - & - \\
\hline Truncatellina cylindrica [FÉRUSSAC 1807] & - & - & - & - & - & - & - & - & - & - & - & - \\
\hline Vertigo pygmaea [Draparnaud 1801] & - & - & - & - & - & - & - & - & - & - & - & 1 \\
\hline Vertigo antivertigo [DRAPARNAUD 1801] & - & - & - & - & - & - & - & - & - & - & - & - \\
\hline Vertigo angustior JEFFREYS 1830 & - & - & - & - & - & - & - & - & - & - & 2 & - \\
\hline Vertigo sp. & - & - & - & - & - & - & - & Frg. & - & - & - & - \\
\hline Orculidae? & - & - & - & - & - & - & - & - & - & - & - & - \\
\hline Pupilla muscorum [Linnaeus 1758] & - & - & - & - & - & - & 2 & 2 & - & 6 & 19 & 75 \\
\hline Pupilla triplicata [StUder 1820] & - & - & - & - & - & - & - & - & - & - & - & - \\
\hline Vallonia costata [0. F. MüLleR 1774] & - & - & - & - & - & - & - & - & - & - & - & - \\
\hline Vallonia pulchella [0. F. MüLLER 1774] & 3 & 4 & - & - & - & - & - & 1 & - & - & - & 2 \\
\hline Vallonia excentrica STERK 1892 & - & - & - & - & - & - & - & - & - & - & 1 & 1 \\
\hline Chondrula tridens [0. F. Müller 1774] & - & - & - & - & - & - & - & - & - & - & - & - \\
\hline Vitrina pellucida [0. F. Müller 1774] & - & - & - & - & - & - & - & - & - & - & - & - \\
\hline Nesovitrea hammonis [STRöM 1765] & - & - & - & - & - & - & - & - & - & - & - & - \\
\hline Agriolimacidae/Limacidae & - & 2 & - & - & - & - & 2 & 5 & - & - & - & - \\
\hline Clausiliidae & - & - & - & - & - & - & - & Frg. & - & - & - & - \\
\hline Fruticicola fruticum [0. F. MüLleR 1774] & - & - & - & - & - & - & - & - & - & - & - & - \\
\hline Trichia hispida [LINNAEus 1758] & - & - & - & - & - & - & - & - & - & 1 & 1 & 3 \\
\hline Euomphalia strigella [DRAPARnAUd 1801] & - & - & - & - & - & - & - & - & - & - & - & - \\
\hline Arianta arbustorum [LINNAEUs 1758] & - & - & - & - & - & - & - & - & - & - & - & Frg. \\
\hline Cepaea sp. & - & - & - & - & - & - & - & - & - & - & - & - \\
\hline Helicidae & - & - & - & - & - & - & - & - & - & - & - & Frg. \\
\hline Artenzahl & 6 & 9 & $\mathbf{0}$ & 1 & 1 & 9 & 13 & 16 & 10 & 10 & 12 & 15 \\
\hline Individuenzahl & 21 & 21 & 0 & 1 & 6 & 1397 & 331 & 2091 & 276 & 425 & 90 & 135 \\
\hline
\end{tabular}


Die bräunlichen schluffigen Sande der Proben 24 und 25 repräsentieren pollenanalytisch bereits die frühe Weichselkaltzeit. In der Süßwasserfauna überwiegt jetzt Anisus leucostoma, was auf ein flaches Stillgewässer verweist. Die terrestrische Fauna ist vor allem durch die starke individuelle Zunahme von Offenlandelementen gekennzeichnet. Relativ häufig sind Feuchtlandbewohner, z. B. mit Succinea/Oxyloma, Cochlicopa nitens oder Vertigo angustior. Im Vergleich zur Eem-Basis kann deshalb von einer ausgedehnteren Sumpfvegetation ausgegangen werden, was ebenfalls mit der zunehmenden Verflachung des Seebeckens interpretiert werden kann. Größere individuelle Häufigkeiten erreichten Pupilla muscorum und Succinella oblonga. Diese Arten bevorzugen trockene bis mesophile, offene Habitate. In diesem Lebensraum finden sich z. B. auch Cochlicopa lubrica, Vertigo pygmaea, Vallonia excentrica und Trichia hispida. Ausgesprochene kaltzeitliche Leitarten fehlen. Diese konnten aber unmittelbar im Bereich des Hauptaufschlusses von 2007 bereits 2003 in Schluffen aus dem Profil B1 (Abb. 4: Pr. 20) mit Vallonia tenuilabris bestätigt werden.

\section{Das Hydrobia-Problem}

Die quartären Binnenland-Vorkommen von cf. Hydrobia sp. in Mitteldeutschland sind für Mitteleuropa einmalig (zusammenfassend MENG et al. 2004). Die Vorkommen nördlich des Thüringer Waldes sowie im Umfeld des Harzes (Thüringen und Sachsen-Anhalt, Abb. 1) sind an salzhaltige Grund- und Quellwasseraustritte gebunden, die vom Salinar des Zechsteins, des Röts oder des Mittleren $\mathrm{Mu}^{-}$ schelkalkes gespeist werden. Bekannt geworden sind vor allem die holozänen Massenvorkommen von cf. Hydrobia sp. im Gebiet der Mansfelder Seen bei Halle (z. B. CLEssin 1877, GoldFuss 1900). Weniger bekannt sind dagegen die zahlreichen Funde aus dem Pleistozän (zusammenfassend MEng et al. 2004). Die ältesten Nachweise von cf. Hydrobia sp. in Mitteldeutschland stammen aus dem altpleistozänen Tegelen-Komplex (Zeuchfeld bei Freyburg/Unstrut, Kalbsrieth). Weitere Funde sind bekannt aus dem unteren SaaleKomplex (Köchstedt/Salzmünde, Karsdorf, Krumpa, Hundisburg), aus der Eem-Warmzeit (Taubach, Neumark-Nord, Mansfelder Seen) sowie aus der Weichsel-Kaltzeit (Mansfelder Seen). Neben den bekannten holozänen Vorkommen der Mansfelder Seen fanden sich auch zahlreiche holozäne und subrezente Nachweise in der Umgebung von Artern und westlich von Magdeburg (Abb. 1).

Die Hydrobiidae innerhalb der Rissooidae gelten als äußerst schwer determinierbare Gruppe. Zudem existiert bis heute keine einheitliche Systematik. Eine sichere Bearbeitung ist nur mit Hilfe anatomischer sowie molekulargenetischer Methoden möglich. Die Bewertung gehäusemorphologischer Merkmale ist wegen der Ähnlichkeit der Gehäuse problematisch. Das Aussterbedatum von cf. Hydrobia sp. in Mitteldeutschland konnte bisher nicht konkret belegt werden. Aufgrund ihrer Vorkommen, z. B. in den Mansfelder Seen, muss man aber davon ausgehen, dass die Verbreitung der Art schon seit dem späten Mittelalter stark rückläufig war. Da in Mitteldeutschland aktuell keine Lebendvorkommen bekannt sind, können nur Gehäuse berücksichtigt werden. Bisher gelang deshalb keine gesicherte Zuordnung des vorliegenden Materials.

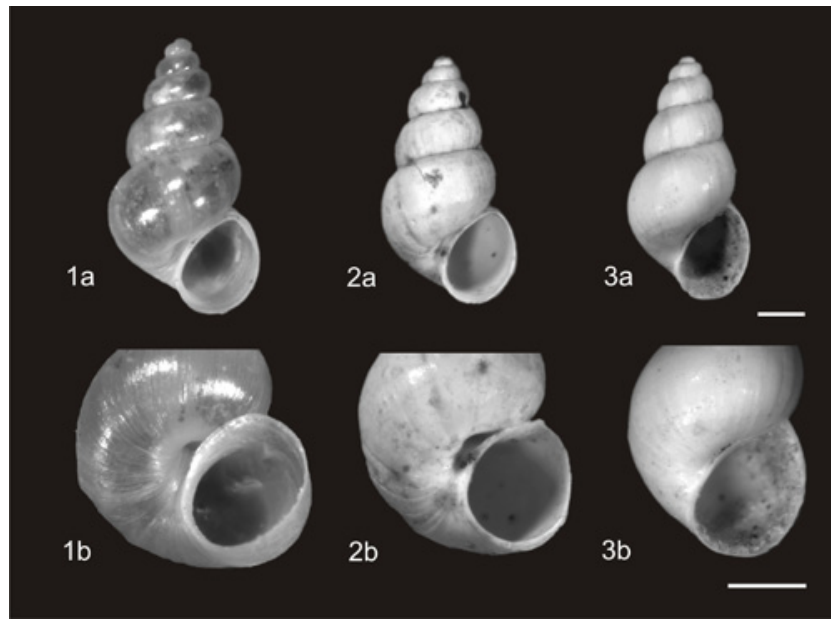

Taf. 3: Brackwasserschnecke cf. Hydrobia sp. aus Sachsen-Anhalt: 1. Mansfelder Seen, Holozän; 2. Köchstedt, Corbicula-Schotter des unteren SaaleKomplexes; 3. archäologischer Hauptfundhorizont NN2/2, Eem, NeumarkNord 2.

Plate 3: Brackish water snail cf. Hydrobia sp. from Saxony-Anhalt: 1. Mansfeld lakes, Holocene, 2. Köchstedt, Corbicula-gravels, Lower Saalian, 3. Main range of the archaeological findings NN2/2, Eemian, NN2.

Im klassischen Sinne handelt es sich bei den Vorkommen der Mansfelder Seen um Hydrobia stagnorum bzw. Hydrobia ventrosa, neu Ventrosia ventrosa (vgl. GöTTING 2008). Betrachtet man aber die Gehäuse verschiedener Vorkommen in Mitteldeutschland (Taf. 3), wird zudem deutlich, dass z. T. erhebliche morphologische Unterschiede, insbesondere bei der Gehäusebreite, der Wölbung der Umgänge sowie der Ausbildung des Nabels bestehen. Zu aller Problematik ist also auch davon auszugehen, dass in Mitteldeutschland von dieser Gruppe mehrere Arten vorkamen. Die Form von Neumark-Nord ist beispielsweise relativ schlank, die Umgänge sind weniger stark gewölbt und der Nabel ist lediglich als schwacher Schlitz ausgebildet.

\subsubsection{Kleinvertebraten [S. Meng]}

\section{Fische}

Häufig fanden sich im Profil auch isolierte Fischreste, beispielsweise in den Proben U 5/6, 3-5, 8 und 18-22. Bearbeitet wurden die Fische von Dr. G. Böhme, Berlin. Teilergebnisse liegen vor allem aus der fundreichen eemzeitlichen hellgrauen Kalkmudde (Pr. 19) vor. Die Fauna setzt sich aus Rotfeder (Scardinius erytrophthalmus), Karausche (Carassius carassius), Schleie (Tinca tinca), Flussbarsch (Perca fluviatilis) und Hecht (Esox lucius) zusammen. Der Flussbarsch konnte im Profil schon spätsaalezeitlich (Pr. U 5/6) belegt werden. Diese Fischgemeinschaft entspricht etwa der aus dem Becken NN1 bekannten Fauna (BöHmE 2010), welche typisch ist für Flachlandseen.

\section{Kleinsäuger}

Aus den Molluskenproben konnten nur wenige determinierbare Kleinsäugerreste geborgen werden. Es handelt sich um Molaren von Microtus arvalis - Feldmaus (Pr. 3) und Talpa sp. - Maulwurf (Pr. 4). Diese Arten unterstreichen ebenfalls den offeneren Charakter der Habitatstrukturen, besonders im Übergang Saale-Spätglazial/Eem. Die Kleinsäugerreste bearbeitete Dr. L. Maul, Weimar. 


\subsection{Lithologie der Profile B1, B2 und C [S. Wansa]}

Ergänzend zum Hauptprofil A sind weitere Detailaufnahmen in die Betrachtung einbezogen worden. Sie geben Aufschluss über die Differenziertheit der Ablagerungen im Becken NN2.

Bereits im Herbst 2003 bot sich im Profil B1, ca. $10 \mathrm{~m}$ südlich vom Hauptprofil A und ebenfalls im zentralen Beckenbereich gelegen, die Möglichkeit, den oberen Teil der Beckenfüllung zu dokumentieren, wobei die Profilsäule noch etwas weiter in das Hangende reicht als im Hauptprofil A (Abb. 6). Die Schichtenfolge ist der im Profil A ähnlich, und auch die Analysenergebnisse der Proben B1/15B1/22 stimmen, bis auf die in 4.3.1 erwähnte Diskrepanz beim Karbonatgehalt der Kalkmudde, im Wesentlichen mit denen aus Profil A überein.

Im Juni 2004 wurden im Profil B2, einem $3 \mathrm{~m}$ tiefen Schurf, der an das Profil B1 anschließt, die Schichten unmittelbar im Liegenden der markanten Mudde-Wechsellagerung untersucht (Abb. 13). Wie im Profil A treten auch hier starke Gipsausfällungen (überwiegend amorph, untergeordnet Kristallbildungen) verbunden mit Eisenoxidabsätzen auf Trennflächen und an Wurzelröhren auf. Die Proben 80-85 entsprechen etwa dem Profilabschnitt der Proben 12-16 in Profil A. Während die Schluffe in A zum Hangenden (bis Pr. 14) kontinuierlich feinkörniger werden und auch darüber noch Tongehalte um $30 \%$ aufweisen, variiert die Kornverteilung hier etwas stärker. Auffällig sind vor allem die grauweißen Grobschlufflinsen (Pr. 82), die wohl mit den horizontbeständigen Feinsandlinsen zwischen den Proben 12 und 13 im Profil A korrespondieren, sowie der dunkelbraungraue sehr stark tonige (46\%) Schluff von Probe 83. Die Kalkgehalte nehmen ähnlich wie in Profil A zum Hangenden ab (Pr. 82: $10 \%$, Pr. 85: 3 \%). Die Detailaufnahme (Abb. 13) zeigt auch, dass manche Schichten auskeilen oder an Verwerfungen bis ca. $20 \mathrm{~cm}$ (in einem Nachbaraufschluss bis $30 \mathrm{~cm}$ ) versetzt sein können.

Des Weiteren wurde im März 2007 das ca. 15 m nordwestlich von Hauptprofil A im Niveau von 97,5 bis 98,7 m NN gelegene Profil C dokumentiert. Hier standen an der nördlichen Beckenflanke geschichtete Schluffe an, die - vergleichbar dem Beckenzentrum (Profil A) - durch auffällige, an Straten gebundene Lagerungsstörungen (Fließtexturen) charakterisiert waren. Sowohl die Gefügeausbildung als auch die Analytik (Kornverteilung, Kalkgehalt) legen nahe, dass der hier aufgeschlossene Profilabschnitt dem ca. $2 \mathrm{~m}$ tiefer gelegenen Abschnitt der Proben 3-9 im Profil A entspricht. Danach fallen diese Schichten an der Beckenflanke zwischen den beiden Profilen mit 7-8 ${ }^{\circ}$ ein.

\subsection{Hangendschichten [S. Wansa]}

Die Seeablagerungen gehen im Hangenden in eine periglaziäre Schichtenfolge über, die zwar im Bereich des Hauptprofils A bis auf den Basisbereich abgetragen war, aber durch mehrere unweit davon erhaltene Teilprofile rekonstruiert werden konnte. Die Ergebnisse der Geländeaufnahmen von 2003 und 2004 und der Laboranalytik sollen hier nur synoptisch wiedergegeben werden. Für Detailinformationen sei auf LAURAT $\mho$ BRÜHL (2006), LAURAT et al. (2007) sowie MANiA (2010a) verwiesen.

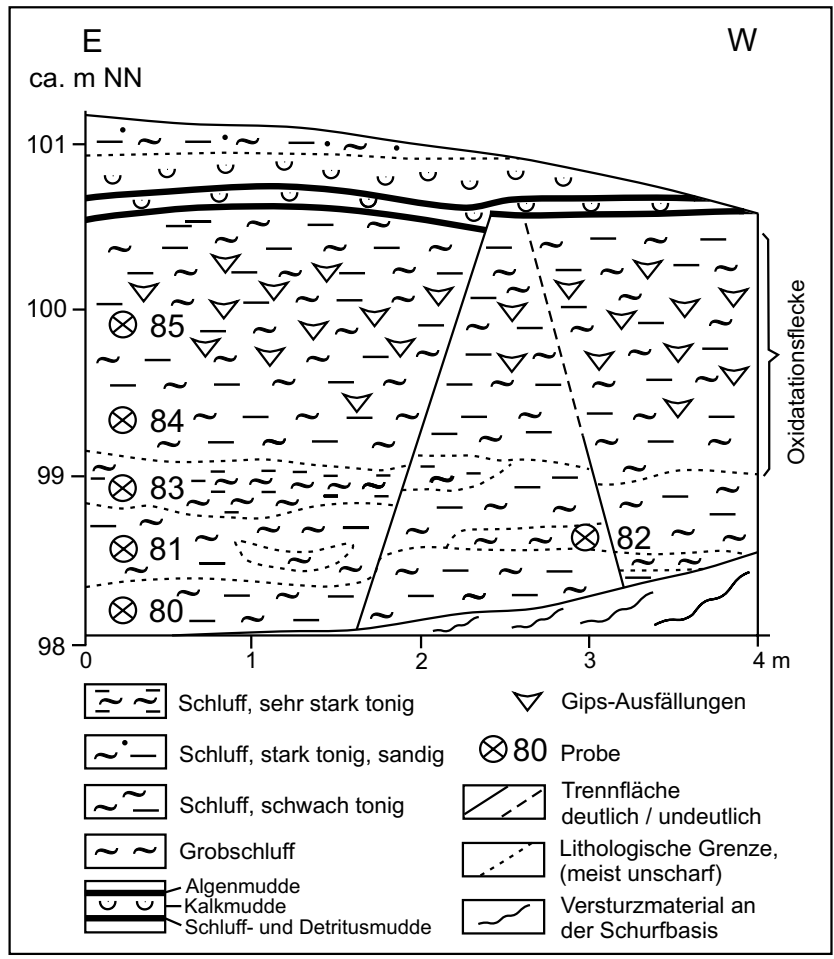

Abb. 13: Profil B2, Aufnahme Juni 2004.

Fig. 13: Profile B2, documentation fune 2004.

Der im Hauptprofil A nur partiell erhaltene Verzahnungsbereich von tonig-sandigem Seeschluff mit teils schwemmlössartigen Fließerden ist im Profil B1 ca. $70 \mathrm{~cm}$ mächtig (Abb. 6: Pr. B1/22, vgl. 4.3.1). Er wird hier durch eine helle, meist weniger als $10 \mathrm{~cm}$, max. $30 \mathrm{~cm}$ mächtige Sandschicht bedeckt, die auch wegen umfangreicher Artefaktfunde als Ufersand interpretiert wurde (archäologischer Fundhorizont NN2/0, LAURAT \& BRÜHL 2006). Im Hangenden schließt sich ein dunkles, nur wenige Zentimeter mächtiges kalkfreies Bodensediment an, das möglicherweise aus stark zersetztem Torf hervorgegangen ist (Abb. 6: Pr. B1/24). Darüber folgen wenige Dezimeter kalkfreier olivgrauer Schluff mit Sandlagen und ca. 0,5 m sandiger, fein- und grobkiesiger Mittelkies, der als fluviatiler Schotter angesehen wird und ebenfalls zahlreiche Artefakte enthält (Fundkomplex NN4). Die Geröllzusammensetzung weist mit mehr als $30 \%$ Kristallin und Feuerstein eine sehr hohe nordische Komponente auf, die nur durch Umlagerungen aus glaziärem Material der näheren Umgebung erklärbar ist. Weitere wesentliche Bestandteile des Geröllspektrums sind Quarz, Quarzit, Kalkstein und Porphyr. Das Spektrum der wenigen aus dem Ufersand gewonnenen Gerölle zeigt eine ähnliche petrographische Zusammensetzung.

Während der fluviatile Schotter im Gebiet von NN2 niveaubeständig (zwischen 102 und $103 \mathrm{~m} \mathrm{NN}$ ) verfolgbar ist, werden die liegenden weichselzeitlichen Sedimente sowie die eemzeitlichen Ufersedimente am nördlichen Beckenrand durch eine auffällige Diskordanz gekappt. Der Einschnitt erfolgte wahrscheinlich fluviatil, doch ist die entstandene Hohlform schnell durch Fließerden verfüllt worden. Diese enthalten Kiesnester mit einer der des übergreifenden Schotters nahezu identischen Geröllzusammensetzung. 
Unmittelbar nördlich des Beckens NN2 war das Hangende des Schotters aufgeschlossen. Die mehr als 5 m umfassende Sedimentfolge besteht in den unteren ca. $3 \mathrm{~m}$ aus grobschluffig-feinsandigen bis tonig-schluffigen Stillwasserablagerungen mit Fließerde- und Sandeinschaltungen und im oberen Teil aus Schwemmlöss und Löss. Der heterogen aufgebaute untere Teil weist horizontgebundene Oxidationsund Reduktionsmerkmale auf, die schwache hydromorphe Bodenbildungen anzeigen.

Im Niveau zwischen ca. 103 und 104 m NN waren nördlich des Beckens NN2 örtlich zwei auf Fließerden entwickelte humose Horizonte $\mathrm{zu}$ beobachten, die R. Ruske (freundl. mündl. Mitt.) dem Naumburger Bodenkomplex zugeordnet hat.

In den periglaziären Deckschichten treten horizontgebunden Froststrukturen in Erscheinung, meist in Form kleindimensionaler Kryoturbationen oder Eiskeile, die an Denudationsflächen ansetzen. Hervorgehoben seien die im hangenden Löss beobachteten bis ca. 2,5 $\mathrm{m}$ tiefen, meist schmalen, aber vielgestaltigen, im unteren Teil nur als Riss verfolgbaren Eiskeile.

\section{Das Becken NN2 im Vergleich mit dem Becken NN1}

\subsection{Sedimentationsbedingungen [S. Wansa, J. Luckert,} S. Meng \& J. Strahl]

Die Basis des Beckens NN2 bildet die Saale-Grundmoräne der Zeitz-Phase (4.2). Aufgrund ihrer Position in einer Randsenke zwischen Kohleaufragungen konnte sich über der Grundmoräne eine abflusslose Hohlform entwickeln, die in Anbetracht der nahezu vollständig nachgewiesenen saalespätglazialen und eemzeitlichen Sequenz über weit mehr als 10000 Jahre (Tab. 2) als Sedimentfalle diente. Unklar ist, in welchem exakten Zeitraum zwischen der ersten Saale-Vergletscherung und dem Ende des Saale-Komplexes die Randsenkenbildung erfolgte. Im Falle einer relativ frühzeitigen Anlage - bereits während der Vergletscherung - wäre davon auszugehen, dass die Hohlform bis zum Warthe-Stadium mit Toteis plombiert war, da die ersten palynologischen Belege in das Ende des Saale-Pleniglazials datieren (PZ A, Abb. 10).

Die Seeablagerungen des untersten Profilabschnittes $(90,4$ bis ca. 92,5 m NN) zeichnen sich durch ein breites Kornspektrum und geringe Sortierung aus, was darauf hindeutet, dass nach dem Beginn der Flachseebildung zunächst der umgebende saalezeitliche Geschiebemergel partiell abgetragen und als Umlagerungsprodukt am Seeboden resedimentiert wurde. Dafür sprechen auch die hohen Tonmineralgehalte bis in das Niveau von 92,0 m NN. Der allmähliche stete Anstieg der Quarzgehalte von < 30 Masse- $\%$ im untersten Meter der Beckenfüllung auf > 50 Masse-\%, verbunden mit einem deutlichen Anstieg des Schluffanteils sowie leicht erhöhten Kalkgehalten beruhen wahrscheinlich auf zunehmendem Eintrag äolischen Materials bzw. der Erosion von Lössablagerungen in der Umgebung. Diese Entwicklung setzt sich zum Hangenden fort, gefördert durch die bis weit in das Eem hinein offene Waldlandschaft, und wird erst durch die Zunahme des Tongehaltes ab ca. 98,5 m NN und die nachfolgende Bildung von Mudden unterbrochen. Mit dem Übergang zur Weichsel-Kaltzeit sind dann wieder höhere Grobschluff-Anteile zu verzeichnen. Die Annahme des differenzierten Eintrags äo- lischer Bildungen wird durch die Ergebnisse der palynologischen Untersuchungen gestützt (4.3.3).

Im Beckenzentrum sind die Sedimentationsbedingungen im Bereich von 92,5-96 m NN relativ konstant geblieben. Darüber lassen stratengebundene, synsedimentäre Fließgefüge auf eine relativ rasche Akkumulation und stärkeren lateralen Eintrag schließen. Dabei dürften sich die eng begrenzte räumliche Ausdehnung der Sedimentfalle sowie die relativ steilen Beckenflanken auf die gravitativen Bewegungen im Beckenzentrum begünstigend ausgewirkt haben. Molluskenanhäufungen verweisen in diesem Abschnitt auf ein verändertes Strömungsregime, wobei eine temporäre Sedimentzufuhr über ein Fließgewässer nicht ausgeschlossen werden kann. Sumpfarten fehlen hier fast vollständig.

Der gestörte Bereich (ca. 96-97 m NN) gehört größtenteils zur eemzeitlichen PZ 4, so dass eine Aktivierung des Kohlediapirismus als Ursache für die Schichtdeformationen wenig wahrscheinlich ist (vgl. ThOmAe 1990). Die außerdem beobachteten Zerrungsstrukturen in Form von kleindimensionalen Abschiebungen sind vermutlich durch Kompaktion und Setzung der Seeablagerungen entstanden.

Die zunehmende Kornverfeinerung zum Hangenden und das Abklingen der Lagerungsstörungen deuten auf eine zunächst ruhigere Sedimentation bei etwas erhöhtem Seespiegel hin. Dafür sprechen sowohl die palynologischen als auch die malakologischen Befunde hinsichtlich des Fehlens Flachwasser anzeigender Algen und Mollusken. Die im Bereich der PZ 5 bei ca. 98,5 m NN beobachtete ,gleyartige“ Nassbodenbildung (LAURAT et al. 2007), die darüber einsetzenden Gipsausblühungen bei gleichzeitiger Abnahme des Kalkgehaltes sowie die im Niveau von 99-100 m NN auftretenden Wurzelröhren zeigen nachfolgend eine Reduzierung der Seespiegelhöhe (vermutlich mit zeitweiligem Trockenfallen) an. Dies unterstreichen auch die palynologischen Belege verschiedener Jochalgen und die verstärkte Ausbreitung von Röhrichten bei insgesamt sehr schlechter Pollen- und Sporenkonservierung.

Die Kalkmudde zwischen den beiden organisch geprägten Lagen sowie die hangende molluskenreiche Kalkmudde (Abb. 6 und 9) repräsentieren Wiederbelebungsphasen der limnischen Sedimentation unter z. T. extremen Flachwasserbedingungen während der eemzeitlichen Schattholz- und abschließenden Lichtholzphase (PZ 7-9, Abb. 10), die mit dem Übergang in das Weichsel-Frühglazial durch Schluffe und Sande abgelöst wurden.

Aus den überlieferten Mächtigkeiten der die einzelnen Pollenzonen in Hauptprofil A repräsentierenden Sedimente und der Dauer der Pollenzonen (Tab. 2) lassen sich folgende Sedimentationsraten ableiten: PZ 4 (und PZ 3): 0,9 mm/a, PZ 5: 2,6 mm/a, PZ 6-9: 0,1 mm/a. Es handelt sich hierbei um Mittelwerte, die kaum Rückschlüsse auf die tatsächliche Dynamik des Sedimentationsgeschehens zulassen. Insbesondere der geringe Wert im Bereich der stärker organisch bzw. karbonatisch ausgebildeten Sedimente der PZ 6-9 dürfte durch Kompaktion beeinflusst sein.

Im Profil waren visuell keine Diskordanzen oder Schichtlücken erkennbar, die eine Mehrphasigkeit der Beckenbildung oder längere Sedimentationsruhe anzeigen würden. Dies wird durch die palynologischen Befunde soweit bestätigt, abgesehen von fehlenden Schichtgliedern des Saale-Spätglazials (PZ B, C 2) und der frühen Eem-Warmzeit 
(PZ 1), die entweder aufgearbeitet oder aufgrund zu geringer Sedimentationsraten bei der Beprobung nicht erfasst wurden.

Am nördlichen Beckenrand waren zeitweilig imposante staffelbruchartige Abschiebungen aufgeschlossen. Sie waren antithetisch orientiert und wiesen Versatzbeträge bis etwa $20 \mathrm{~cm}$ auf. Untergeordnet traten auch synthetische Verwerfungen sowie Grabenstrukturen auf. Diese Weitungstektonik, die in abgeschwächter Form auch im Beckenzentrum auftrat (4.4 und Abb. 13), resultiert wohl aus Setzungsvorgängen infolge der auflastbedingten Kompaktion von Seesedimenten im zentralen Beckenbereich. Allerdings kann eine späte (frühweichselzeitliche?) Wiederbelebungsphase des Kohlediapirismus als Ursache nicht völlig ausgeschlossen werden.

Das ehemals ca. $600 \times 400 \mathrm{~m}$ große, ovale, NW-SE orientierte Becken NN1 (MANIA 2004) war wohl um ein Vielfaches größer als das Becken NN2, auch wenn dessen ursprüngliche Gesamtfläche unbekannt ist (siehe 4.1). Die Basis beider, infolge Kohlediapirismus entstandener Hohlformen bildet die Erste Saale-Grundmoräne, über der noch unter kaltklimatischen Bedingungen die limnische Sedimentation einsetzte. Aus NN1 wurde eine bis ca. 3,5 m mächtige Sedimentfolge mit Bänderton, Schmelzwassersand und Beckenschluff beschrieben (MANIA 1990, 2004, MANIA et al. 2008), in NN2 sind es vor allem $5 \mathrm{~m}$ mächtige Seeschluffe (und -tone) die in Kornverteilung und mineralogischer Zusammensetzung eine markante Zweiteilung erkennen lassen. Die darüber lagernde warmzeitliche Sequenz ist in NN1 deutlich mächtiger als in NN2 und weist auch lithologische Unterschiede auf. Während in NN1 vorwiegend Detritusmudden, z. T. in Wechsellagerung mit anderen Muddearten und Torf akkumuliert wurden (zuletzt MANIA et al. 2008, 2010, Abb. 14), ist die Abfolge in NN2 klastischer geprägt. Hier wurden die Seeschluffe erst im jüngeren Eem (PZ 6-8) durch Detritus- und nachfolgend Kalk- und Algenmudde abgelöst. Dementsprechend unterschiedlich ist auch der Erhaltungszustand der Palynomorphen, der sich vor allem hinsichtlich der Nichterhaltung korrosionsanfälliger Sporomorphen, z. B. der Eibe und Esche, sehr nachteilig bei der palynologischen Interpretation auswirken kann.

Für die unterschiedlichen lithologischen Entwicklungen der Sedimentfüllungen der Becken NN1 und NN2 kommen mehrere Ursachen in Betracht. Von entscheidender Bedeutung dürfte die stark differierende Dimension der beiden Becken gewesen sein. Zudem sind die Flanken des Beckens NN2 zumindest örtlich deutlich steiler, wodurch die laterale Materialzufuhr begünstigt wurde. Außerdem muss mit Modifizierungen durch unterschiedliche Wassertiefen oder diachrone gravitative Bewegungen im Untergrund gerechnet werden. Das Niveau der zentralen Beckenbasis liegt wohl in NN2 um einige Meter höher als in NN1. In NN2 reicht die Grundmoränen-Oberfläche im Beckentiefsten nach geoelektrischen Messungen bis ca. $85 \mathrm{~m} \mathrm{NN}$ (Abb. 2 und 3, RAPPSILBER 2004b), in der Rammkernsondierung wurde sie bei 90,4 m NN festgestellt. Für die Basis der Beckensedimente in NN1 geben Thomae \& RAPpsiLber (2010) unter Bezugnahme auf eine Bohrung 81,4 m NN an.

Nach Mania (1990), Thomae (1990), Mania et al. (2008, 2010) und MANia (2010a) werden die interglazialen Seeablagerungen des Beckens NN1 von zwei jeweils 5-6 m

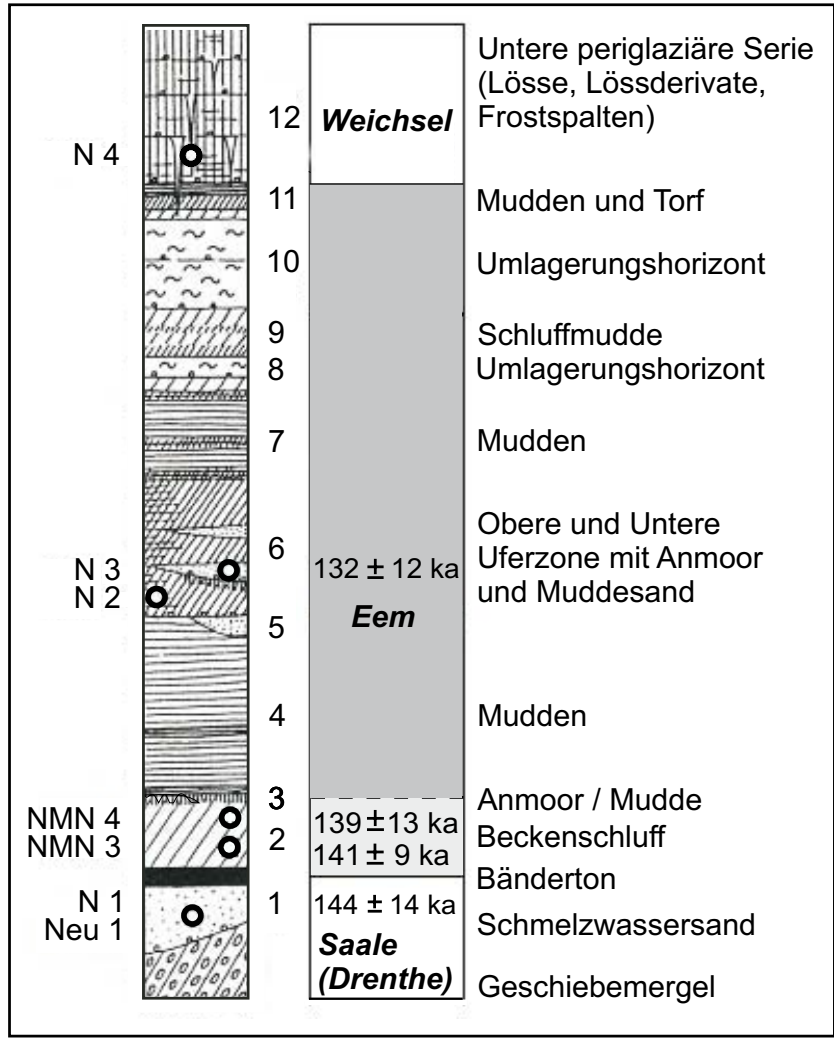

Abb. 14: Sedimentsequenz von Neumark-Nord 1 mit lithologischen Sedimentkomplexen 1 bis 12 (nach MANIA 2004, MANIA et al. 2010, Hangendbereich nicht vollständig abgebildet) und Ergebnissen von LumineszenzAltersbestimmungen (vgl. Tab. 6), Profilhöhe ca. $22 \mathrm{~m}$.

Fig. 14: Sediment sequence of Neumark-Nord 1 with lithological complexes 1-12 (after MANIA 2004, MANIA et al. 2010, top-section not shown), sample numbers and results of luminescence age determinations (see table 6), length of the profile about $22 \mathrm{~m}$.

mächtigen periglaziären Folgen überlagert, die im Wesentlichen aus Löss und Lössderivaten bestehen und Denudationshorizonte sowie Froststrukturen enthalten. Die beiden Folgen sind durch einen Bodenkomplex mit zwei Humusgleyen voneinander getrennt. Für MANIA (1990, 1994) stellt die Ausbildung der mächtigen Periglaziärsedimente ein gewichtiges stratigraphisches Argument dar, dem jedoch bereits von LiTT (1994b) plausibel widersprochen wurde. MANIA et al. (2008) verbinden die untere periglaziäre Folge von NN1 mit den basalen Sedimenten von NN2, womit die Diachronie der Becken NN1 und NN2 belegt werden soll. Diese Darstellung ist wegen der Charakteristik der saalezeitlichen Seeablagerungen in NN2 abzulehnen (siehe dieses Kap. sowie 4.3.1 und 4.3.3.1). Es ist vielmehr davon auszugehen, dass die periglaziären Deckschichten von NN1 ihre Entsprechung in den ebenfalls durch hydromorphe Bodenbildungen gegliederten, wenn auch geringer mächtigen Hangendschichten am nördlichen Rand des Beckens NN2 finden (4.5).

\subsection{Palynostratigraphie [J. Strahl]}

Ausgangspunkt der pollenstratigraphischen Diskussion ist die bis dato altersmäßig unterschiedliche Deutung der Sedimentfüllungen der Becken NN1 und NN2. Die in einer Vielzahl von Publikationen der Arbeitsgruppe von D. Mania angeführten genetischen, sedimentologischen und biostrati- 
graphischen Argumente mündeten letztendlich (MANIA et al. 2008, 2010) in einer Einstufung der interglazialen Ablagerungen hinsichtlich

- des Beckens NN1 in das Intra-Saale (sogenannte Neumark-Nord-Warmzeit inklusive dem unteren und oberen archäologischen Fundhorizont NN1, LAURAT et al. 2006),

- dem im Becken NN2 aufgefundenen archäologischen Hauptfundhorizont NN2/2 in eine darauffolgende weitere (!), palynostratigraphisch jedoch nicht definierte präwarthezeitliche Warmzeit und

- der darüber folgenden warmzeitlichen Ablagerungen inklusive des Fundkomplexes NN2/1 in das Eem-Interglazial.

Diesbezüglich wurden zum wiederholten Male (zuletzt Mania et al. 2010, Seifert-Eulen 2010) die nachfolgend $\mathrm{zu}$ diskutierenden palynostratigraphischen Argumente von SEIFERT (1990 und in MANIA 1992) zur Untermauerung eines intrasaalezeitlichen Alters des Interglazials von Neumark-Nord 1 in nahezu unveränderter Form zitiert. Den inzwischen vorliegenden Arbeiten von vor allem LitT (1994a, b), aber nachfolgend auch von KREMENETSKI (in Boettger et al. 2005, 2007), die diese Argumente nicht nur entkräfteten, sondern auch ein eemzeitliches Alter klar herausstellten, wurde dabei keine oder nur randlich Beachtung geschenkt. Die Behauptung von MANIA et al. (2010: 40), LitT (1994a) ignoriere die stratigraphischen Argumente, die einer Zuordnung zum Eem widersprächen, ist falsch, da in der angeführten Arbeit auf den S. 37-40 explizit darauf eingegangen wird. Zurückzuweisen sind auch folgende Ausführungen bezüglich der palynologischen Unterschiede zwischen Neumark-Nord und anderen Eem-Abfolgen in MANIA et al. (2010: 40): „Auffällig ist, dass diese Unterschiede von den Gegnern eines stärker mit Warmzeiten untergliederten Saale-Komplexes nicht angenommen und ignoriert werden.“ Die Untergliederung des glaziären Teiles des Saale-Komplexes durch Warmzeiten ist obsolet (siehe 1). Zudem werden die palynologischen Differenzen hier nochmals ausführlich diskutiert.

Ebenso ist die durch MANIA et al. (2010) vorgenommene Charakterisierung der Diagramme von LitT (1994a) und Kremenetski (in Boettger et al. 2007) als in der Abfolge der einzelnen Pollenklassen zu glatt und pauschal erscheinend, nicht zutreffend, wie nachfolgend aufgezeigt werden wird.

Nach SeIfERT (1990, in Mania 1992) bzw. ergänzt SEIfert-Eulen (2010) sowie MANia et al. (2008, 2010) sprechen folgende, in der Abb. 11b hervorgehobene, palynologische Argumente gegen eine Einstufung der Sedimentfüllung des Beckens NN1 in die Eem-Warmzeit:

1. die Maximal-Verbreitung der Fichte noch während der Hauptverbreitung der Hasel und vor der Hauptverbreitung der Hainbuche,

2. das Vorkommen der Tanne lediglich während der Hainbuchenphase und damit ein insgesamt anderes vegetationsgeschichtliches Verhalten von Hainbuche, Fichte und Tanne als in anderen Eem-Vorkommen,

3. die Maximalausbreitung der Eibe vor der Hasel,

4. die Maximalausbreitung der Ulme erst zu Beginn der Hainbuchen-Phase,
5. die nicht allmähliche Ausbreitung der Eiche, sondern ihr sehr unruhiger Kurvenverlauf, der in erneute Gipfel während der Hainbuchen-Phase mündet sowie insgesamt deutlich höhere Pollenfrequenzen als normalerweise im Eem,

6. der ständig hohe Anteil von Kräutern und Gräsern und

7. das Fehlen der Serbischen Fichte (Picea omoricoides) (von SEIFERT-EuLEN 2010 nicht mehr angeführt).

Die von SEIfERT bzw. SEIFERT-Eulen als grundsätzliche Abweichungen gegenüber der prinzipiellen eemzeitlichen Waldsukzession diskutierten besonderen, palynostratigraphisch gedeuteten Merkmale treffen, so sie tatsächlich vegetationsgeschichtlicher Natur sind, in der Zusammenschau (vgl. Abb. 11) auch auf das Becken NN2 zu. Ein Teil dieser Argumente geht jedoch ursächlich auf die Sedimentations- und Probennahmeumstände zurück.

Weitere, vor allem von SEIFERT-Eulen (2010) angeführte Gründe (Vorkommen des Tatarischen Ahorns, Ausbildung von Eichensteppenwäldern) berücksichtigen nicht ausreichend den durch seine zentrale Lage im Mitteldeutschen Trockengebiet bedingten klimatischen Sonderstatus des noch heute deutlich subkontinental geprägten Untersuchungsraumes. Sie können letztendlich in der komplexen Betrachtung nicht, wie schon durch LiTT (1994a, b) für das Becken NN1 eingehend erläutert, als triftige Gründe gegen ein eemzeitliches Alter angeführt werden. Randlich angemerkt sei, dass sowohl der Eutrophierungsgrad als auch Salzeinflüsse (beides auch für NN2 zutreffend!) keine Relevanz für die stratigraphische Position warmzeitlicher Ablagerungen besitzen (siehe rezente Salzstellen) und das Vorkommen NN1 nicht über der holsteinzeitlichen Körbisdorfer Terrasse (SEIFERTEulEn 2010: 271), sondern über der ersten Saale-Grundmoräne liegt. Zu den weiterhin von SEIFERT-Eulen (2010) als Beleg für ein höheres Alter als Eem angeführten TL-Altern siehe 6.1.

Bei der Bewertung dieser ausgewiesenen palynostratigraphischen Besonderheiten über den Vergleich der bisher publizierten Pollendiagramme, spielt die Ausbildung von Hiaten eine wesentliche Rolle. Zum einen sind dies tatsächliche Sedimentationslücken infolge der Einschaltung sandiger Ablagerungen bei ufernaher Profillage (Profil SEIFERT bzw. SEIFERT-Eulen in NN1) oder Erosion (Hauptprofil A in NN2). Zum anderen kann es sich aber auch um sekundäre, durch die gewählten Probenabstände verursachte Hiaten handeln. So umfasst keines der in Abb. 11 vergleichend vorgestellten Profile sowie auch das von KREMENETSKI (2010) präsentierte eine vollständige, vom ausgehenden SaaleGlazial kontinuierlich die Verhältnisse bis in das Weichsel-Frühglazial reflektierende Entwicklung. Sowohl dem NN1-Profil von Seifert (1990) bzw. Seifert-Eulen (2010, Abb. 11b) als auch dem von LiTt (1994a, Abb. 11c) bearbeiteten fehlen im Gegensatz zu dem primär als vollständig ausgewiesenen, tatsächlich jedoch erst im Klimaoptimum der Eem-Warmzeit (PZ 5 nach ERD 1973) wieder einsetzenden Profil von Kremenetski (in Boettger et al. 2005, 2007, Abb. 11d) saalespätglaziale Anteile. Weichselfrühglaziale Ablagerungen in NN1 wurden nur von SEIFERT (1990) bzw. SeIfert-Eulen (2010) erfasst, wohingegen das Profil von LiTT (1994a, b) noch innerhalb des Eems (PZ 7) abbricht. 
In NN2 wiesen das Hauptprofil A (Abb. 10 und 11a) und das Profil von KRemenetski (2010) insgesamt sehr gute Übereinstimmungen auf (vgl. 4.3.3). Aber auch sie sind durch Sedimentationslücken gekennzeichnet, die sowohl Abschnitte des Saale-Spätglazials (PZ B und C2 nach STrAhl \& HermsDORF 2008) als auch der Eem-Warmzeit (PZ 1 bzw. 1-4 unten nach ERD 1973) sowie des Weichsel-Frühglazials umfassen. Die von Kremenetski (2010) untersuchte Abfolge beinhaltet also nicht, wie von MANIA et al. (2010: 43) beschrieben, die gesamte Warmzeit.

Ebenfalls nicht zu vernachlässigen ist die Tatsache, dass lediglich das Profil von LitT (1994a, b) in NN1 sowie beide Profile in NN2 vertikal lückenlos entnommen wurden. Bei den übrigen Profilen handelt es sich um aus Teilprofilen zusammengesetzte Abfolgen, die, wie im Falle des Profils von KREMENETSKI aus NN1, versetzt über eine Erstreckung von $200 \mathrm{~m}$ im Aufschluss aufgenommen wurden. Unter Berücksichtigung dieser Fakten relativiert sich zumindest ein Teil der oben aufgeführten Argumente von SEIfERT bzw. SEIFERT-Eulen.

Eingeleitet wird die Entwicklung sowohl in NN1 (Abb. 11d) als auch in NN2 (Abb. 10 und 11a) durch stark mit präquartären und älterinterglazialen, vermutlich holstein- bis frühsaalezeitlichen Sporomorphen befrachteten Ablagerungen des Saale-Pleniglazials (PZ A), die in beiden Becken in die Akkumulation von Sedimenten des SaaleSpätglazials (PZ C1) mündeten. Neben der bereits für NN2 besprochenen Umdeutung der LPZ 1-3 von KREMENETSKI (2010) in die PZ A und C1, gilt dies aus den unter 4.3.3 diskutierten Gründen mit Sicherheit in NN1 (KREMENETSKI in BoetTGer ET AL. 2005, 2007) auch für die LPZ N1 (= PZ A) und zumindest den noch stark durch Umlagerungen behafteten Teil der LPZ N2 (= PZ C1). Auch das ermittelte IRSLAlter von $132 \pm 12 \mathrm{ka} \mathrm{BP}$ (vgl. Pr. N 3, Tab. 6) unterstreicht die stratigraphische Position des Profilabschnittes im Übergangsbereich Saale-Spätglazial/Eem. Die durch den Sanddorn (PZ B) bzw. durch die Kiefer (PZ C2) gekennzeichneten Abschnitte des Saale-Spätglazials sind dagegen in keinem der untersuchten Profile belegt.

Lediglich im Becken NN1 und wahrscheinlich nur im Profil von LiTT (1994a, b) ist die warmzeiteinleitende, früheemzeitliche Birken-Phase (PZ 1, Abb. 11c) erfasst. Alle übrigen Profile weisen einen zumindest bis in die PZ 2 (Abb. 11a und b) bzw. noch weiter in das Eem hineinreichenden, in Anbetracht der engen Probenabstände im Hauptprofil A (Abb. 10 und 11a) dort vermutlich echten $\mathrm{Hi}-$ atus auf (Abb. 11d und vgl. KREMENETSKi 2010). Nicht eindeutig lässt sich der obere, umlagerungsfreie Teil der LPZ N2 (Abb. 11d) des Profils von Kremenetski (in Boettger et al. 2005, 2007) in die saalespätglaziale PZ C1 bzw. in die eemzeitliche PZ 1 einordnen, da außer den auch durch LITT (1994a) ermittelten recht hohen Beifuß-Anteilen keine entsprechenden Spätglazialelemente, wie beispielsweise der Sanddorn, ausgewiesen sind. Lediglich mit der Ausbreitung von Rohr- und Igelkolben ist wie in NN2 ein Hinweis auf eine erste, saalespätglaziale Verlandungstendenz gegeben (vgl. 4.3.3.2).

Von Beginn an wird die interglaziale Waldentwicklung in beiden Becken durch sehr hohe Offenlandanteile (Abb.11ad, rote Einfassung der Totalsumme) begleitet, anfänglich insbesondere durch Beifuß und Süßgräser. Die Ursachen dafür liegen einerseits in den nachweislich im Becken NN1 bestehenden, stratigraphisch nicht relevanten und im übrigen auch in NN2 nachgewiesenen Salzwassereinflüssen (vgl. MAI 1992, 4.3.3.3), auf die LitT (1994a) vor allem die hohen Beifuß-Anteile zurückführt. Andererseits ist der in den vorangehenden Kapiteln bereits mehrfach angeführte, bis zum Beginn der Hainbuchen-Zeit bestehen bleibende parkartige Charakter der Bewaldung anzuführen, der ein langes, möglicherweise auch mittels der Beweidung durch Tiere gefördertes Überdauern lichtliebender Kräuter ermöglichte. Damit ist das von SEIFERT (1990) bzw. SEIFERT-Eulen (2010) unter Punkt 6 zitierte Argument hinfällig, zumal es bei der Einstufung des von KREMENETSKi (2010) aus NN2 publizierten Profils in das Eem durch MANIA et al. $(2008,2010)$ offensichtlich keine dahingehend einschränkende Rolle mehr spielte.

Zumindest Teile der Birken-Kiefern-Zeit (PZ 2), charakterisiert durch die Einwanderung der Ulme und nachfolgend der Eiche, reflektieren in NN1 die Diagramme von Seifert bzw. Seifert-Eulen (1990, 2010, Abb. 11b) und LitT (1994a, b, Abb. 11c) und des Hauptprofils A in NN2 (Abb. 10 und 11a). Sowohl in NN1 als auch NN2 besaß die Birke in diesem Zeitraum noch eine wesentliche Bedeutung, da lichtintensive Standorte weiterhin vorhanden waren. Eine endgültige Verdrängung setzte erst mit der Etablierung der Hasel ein.

Die Herausbildung kiefernreicher, thermophiler Laubmischwälder mit Ulme und Eiche in der Kiefern-Eichenmischwald-Zeit (PZ 3) belegt in NN1 am deutlichsten das Profil von Litt (1994a, b, Abb. 11c). Bei Seifert bzw. SeIfert-Eulen (Abb. 11b) ist lediglich ein Kulminieren der Eichen-Kurve erkennbar, das im Eem nach der Maximalverbreitung der Ulme liegt. Der nur mittels einer Probe dokumentierte Abschnitt der Waldentwicklung ist insbesondere auch angesichts des bei LiTT abgebildeten charakteristischen Ulmen-Maximums zu Beginn der PZ 3 (Abb. 11c) mit Sicherheit unvollständig erfasst. Dieser von SEIFERTEULEN (2010) dazu noch als außergewöhnlich hoch zitierte Eichenpollenwert von knapp 50 \% der Grundsumme ist, abgesehen davon, dass es sich um einen Einzelwert handelt, kein als „eemuntypisch“ belastbares Argument. Vergleichbare Prozentwerte finden sich häufig bei in Randposition befindlichen bzw. aus sehr kleinräumigen Sedimentationsbecken stammenden Profilen, so z. B. Straußberg und Borgisdorf (jeweils ca. $35 \%$, STRAHL \& HERMSDORF 2008), Klein Klütz Höved (45\%) und Hinterste Mühle (35\%). Nicht nur in NN1, sondern auch in NN2 ist während der Phase der Hainbuchen-Verbreitung (PZ 6-8a) eine nochmalige Wiederbelebung von Ulmen- (PZ 6) aber auch Eichenbeständen (PZ 7-8) reflektiert, wobei in NN2 die Ulme wegen ihrer besseren Erhaltungsfähigkeit gegenüber der Eiche deutlich in den Vordergrund tritt (Abb. 11a). Grundsätzlich zog sich die Ulme, wie vorhergehend bereits die Hasel und auch die Offenlandflora, aufgrund der zunehmenden Verschattung der Wälder bis zum Ende der Hainbuchen-Phase zurück. Lediglich die Eiche konnte sich, wie aus den Profilen in NN1 (Abb. 11b-d) zu ersehen, länger halten.

Somit greifen die Argumente 4 und 5 bezüglich einer fehlenden allmählichen Eichenausbreitung und einer Verlagerung des Ulmen-Maximums in den Beginn der Hainbuchen-Phase (PZ 6) als palynostratigraphisch relevante Merkmale ebenfalls nicht. Überdies bildet sich der „un- 
ruhige" Verlauf (nicht nur) der Eichen-Kurve auch ganz deutlich in den Profilen von NN2 und zwar insbesondere innerhalb der PZ 4 im Hauptprofil A mit scheinbar hierher verlagerten Maxima bei Eiche und Ulme ab (Abb. 10 und 11a, grüne Umrandung der Kurven). Während für das SEIfERT'sche Profil ursächlich seine Randposition anzuführen ist, sind hier die überwiegend schlechten Erhaltungsbedingungen bzw. die Sedimentationsumstände (teilweise Zusammenspülung) für den unruhigen Diagrammverlauf verantwortlich zu machen, also ebenfalls keine vegetationsgeschichtlichen Faktoren.

Im Unterschied $\mathrm{zu}$ den Profilen aus NN2 (Abb. 11a, Kremenetski 2010: 275) und dem LitT'schen Profil aus NN1 (Abb. 11c) ist der Abschnitt der haselreichen Eichenmischwald-Zeit (PZ 4) in den Profilen von SEIFERT (Abb. 11b) und Kremenetski (Abb. 11d) nicht vollständig bzw. gar nicht (Hiatus) überliefert. Somit ist der dem eemzeitlichen Eiben-Maximum vorangehende Verlauf der Hasel-Kurve einschließlich eventueller Maxima nur lückenhaft bzw. überhaupt nicht nachvollziehbar. Die indirekte Beweisführung von SEIFERT-EuLEN (2010: 271), das von KREMENETSKI (in Boettger et al. 2007) untersuchte Profil könne nicht eemzeitlich sein, weil das vor dem Hasel-Maximum liegende Eiben-Maximum nur aufgrund des dort ausgebildeten Hiatus fehle, ist eine reine Mutmaßung. Das diese nicht zutrifft, hätte bei Kenntnisnahme des Profils von LiTt und der daraufhin notwendigen Neubewertung ihres eigenen Profils klar werden müssen. Das dritte angeführte Argument, dass die Eibe in Neumark-Nord ihre Maximalverbreitung vor der Hasel erreicht hätte, ist somit gleichfalls palynostratigraphisch gegenstandslos.

Zum u. a. mehrfach angeführten Tatarischen Ahorn als eemuntypisches subkontinentales Steppenwaldelement sei der korrigierende Hinweis erlaubt, dass die Funde aus dem Becken NN1 keinesfalls erstmaligen Nachweischarakter hinsichtlich eines subkontinental geprägten Klimas im Elbe-Saale-Gebiet besitzen (vgl. MANIA et al. 2008), sondern auch für das eemzeitliche Becken von Grabschütz (LITT 1990) vorliegen. Wie für NN1 existieren für das Becken NN2 entsprechende frühe palynologische Ahorn-Nachweise, die jedoch nicht makrorestanalytisch unterlegt werden konnten. Des Weiteren sei an dieser Stelle darauf verwiesen, dass das Mitteldeutsche Trockengebiet bis heute seinen klimatischen Sonderstatus hinsichtlich seiner Niederschlagsarmut und seines subkontinentalen Einschlags beibehalten hat und daher noch heute Verbreitungsgebiet südlicher Arten ist (vgl. u. a. Meusel 1970, Benkert et al. 1996). So finden sich hier beispielsweise unter den $27, \mathrm{zu}^{-}$ letzt bei MANIA et al. (2010) genannten Arten mit südosteuropäisch-westasiatischem, südeuropäischem bzw. submediterranem Verbreitungsschwerpunkt 20, wovon 13 sogar weit über das Mitteldeutsche Trockengebiet hinaus vorkommen. Als enger auf diesen Raum beschränkt bleibend seien als Beispiele kontinental bis subkontinental geprägter Arten die Sand-Esparsette (Onobrychis arenaria), die Pferde-Sesel (Seseli hippomarathrum), die Acker-Spatzenzunge (Thymelaea passerina) sowie subtemperater bis temperater Arten der Wollige Schneeball (Viburnum lantana), der Stink-Gänsefuß (Chenopodium vulvaria) und der GemüsePortulak (Portulaca oleracea) genannt.

Die im Profil von SeIfert (Abb. 11b) im Vergleich zu den übrigen und für Neumark-Nord angesichts seiner besonderen Lage im Mitteldeutschen Trockengebiet sehr hoch ausfallenden Pollenfrequenzen der Eibe in der PZ 5 (Hasel-EibenLinden-Zeit) sind auf die Art und Weise der laborativen und in diesem Falle effektiveren Aufbereitung der Proben zurückzuführen. Eine gleichartige Anreicherung wurde auch für eine ganze Reihe von Eem-Profilen aus dem Brandenburger Raum festgestellt, die labortechnisch alle nach dem gleichen Modus vorbereitet wurden (vgl. dazu STRAHL \& HERMSDORF 2008: 38). Insgesamt zeigen die Profile in NN1 aufgrund der durchgehend besseren Erhaltung durchschnittlich höhere Eiben- und auch Eschen-Werte als in NN2. Einen übereinstimmenden Trend weisen aber alle Profile auf: Generell sind die Erle (ab der PZ 4) und die Linde (ab der PZ 5) innerhalb der verschiedenen eemzeitlichen Waldgemeinschaften unterrepräsentiert.

Entgegen den Untersuchungen von LitT (1994a, Abb. 11c) wird die bereits von SEIFERT (1990, Abb. 11b) beobachtete frühe Ausbreitung der Fichte im Übergang von der Phase der thermophilen Laubmischwaldgesellschaften zu den späteemzeitlichen Schattholzwäldern in der PZ 6 (HainbuchenZeit) sowohl in den Diagrammen aus NN1 (Abb. 11b und d) als auch NN2 (Abb. 11a und Kremenetski 2010) deutlich. Auf den mit großer Wahrscheinlichkeit durch Fernflug verursachten Eintrag des Fichtenpollens im Gegensatz zur späteren auch standörtlichen Förderung auf staunassen Arealen wurde bereits unter 4.3.3.3 eingegangen. Damit ist dieses besondere Verhalten, wie schon für die in beiden Becken mit hohen Anteilen festgestellten Offenlandelemente als palynostratigraphisches Argument gegen ein eemzeitliches Alter nicht haltbar.

Die für die PZ 7 (Hainbuchen-Fichten-Zeit) charakteristischen Züge der Waldentwicklung mit der Ausbreitung von Fichte und Tanne innerhalb der bis in die PZ 8 (KiefernFichten-Tannen-Zeit) hinein weiterhin sehr stark Hainbuchen-dominierten Bewaldung bleiben in allen Diagrammen mehr oder weniger undeutlich. So sind schon ab der PZ 6 die Sedimentationsraten in NN2 infolge des sich extrem verflachenden Sedimentationsraums sehr gering. Sie bedingen im Gegensatz zu NN1 (Abb. 11d) eine verzerrte bzw. sehr verkürzte Abbildung der Etablierung der Nadelgehölze. Letzteres gilt auch für das beckenrandlich situierte Profil von SeIfert (1990, Abb. 11b), wo Tannen- und Hainbuchenausbreitung während der PZ 7 und 8 scheinbar ineinander „verschwimmen“. Aufgrund der sehr eng gewählten Probenabstände in NN2 (Hauptprofil A, Abb. 11a) konnte zumindest ein Maximalausschlag der Tannen-Kurve (auch im Profil KREMENETSKi 2010) innerhalb der extrem geringmächtig ausgebildeten PZ 8 erfasst werden. Sowohl für NN1 als auch NN2 liegen zeitgleich hohe Hainbuchen- und Tannen-Werte zu Beginn der PZ 8 vor, die anscheinend typisch für das Mitteldeutsche Trockengebiet sind, da diese Situation auch von den Profilen Gröbern und Grabschütz (LiTt 1990, 1994a) reflektiert wird.

Klimatisch bedingt werden dann zunächst die Hainbuche und nachfolgend die Tanne in beiden Becken, wie für das Eem typisch, mit der erneuten Ausbreitung der Kiefer während der Kiefern-Zeit (PZ 9) aus den Wäldern verdrängt. Die Verhältnisse während der späteemzeitlichen Vegetationsentwicklung reflektiert dabei in NN1 besonders ausführlich das Diagramm des Profils von Kremenetski (in Boett- 
GER et al. 2005, 2007) mit dem Vordringen der Birke in die instabiler werdenden borealen Waldgemeinschaften. Somit ist, wie bereits durch LiTT (1994a, b) angeführt, auch dem Argument 2 von SEIFERT zu widersprechen, dass abgesehen von dem scheinbar ungewöhnlichen und oben diskutierten Verhalten der Fichte vor der Hainbuchenausbreitung (Argument 1) Abweichungen gegenüber der eemzeitlichen Vegetationsentwicklung bestünden.

In jedem Fall nicht nachzuvollziehen war das inzwischen von SeIFERT-EulEN (2010) nicht mehr angeführte Argument 7, da die Serbische Fichte, soweit bisher bekannt, ihre wesentliche Verbreitung in den Interstadialen des WeichselFrühglazials hatte und in den vorhergehenden Warmzeiten keine Rolle spielte.

Die Zusammenschau der warmzeitlichen Vegetationsentwicklung in beiden Becken zeigt also entgegen den Ausführungen von MANIA (zuletzt in MANIA et al. 2010) aus palynologischer Sicht eine durchgängige Übereinstimmung. Trotz der oben genannten einschränkenden bzw. beeinflussenden sedimentologischen und probennahmebedingten Faktoren sind in den vorliegenden Pollendiagrammen (Abb. 11a-d) deutlich die Grundzüge der eemzeitlichen Waldsukzession, und zwar die zeitlich versetzte Einwanderung von Birke - Kiefer - Ulme - Eiche - Hasel - Erle - Eibe - Linde - Hainbuche - Fichte - Tanne und erneut Kiefer und/oder Birke erkennbar. Beide Becken zeigen anhand der Zugrundelegung aller diskutierten Profile eine quasi kontinuierlich zu verfolgende Vegetationsentwicklung vom Saale-Spätglazial bis in die ausgehende Eem-Warmzeit. Das Weichsel-Frühglazial ist nur reliktisch erfasst.

Tatsächliche Besonderheiten der hiesigen eemzeitlichen Vegetationsentwicklung - und dies in beiden Becken - sind:

1. hohe Kräuterpollenanteile, die auf die Existenz einer parkartigen Bewaldung (Hasel, Eiche, Ulme) unter warmen, deutlich subkontinental getönten Klimabedingungen vor der Hainbuchenausbreitung schließen lassen,

2. nur geringe Erlen- und Lindenanteile und

3. eine nur scheinbar frühe Fichtenausbreitung, die in Verbindung mit in der Nähe liegenden Mittelgebirgsstandorten (Fernflugeintrag in Verbindung mit erhöhten Kräuter- und auch Kiefern-Werten) steht.

Sie ergeben sich hier vor allem aus der speziellen klimatischen Situation infolge der Lage der Aufschlüsse im zentralen Mitteldeutschen Trockengebiet. Wurde schon durch die Untersuchungen von LitT (1994a, b) und nachfolgend Kremenetski (in Boettger et al. 2005, 2007) für das Becken NN1 deutlich, dass die durch MANIA angeführten Argumente von SEIFERT (1990) hinsichtlich eines höheren Alters von NN1 nicht stichhaltig sein können, so wurde dies spätestens mit dem durch KREMENETSKI (2010) bearbeiteten Profil aus NN2 Gewissheit. Dieses von MANIA et al. (2010) als eemzeitlich akzeptierte Profil weist insbesondere hinsichtlich der Argumente 1 und 6 auch solche Merkmale auf, die nach Meinung derselben Autoren gegen das eemzeitliche Alter von NN1 sprechen sollen - eine Erklärung dafür wird nicht gegeben.

\subsection{Malakologische Interpretation [S. Meng]}

Die Süßwasserfauna zeigt eine einheitliche Entwicklung des Gewässers innerhalb eines warmzeitlichen Zyklus an, u. a. mit der Dominanz von Bithynia tentaculata, Gyraulus laevis und Valvata piscinalis. Zwar nimmt die individuelle Häufigkeit der Mollusken, besonders im mittleren Profilteil (vor allem Pr. 7-18) stark ab, für zwischengeschaltete kaltzeitliche Phasen gibt es aber keinerlei Indizien. Der obere Profilteil (Pr. 19-25) ist durch die starke individuelle Zunahme von Anisus leucostoma gekennzeichnet, was die zunehmende Verlandung des Gewässers ankündigt. Die terrestrische Molluskenfauna ist entsprechend dem limnischen Milieu weniger häufig. Sie wird zwar hauptsächlich durch Offenlandelemente, z. B. Pupilla muscorum und Vallonia costata vertreten, enthält aber auch eindeutige warmzeitliche Elemente, insbesondere mit Cepaea sp.

Die Molluskenfauna ist durch einige Besonderheiten charakterisiert. Auffällig ist, dass zahlreiche eemtypische Elemente in NN2 zu fehlen scheinen. Hierzu gehört beispielsweise die Quellschnecke (Belgrandia germanica), die in mitteldeutschen Eem-Vorkommen, besonders in Travertinen, in der Regel massenhaft entwickelt ist. Vermutlich fehlten im weiteren Umfeld der Fundstelle geeignete Quellhabitate. Anspruchsvolle thermophil geprägte Waldarten oder Exoten sind offenbar selten. Ausgehend von den geringen Individuenmengen können allerdings auch taphonomische Probleme diskutiert werden. Insgesamt muss aber von einer stärker kontinentalen Prägung der Faunen ausgegangen werden, was mit der Lage des Paläosees innerhalb des Mitteldeutschen Trockengebietes korreliert (vgl. 5.2).

Der archäologische Hauptfundfundhorizont NN2/2 war am nördlichen Beckenrand wenige Meter östlich des Profils D (Abb. 5) im Hangenden saalezeitlicher Schluffe aufgeschlossen. Der Fundhorizont wird hier durch helle Feinsande gebildet und ist etwa 0,5-1 m mächtig. Dem Vergleich mit dem Hauptprofil A liegen Probennahmen von S. MENG von 2005 sowie von S. WANSA von 2004 zu Grunde (Tab. 5). Mit der für NN2 insgesamt charakteristischen Häufigkeit von Bithynia tentaculata, Valvata piscinalis und Gyraulus laevis, fällt zudem auch das Fehlen von Anisus leucostoma in NN2/2 auf. Diese Art fehlt auch im unteren und mittleren Bereich des Hauptprofils A weitgehend, wird jedoch im oberen Abschnitt dominant (Abb. 12).

Insgesamt sind die Individuendichten im Bereich von NN2/2 am Beckenrand deutlich höher entwickelt, trotzdem sind die Diversitäten gut vergleichbar. Weitere charakteristische Elemente der Süßwasserfauna sind z. B. Radix cf. balthica, Radix auricularia, Myxas glutinosa sowie der Salzanzeiger cf. Hydrobia sp.

Die terrestrische Fauna ist hier ebenso wie im Beckenzentrum unterrepräsentiert. Relativ häufig sind die Offenlandelemente Pupilla muscorum und Vallonia costata. Nachgewiesen wurden außerdem das Steppenelement Chondrula tridens und die Warmzeitform Cepaea sp. Mit Vertigo antivertigo und Oxyloma sp. sind zudem auch vereinzelt feuchtigkeitsliebende Arten, Bewohner von nassen Gewässerufern oder Sümpfen, belegt. MANIA (in LAURAT ひ BRÜHL 2006, MANIA et al. 2008) erwähnt zudem noch die gehölzliebenden Elemente Aegopis verticillus, Cochlodina laminata oder Clausilia pumila. 
Tab. 5: Mollusken aus dem archäologischen Hauptfundhorizont NN2/2, Becken NN2.

Tab. 5: Molluscs of the main range of the archaeological findings NN2/2, basin NN2.

\begin{tabular}{|c|c|c|c|}
\hline Probennahmen & $\begin{array}{c}\text { Wansa } \\
13.05 .04\end{array}$ & $\begin{array}{c}\text { Wansa } \\
28.06 .04\end{array}$ & $\begin{array}{c}\text { Meng/Laurat } \\
30.06 .05\end{array}$ \\
\hline \multicolumn{4}{|l|}{ Wasser } \\
\hline cf. Hydrobia sp. & - & - & 3 \\
\hline Bithynia tentaculata [LINNAEUs 1758] & 250 & 645 & 3664 \\
\hline Valvata piscinalis [0. F. MüLler 1774] & 7 & 32 & 285 \\
\hline Stagnicola sp. & 3 & 4 & 3 \\
\hline Galba truncatula [0. F. MüLLER 1774] & - & - & 4 \\
\hline Radix cf. balthica [LInNAEus 1758] & 3 & 4 & 27 \\
\hline Radix auricularia [LINNAEUs 1758] & - & - & 3 \\
\hline Myxas glutinosa [0. F. MüLler 1774] & - & - & 6 \\
\hline Lymnaeidae & Frg. & Frg. & Frg. \\
\hline Gyraulus laevis [ALDER 1838] & 130 & 24 & 617 \\
\hline Gyraulus crista [LinnAeus 1758] & 6 & - & 11 \\
\hline Unionidae & Frg. & - & - \\
\hline Sphaerium corneum [LINNAEus 1758] & $1 \mathrm{~K}$ & $1 \mathrm{~K}$ & Frg. \\
\hline Pisidium spp. & - & - & $12 \mathrm{~K}$ \\
\hline \multicolumn{4}{|l|}{ Land } \\
\hline Oxyloma sp. & - & - & 3 \\
\hline Vertigo antivertigo [DRAPARnAUd 1801] & - & - & 1 \\
\hline Vertigo pygmaea [Draparnaud 1801] & 1 & - & - \\
\hline Pupilla muscorum [Linnaeus 1758] & - & - & 10 \\
\hline Vallonia costata [0. F. MüLleR 1774] & 33 & - & 3 \\
\hline Chondrula tridens [0. F. MüLLER 1774] & - & - & 1 \\
\hline Agriolimacidae/Limacidae & 1 & - & 1 \\
\hline Trichia sp. & 1 & - & - \\
\hline Fruticicola fruticum [0. F. MüLLER 1774] & 1 & - & Frg. \\
\hline Euomphalia strigella [DRAPARnAUd 1801] & 1 & - & - \\
\hline Cepaea sp. & - & Frg. & Frg. \\
\hline Helicidae & Frg. & - & Frg. \\
\hline Anzahl Proben & 4 & 2 & 4 \\
\hline Artenzahl & 16 & 8 & 22 \\
\hline Individuenzahl & 441 & 714 & 4669 \\
\hline
\end{tabular}

Die Molluskenfaunen des Seebecken NN1 ähneln, nach den Ergebnissen von MANIA (2000) zu urteilen, prinzipiell den Faunen von NN2 (Tab. 4 und 5). Zu bemerken ist aber auch, dass NN1 wesentlich besser aufgeschlossen war und intensiver malakologisch untersucht wurde. Der limnische Bereich wird beispielsweise von Bithynia tentaculata, Valvata piscinalis, Gyraulus laevis, Gyraulus crista, Anisus leucostoma u. a. charakterisiert. Ebenfalls nachgewiesen wurde in NN1 die Brackwasserschnecke cf. Hydrobia sp. Ausgesprochen ähnlich sind auch die Fischfaunen, mit Rotfeder, Schleie, Karausche, Flussbarsch und Hecht.

Sehr auffällig ist, dass im Bereich von NN1 auch bei den Landschnecken, vergleichbar mit NN2, eine relativ hohe individuelle Dominanz von Offenlandarten, ebenfalls mit $\mathrm{Pu}$ pilla muscorum, Vallonia costata, Vallonia pulchella, Truncatellina cylindrica, Vertigo pygmaea, Chondrula tridens u. a. vorliegt. Im Vergleich zu NN2 sind in NN1 mehr Arten von anspruchsvolleren Waldarten nachgewiesen. Sie erreichen aber offenbar ebenfalls nur geringe Häufigkeiten. Insgesamt ist also davon auszugehen, dass sich auch im Umfeld des Seebeckens NN1 parkähnliche Landschaften entwickelten und dass aufgrund der Ähnlichkeiten der Faunen beide Seebecken zur gleichen Zeit existierten.

\section{Geochronologie}

\subsection{Lumineneszenzdatierungen an Sedimenten der Becken NN1 und NN2 [M. Krbetschek \& L. Zöller]}

\subsubsection{Diskussion der Daten von Karelin [1997]}

Bereits in den 1990er Jahren wurden TL-Datierungen an Sedimenten des Beckens NN1 innerhalb eines Forschungsprojektes der Forschungsstelle Archäometrie Heidelberg durchgeführt, deren Ergebnisse bisher nur in der Dissertation KARELIN (1997) vorgestellt wurden. Sie beruhen maßgeblich auf einer methodischen Weiterentwicklung der Quarz-Thermolumineszenz-Datierung, sowie - auf dem Stand damaliger Kenntnisse - der Kalifeldspat- und Feinkorn-Thermolumineszenz-Datierung. Diese Daten werden vor allem dazu herangezogen, das Becken NN1 einem unbekannten älteren Interglazial zuzuordnen (zuletzt MANIA et al. 2010). Die Ergebnisse dieser Dissertation sind bisher nicht durch die Publikation in einschlägigen Fachzeitschriften der breiten Öffentlichkeit vorgestellt worden. Wesentlich ist, dass dadurch die Methodik der Datenerhebung, insbesondere einer sich auf experimentelle Weiter- 
entwicklungen stützenden, nicht ausreichend in Fachkreisen diskutiert ist. Diese Diskussion soll und kann auch hier nicht tiefgreifend geführt werden. Es finden sich jedoch ausreichend Gründe in der öffentlich zugänglichen Promotionsschrift (KARELIN 1997), dass eine Überbewertung der Genauigkeit der Daten durch die Zitierenden erfolgt, wobei Schwächen hinsichtlich einer transparenten Datenerhebung sowie Darstellung und Diskussion der Ergebnisse im angewandten geochronometrischen Teil der Arbeit auch zum Tragen kommen. Im Zusammenhang mit der Altersdiskussion zu NN1 soll deshalb auf die Datierungsergebnisse der Arbeit (KARELIN 1997) kurz eingegangen werden. Für die Zuordnung der Proben werden hier und im Weiteren die lithologischen Einheiten nach MANIA (2004) (siehe auch MANIA et al. 2008, 2010 und Abb. 14) benutzt. Wenn nicht anders vermerkt, werden die in der Lumineszenzdatierung üblichen 1- $\sigma$ Fehler (68\% Wahrscheinlichkeit) der TL-Alter aus KARELIN (1997) zitiert.

1. In der o. g. Literatur wird ein Datum von $184 \pm 17$ ka für das Alter der Warmzeit von NN1 zitiert, welches einem sogenannten „TL-Kontextalter“ (KARELIN 1997) entspricht. Der Wert dieser Angabe aus 4 Einzelaltern von 2 Proben (N 2, $\mathrm{N} 3^{5}$ ) aus Schichtkomplex 6 kann zunächst nicht eingeschätzt werden, da eine Berechnungsgrundlage eines „kombinierten, fehlergewichteten mittleren Alters“ nicht dargestellt ist und es dafür keine allgemein gültige Definition gibt. Es handelt sich aber offenbar einfach um ein fehlergewichtetes Mittel der Werte, wie leicht nachzurechnen ist, und nicht wie in der Lumineszenzdatierung üblich, um einen Standardfehler eines Probenkontext nach Aitken (1985). Diese Berechnung kann hier aufgrund fehlender Informationen nicht nachvollzogen werden, würde aber wegen der gesonderten Einbeziehung von zufälligen und systematischen Fehlern einen höheren Fehler erwarten lassen. Abgesehen davon fehlen aber Kontrollrechnungen, ob eine Mittelwertbildung überhaupt durchgeführt werden sollte. Führt man diese nach GEYH (2008) durch, kommt man diesbezüglich $\mathrm{zu}$ einem negativen Ergebnis. Für Schichtkomplex 6 sind daher eher die einzelnen Alter (N 2: 182 \pm 44; N 3: $195 \pm 35,184 \pm 27,174 \pm 34 \mathrm{ka}$ ) und deren Fehler als repräsentativ anzusehen. Diese überstreichen mit ihren Fehlern den Zeitraum von $230 \mathrm{ka}$ bis $138 \mathrm{ka}$, bei $95 \%$ Wahrscheinlichkeit von 265 bis 98 ka. Eine Einstufung des Interglazials in MIS 5e ist somit durchaus möglich, gleichwohl jedoch auch in MIS 7, wogegen jedoch die Unterlagerung des Beckens durch die Erste Saale-Grundmoräne spricht. Die geringe Bedeutung der Angaben zu „mittleren Altern“ durch den Autor kann anhand der Probe N 4 (Komplex 12) noch deutlicher gemacht werden. Hier wird aus Werten von $178 \pm 22 \mathrm{ka}$ und $120 \pm 17 \mathrm{ka}$, die also einen Bereich von 200 ka bis 103 ka überdecken, ohne dass sich die Werte im Fehlerbereich überlappen, unzulässig ein Wert von 142 \pm 14 ka berechnet, welcher dann als Nachweis für warthezeitliche Periglazialsedimente dienen soll, die gleichwohl auch frühweichselzeitlich sein können. Dazu ist auch anzumerken, dass laut KARELIN (1997: 73 f.) Dosisbestimmungen an zwei Chargen der gleichen Probe durchgeführt wurden, wobei keine hinreichende Begründung für die Differenz

5 Um Verwechslungen mit den Beckenbezeichnungen NN1, NN2 und NN3 zu vermeiden, wurden die Probennummern von Karelin (1997) geändert: N 1 statt NN 1, N 2 statt NN 2 usw. der Dosiswerte gefunden werden konnte. Der niedrigere Wert mit einem resultierenden TL-Alter von $120 \pm 17 \mathrm{ka}$ wäre unter Berücksichtigung der TL-Eigenschaften aber als zuverlässiger zu bewerten und damit eine Einstufung von Komplex 12 in MIS 6 nicht mehr zu belegen.

2. Alle Daten beruhen aufTL-Messungen. TL-Signale sind nur relativ schwer und nur bis zu einem unveränderlichen Restsignal durch Lichtexposition rückstellbar. Dadurch verbleiben in der Natur zum Zeitpunkt der Sedimentation leicht Restsignale, die zu Altersüberschätzungen besonders von TL-Altern führen können. Die Bemühungen und Ergebnisse von KARELIN (1997) insbesondere an Quarz-TL-Signalen günstigere Bedingungen für Sedimentdatierungen zu finden, sind hoch einzuschätzen. Die Ergebnisse reichen jedoch nicht für eine sichere Datierung, wie der Autor selbst nachweist. In einer Studie an Blindproben in dieser Arbeit, werden ohne Ausnahme Altersüberschätzungen mit der gleichen TLMethode an Quarz festgestellt, mit der auch die Proben N 1 und N 3 datiert wurden. Diese erreichen bis zu $60 \mathrm{ka}$ (!). Bei einem Doppelblindversuch an einer ca. 40-45 ka alten Probe (gestützt durch ${ }^{14} \mathrm{C}$ - und IRSL Alter) wurden $51 \pm 8 \mathrm{ka}$ und $107 \pm 20$ ka bestimmt, was zudem weitere Schlüsse (siehe bereits N 4) auf eine schlechte Reproduzierbarkeit der Daten zulässt. Leicht nachvollziehbar sind deshalb Altersüberschätzungen durch unzureichende Rückstellung des TL-Signals zum Zeitpunkt der Sedimentation. Das kann durch die neuen Altersbestimmungen untermauert werden (6.1.2).

3. Auf die Methodik der TL-Datierung an Feldspat (Kalifeldspat-Grobkorn bzw. polymineralische Feinkornfraktion, wegen der Dominanz der TL von Feldspat) wird in der Arbeit nicht ausreichend eingegangen. Deshalb ist eine Bewertung kaum möglich. Einen sichtbaren methodischen Mangel der damaligen Zeit stellt jedoch die Kalifeldspat-Altersberechnung von Probe N 3 (Schichtkomplex 6, $195 \pm 35$ ka) mit einem gemessenen Kaliumgehalt von 5,3 \% dar. Ein solches Messergebnis lässt auf eine nicht ausreichende Mineraltrennung und eine „Verdünnung“ der Kaliumbestimmung durch Verunreinigungen (vor allem Quarz) vermuten (DüTsCH \& KRBETSCHeK 1997). Bei Annahme eines durchschnittlichen Kaliumgehalts (11,5-13,5\%) gut separierter Kalifeldspäte, die trotz möglicher Verunreinigungen den Hauptteil des TL-Signales liefern (siehe z. B. HuntLEY \& BARIL 1998), ergibt sich ein Alter von etwa $153 \mathrm{ka}$ für diese Probe. Im Fehlerbereich überdeckt das Alter dann auch MIS 5e.

4. Die Problematik radioaktiver Ungleichgewichte (KRBETSCHEK et al. 1994), die häufig die Lumineszenzdatierung warmzeitlicher Sedimente ungünstig beeinflusst (DEgERING \& Krbetscher 2007a), konnte in der Arbeit KARELIN (1997) durch die angewandte Dosisleistungsanalytik nicht behandelt werden. Die neuen Ergebnisse weisen auf solche Verhältnisse in den beiden Sedimentbecken von Neumark-Nord hin (6.1.2). Damit sind noch unerkannte Altersfehler möglich, die die Quarz- und Feinkorn-Altersbestimmungen der Arbeit besonders beeinflussen (keine interne, konstante Dosisleistung aus ${ }^{40} \mathrm{~K},{ }^{87} \mathrm{Rb}$, wie bei Kalifeldspat). Konkret nachzuweisen ist diese Möglichkeit für Probe N 1 (Parallelprobe Neu 1, Tab. 6).

Zusammenfassend wird festgestellt, dass die TL-Altersbestimmungen der Arbeit von KARELIN (1997) an Sedimenten des Beckens NN1 von Neumark-Nord aus methodischer Sicht nicht dazu geeignet sind, eine Diskussion zu einem In- 
terglazial mit einem Alter um 180 ka zu führen. Die Daten an den Interglazialsedimenten und den Periglazialsedimenten im Hangenden lassen, ebenso wie sie wesentlich älter sein könnten, auch eine Einstufung in das Eem-Interglazial bzw. das Weichsel-Frühglazial zu. Angesichts der nicht ausgereiften Methodik der Quarz-TL-Datierung ist auch ein einzelnes Alter spätdrenthezeitlicher Sande (Probe N 1: $238 \pm 30 \mathrm{ka}$; Schichtkomplex 1) nicht von wesentlicher Bedeutung, besonders auch wegen möglicher unerkannter Dosisleistungsfehler.

\subsubsection{Neue Datierungen}

In den vergangenen ca. 15 Jahren wurden in den Sedimentbecken von Neumark-Nord mehrfach Beprobungen für Lumineszenzdatierungen mittels optischer Verfahren (IRSL, OSL) und später auch Radiofluoreszenz (IR-RF) durchgeführt. Die Probe Neu 1 (Tab. 6) ist eine Parallelprobe zu Probe N 1 von Karelin (1997). Weiterhin wurde eine Teilprobe der Probe N 3 von Karelin (1997, Tab. 6) untersucht. Erst im Jahre 2003 waren Teile von Becken NN1 (Basisschichten) begrenzt wieder zugänglich, zusammen mit denen in Becken NN3 abgelagerten prädrenthezeitlichen Sedimenten. Das war der Beginn einer stärkeren Einbindung der Sedimentsequenzen von Neumark-Nord in die Datierungsarbeiten der Forschungsstelle Geochronologie Quartär der Sächsischen Akademie der Wissenschaften am Institut für Angewandte Physik der TU Freiberg. Zwischen 2003 und 2007 wurden auch 24 Proben von Sedimenten des Beckens NN2 und dessen Hangendfolge entnommen. Bisher konnten nur Teile dieses umfangreichen Probenmaterials datiert werden. Hier werden im Wesentlichen nur die abgeschlossenen Datierungen am Becken NN1 vorgestellt und diskutiert, jedoch liegen auch für Becken NN2 Daten vor, die einer stratigraphischen Zuordnung dienen können.

Von den Sedimenten aus dem Becken NN1 liegen 4 Datierungsergebnisse vor (Tab. 6, Abb. 14). Zwei davon (Neu 1 und N 3), Parallel- bzw. Teilproben aus Karelin (1997), wurden in der zweiten Hälfte der 1990er Jahre mittels Infrarot-Optisch-Stimulierter Lumineszenz (IRSL) an Kalifeldspat und der MAA-Technik (multiple-aliquot additive dose; Wintle 1997) datiert. Die Diskussion über Fading von TL-, IRSL- oder OSL-Signalen, welche zu Altersunterschätzungen führen kann, ist nicht abgeschlossen (siehe z. B. ZöLLER 2010). Größere (über den angegebenen Fehlerbereich hinaus) Unterbestimmungen können hier aber mit hoher Wahrscheinlichkeit ausgeschlossen werden. Mit der gleichen Technik wurden zahlreiche, durch unabhängige Methoden kontrollierte Sedimentsequenzen bis in den Zeitbereich um 150 ka erfolgreich datiert (KRBETSCHEK \& Stolz 1997, KRBetschek et al. 1998, Degering \& KrbetscheK 2007a, b). Das IRSL-Signal wird in der Natur optisch relativ schnell gelöscht. Das verdeutlicht auch die geringe Streuung der IRSL-Messwerte beider Proben und ein sehr gutes Dosis-Plateau der Probe N 3 über den gesamten Bereich der Ausleuchtkurve („shine-down“).

Neuere Datierungsergebnisse liegen durch die Anwendung der Infrarot-Radiofluoreszenz (IR-RF) an 2 im Jahre 2003 gewonnenen Proben aus zwei Horizonten vor (Tab. 6: Pr. NMN 3 und NMN 4). Auch diese Methode basiert auf der Verwendung von Kalifeldspat, jedoch ist für das IR-RF-
Signal eine Signalstabilität nachgewiesen (z. B. ERfurt ひ Krbetschek 2003, Degering \& Krbetschek 2007a). Das IR-RF-Signal wird durch Lichtexposition etwas langsamer zurückgestellt als die IRSL. Probleme von Altersüberschätzungen können aber durch die single-aliquot-Technik (IRSAR-Protokoll) erfolgreich ausgeschlossen werden, die bei allen IR-RF-Datierungen von Neumark-Nord Anwendung fand. Unvollständige optische Rückstellung von Kornanteilen des Sediments wurden vor allem bei NMN 4 festgestellt. Da TL-Signale wesentlich höhere Lichtintensität bzw. Belichtungsdauer für ihre Rückstellung benötigen, können diese Ergebnisse auch Altersüberschätzungen der älteren TL-Daten (KARELIN 1997) als wahrscheinlich begründen. An zwei Proben (Tab. 6: Neu 1 und NMN 3) wurden geringe radioaktive Ungleichgewichte in der ${ }^{238} \mathrm{U}$ Reihe festgestellt. Von einer detaillierten Behandlung (Modellrechnung) konnte wegen der geringfügigen Auswirkung auf das Altersergebnis (angegebener Fehler; hohe interne Dosisleistung Kalifeldspat) abgesehen werden. An der QuarzFraktion der Proben NMN 3 und NMN 4 wurden auch OSLMessungen (SAR) zur Paläodosisbestimmung durchgeführt. Das OSL-Signal ist jedoch in Sättigung. Sogenannte „dose-recovery“-Tests im Bereich von 200-250 Gy zeigten starke Unterbestimmungen. Diese Methode erwies sich damit als ungeeignet, die Sedimente zu datieren.

Die Ergebnisse der Lumineszenz-Altersbestimmungen sind in Abb. 14 zusammengefasst. Sie scharen sich im unmittelbaren Liegenden des Interglazials (Schichtkomplexe 1 und 2) von NN1 bei ca. 140 ka. Die Daten der Proben NMN 3 und NMN 4 können als maximale Alter der limnischen Sedimentation angesehen werden. Im Fehlerbereich könnte diese frühestens vor ca. $150 \mathrm{ka}$ begonnen haben. Aus den Interglazialsedimenten (Schichtkomplex 6) liegt eine Datierung mit $132 \pm 12$ ka vor (Tab. 6: N 3). Auf der Basis der Zentralwerte und der zum Jüngeren reichenden Fehler ist von einer eemzeitlichen Beckensedimentation auszugehen.

Die Lumineszenzdatierungen an Sedimenten des Beckens NN2 sind trotz zahlreicher Messungen noch nicht abgeschlossen. Dies betrifft vor allem die Proben NMN 17 bis NMN 22 aus dem Hauptprofil A, so dass sich die Vorstellung der vorläufigen Ergebnisse vorrangig auf das Profil B1 bezieht (Abb. 4, Tab. 6).

Datierungen limnischer Sedimente mittels der QuarzOSL-SAR-Technik waren in NN2 weitgehend erfolglos. Wie in Becken NN1 ist das Signal wegen einer relativ hohen Dosisleistung in Sättigung (Pr. NMN 9 und NMN 12). Bei Probe NMN 12 aus dem Niveau des unteren Bereichs von Profil B2 (wenige Meter lateral versetzt vom Profil entnommen) kann gerade noch ein Mindestalter abgeschätzt werden. Mit $88 \pm 8$ ka (Pr. NMN 10) war jedoch ein sicherer Wert für den frühweichselzeitlichen archäologischen Fundhorizont NN2/0 im Profil B1 (Abb. 6) mit dieser Methode bestimmbar, da die Aktivität dieser sandigen Schicht relativ gering ist.

Bei allen Paläodosisbestimmungen, die durchgängig auf der Anwendung von Einzelpräparat-Techniken beruhen, ist eine sehr inhomogene, oft unzureichende Nullstellung der Signale zu beobachten. Diese ist wahrscheinlich auf die räumlich kleine Ausdehnung des Beckens zurückzuführen und die damit verbundene Möglichkeit des erhöhten Sedimenteintrags bei kurzen Transportdistanzen (z. B. aus der Uferzone). Das ist besonders bei IR-RF-Messungen zu be- 


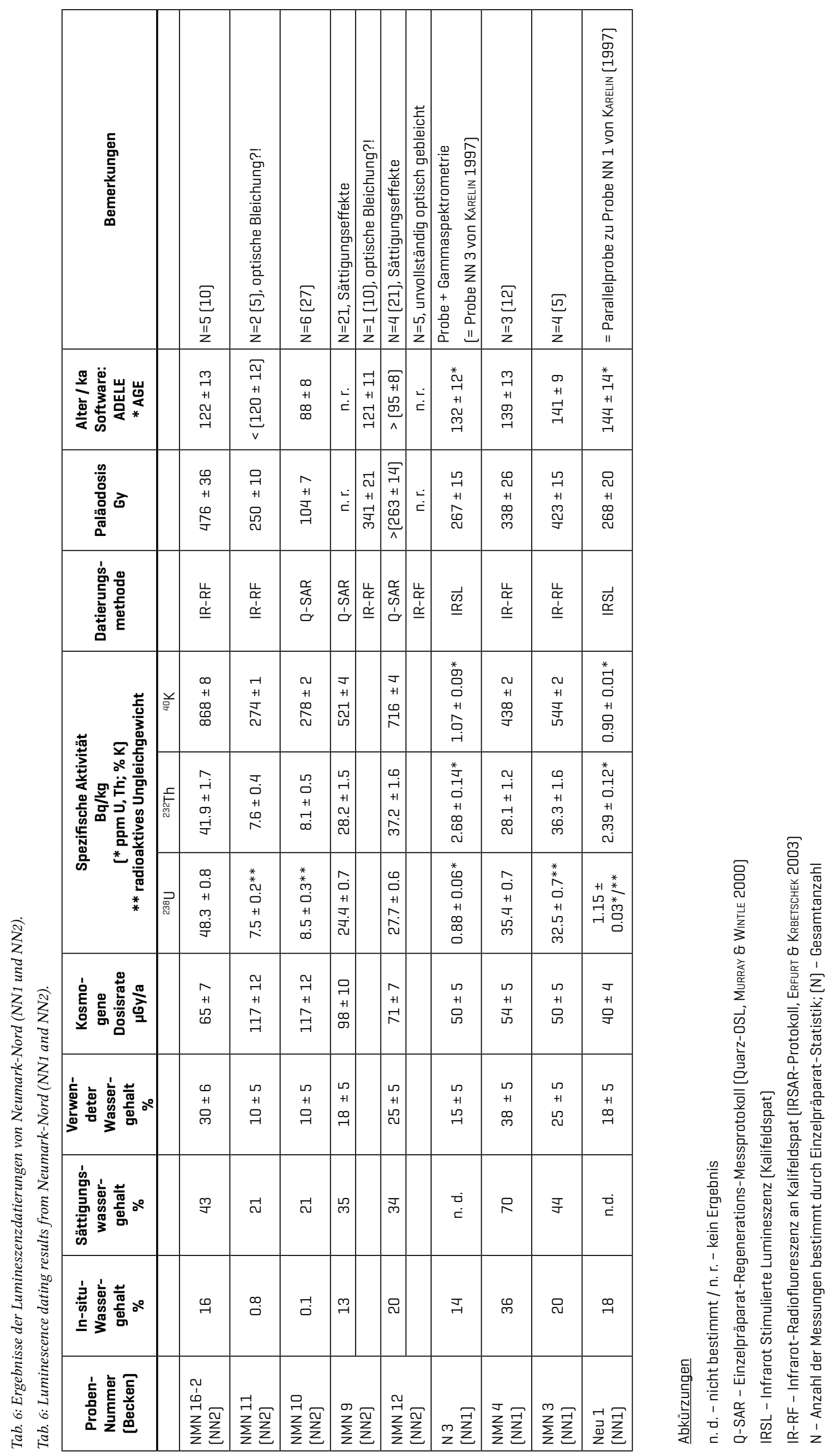


obachten, die viele überhöhte, teils nicht mehr bestimmbare Dosiswerte liefern (NMN 9 und NMN 12). Teils liegt noch keine ausreichend hohe Zahl von Messungen vor, um das Problem der optischen Rückstellung genauer zu fassen (NMN 11, aus gleicher Schicht wie NMN 10, Abb. 4). Ein etwas besser gesichertes Alter von $121 \pm 11$ ka (NMN 9) ergibt sich für den oberen Abschnitt der Beckensedimentation. Jedoch bedarf auch hier die statistisch saubere Fassung gut optisch gebleichter Kornanteile weiterer Messungen. Ebenfalls erbrachten die IR-RF-Datierungsmessungen der Probe NMN 16-2 (Übergang Saale-Spätglazial/Eem-Interglazial) bereits ein recht gut gesichertes Alter von $122 \pm 13$ ka.

Die bisher gewonnenen Daten zum Alter der Sedimente des Beckens NN2 machen eine Korrelation mit dem Eem bzw. MIS 5e wahrscheinlich. Das wird auch durch Ergebnisse noch laufender Untersuchungen mittels Kalifeldspat-IRSLDatierung an den Universitäten Bern und Delft bestätigt (freundl. mündl. Mitt. F. Preusser bzw. J. Wallinga). Diese und weitere Ergebnisse der drei beteiligten Laboratorien werden nach deren Abschluss Gegenstand einer gemeinsamen Publikation sein. Diese soll auch besondere Einflüsse der Sedimentation für die Methodik der Lumineszenzdatierung tiefgründiger erschließen.

\subsection{Aminosäuren-Geochronologie im Becken NN2}

\section{[B. Machalett \& E. A. Oches]}

D/L-Verhältnisse von Glutaminsäure (D/L-Glu) gemessen an fossilen Gehäusen von Gyraulus laevis ergaben im Profilabschnitt 95-101 m NN relativ einheitliche Werte, die sich in einem Spektrum von 0,162 (Pr. AAR 3) bis 0,183 (Pr. AAR 23) bewegen. Ausnahmen bilden die Proben AAR 4, AAR 21 und AAR 22, die niedrigere D/L-Werte aufweisen (Abb. 15, Tab. 7). Für alle drei Proben liegen die D/L-Werte 20-30 \% unter dem Mittelwert aller D/L-Glu-Werte von Gyraulus laevis im beprobten Profilabschnitt und liegen damit außerhalb der $1 \sigma$ Standardabweichung aller Gyraulus laevis-Proben. Die qualitative Betrachtung der Messergebnisse der AAR-Analysen ergibt keine Hinweise für die nied- rigeren D/L-Verhältnisse der drei Proben. Insbesondere die abweichenden Werte der Probe AAR 4 sind damit schwer erklärbar, da sich Störfaktoren wie eine zusätzliche Temperaturhistorie gewöhnlich in höheren D/L-Werten äußern würden. Allerdings können bestimmte Bedingungen im Sedimentationsmilieu zu einer Pufferung des initialen Razemisierungsvorgangs geführt haben, was aber im Rahmen der vorliegenden Untersuchung nicht abschließend geklärt werden kann. Fehler bei der taxonomischen Auswahl können weitgehend ausgeschlossen werden, da es für aminostratigraphische Untersuchungen ausreicht, Probenabfolgen einer einzelnen Gattung zu untersuchen, während hier das Probenspektrum auf eine Art beschränkt ist.

Im Gegensatz zu Probe AAR 4, weisen die Werte der Proben AAR 21 und AAR 22 auf einen klaren stratigraphischen Zusammenhang hin, und deuten durch die niedrigeren D/L-Verhältnisse das Ende des zusammenhängenden Sedimentationszyklus an. Durch die konsistenten Werte der Proben AAR 21 und AAR 22 müssen die D/L-Glutaminsäure-Werte der stratigraphisch jüngsten Probe AAR 23 kritisch betrachtet werden. Die zunehmende klastische Sedimentation und die oxidativen Bedingungen in diesem stratigraphischen Abschnitt haben mit großer Wahrscheinlichkeit zu störenden Einflüssen und einer zusätzlichen Temperaturhistorie geführt, so dass die D/L-Glu-Werte der Probe AAR 23, die eigentlich im Bereich der AAR 21 und AAR $22 \mathrm{zu}$ erwarten wären, deutlich höher ausfallen.

Bis auf die Probe AAR 4 deutet die Konformität der D/LGlutaminsäure-Werte in dem beprobten Profilabschnitt insgesamt auf eine quasikontinuierliche sedimentäre Abfolge hin, die in ihrer Gesamtheit einem Interglazial- bzw. Glazialabschnitt zuordenbar ist. Da sich selbst kurze, zwischengeschaltete Warm- oder Kaltphasen (Interstadial/Stadial) durch einen signifikanten, wenn auch geringen Anstieg der D/L-Verhältnisse äußern würden (vgl. MACHALETT et al. 2008), deuten die plateauartigen $\mathrm{D} / \mathrm{L}$-Verhältnisse von Gyraulus laevis (Probe AAR 3 bis AAR 20) zudem auf einen Ablagerungszeitraum innerhalb eines MIS-Substadiums hin.

Tab. 7: Ergebnisse des Aminosäuren-Geochronology. Angegeben sind D/L-Glutaminsäure-Werte (Totalhydrolysat) im Mittel $\pm 1 \sigma$ Standardabweichung gemessen an Gyraulus laevis-Gehäusen von Neumark Nord (NN2), vgl. Abb. 15.

Tab. 7: Results of Amino acid geochronology. Summary total hydrolysate amino acid racemization data for D/L-Glutamic Acid measured in Gyraulus laevis shells from Neumark Nord (NN2), see fig. 15.

\begin{tabular}{|c|c|c|c|}
\hline $\begin{array}{l}\text { Proben- } \\
\text { nummer }\end{array}$ & $\begin{array}{c}\text { FAL } \\
\text { Labornummer }\end{array}$ & $\begin{array}{c}\text { D/L - Glutaminsäure } \\
\text { Mittel } \pm 1 \sigma\end{array}$ & $\begin{array}{l}\text { Anzahl der } \\
\text { Teilproben }\end{array}$ \\
\hline AAR 23 & 2154 & $0.183 \pm 0.021$ & 3 \\
\hline AAR 22 & 2153 & $0.128 \pm 0.015$ & 2 \\
\hline AAR 21 & 2148 & $0.121 \pm 0.024$ & 4 \\
\hline AAR २० & 2147 & $0.175 \pm 0.036$ & 4 \\
\hline AAR 19/1 & 2146 & $0.170 \pm 0.021$ & 5 \\
\hline AAR 19/2 & 2152 & $0.183 \pm 0.017$ & 5 \\
\hline AAR 6 & 2145 & $0.177 \pm 0.007$ & 3 \\
\hline AAR 5 & 2151 & $0.171 \pm 0.034$ & 5 \\
\hline AAR 4 & 2150 & $0.118 \pm 0.011$ & 4 \\
\hline AAR 3 & 2149 & $0.162 \pm 0.028$ & 4 \\
\hline $\mathrm{NH} 1$ & 2143 & $0.171 \pm 0.017$ & 5 \\
\hline $\mathrm{NH2}$ & 2144 & $0.175 \pm 0.008$ & 4 \\
\hline
\end{tabular}




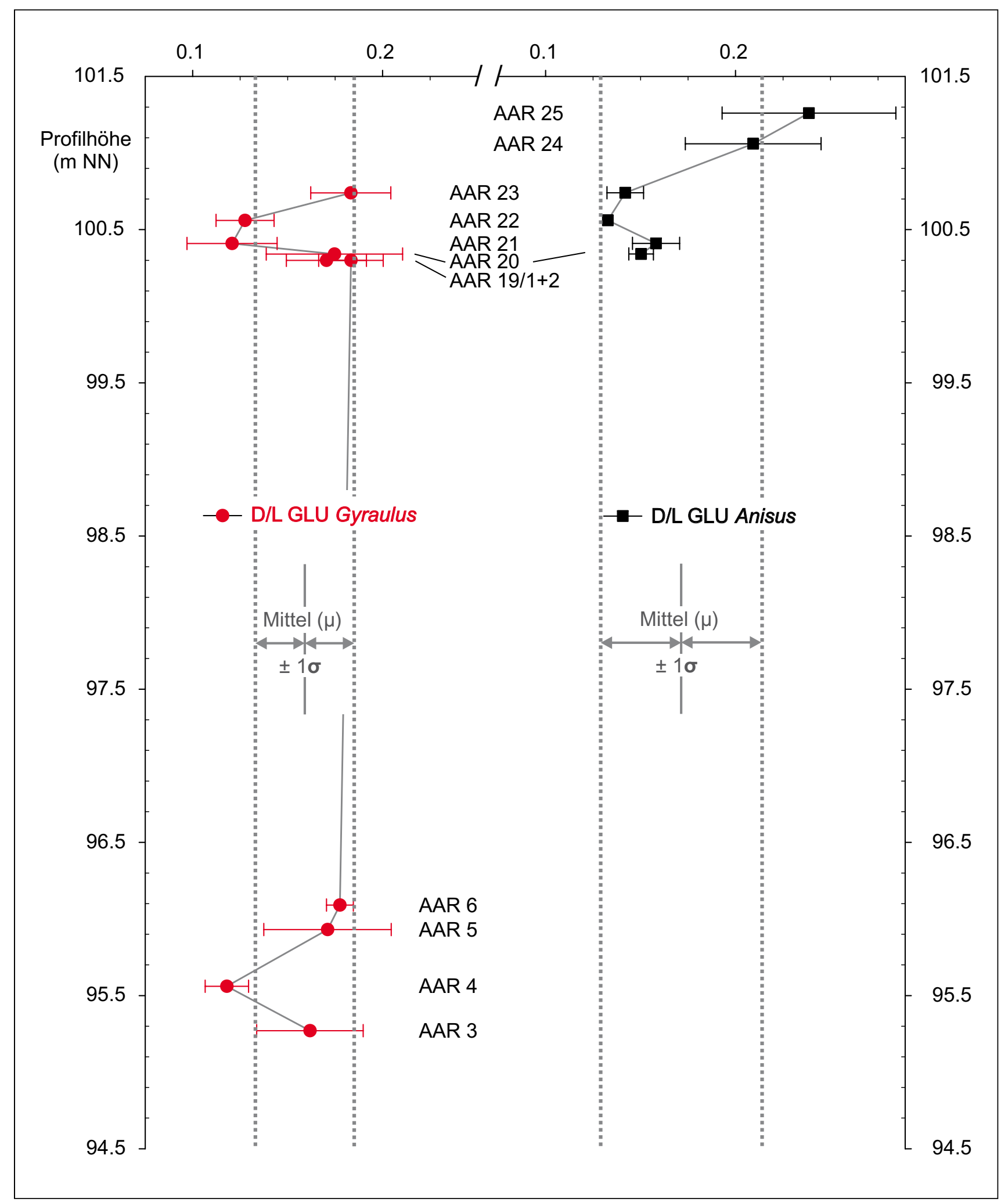

Abb. 15: D/L-GLU(Glutaminsäure)-Verhältnisse von Gyraulus laevis und Anisus leucostoma. Für eine Übersicht der gemessenen D/L-GLU-Werte siehe Tab. 7 und Tab. 8.

Fig. 15: D/L Glutamic acid data are mean and one standard deviation total acid hydrolysate values measured in Gyraulus laevis and Anisus leucostoma shells. Summary $D / L$ data are shown in table 7 and table 8.

Diese Ergebnisse werden durch Resultate von D/L-Glutaminsäure-Verhältnissen, gemessen an Anisus leucostomaGehäusen, aus dem Profilabschnitt 100,0-101,5 m NN gestützt (Abb. 15, Tab. 8). D/L-Werte der Proben AAR 20-23 bewegen sich in einem Bereich von 0,133 bis 0,158 und kennzeichnen damit, unter Berücksichtigung taxonomi- scher Einflüsse, einen ähnlichen Ablagerungszeitraum wie D/L-Glu von Gyraulus laevis, wobei auch hier die stratigraphisch jüngere Probe (AAR 22) leicht niedrigere D/L-Verhältnisse aufweist, und die D/L-Werte der Probe AAR 23 durch störende Einflüsse des Paläoumweltmilieus überprägt sind. 
Tab. 8: Ergebnisse des Aminosäuren-Geochronology. Angegeben sind D/L-Glutaminsäure-Werte (Totalhydrolysat) im Mittel $\pm 1 \sigma$ Standardabweichung gemessen an Anisus leucostoma-Gehäusen von Neumark Nord (NN2), vgl. Abb. 15.

Tab. 8: Results of Amino acid geochronology. Summary total hydrolysate amino acid racemization data for D/L-Glutamic Acid measured in Anisus leucostoma shells from Neumark Nord (NN2), see fig. 15.

\begin{tabular}{|c|c|c|c|}
\hline $\begin{array}{c}\text { Proben- } \\
\text { nummer }\end{array}$ & $\begin{array}{c}\text { FAL } \\
\text { Labornummer }\end{array}$ & $\begin{array}{c}\text { D/L - Glutaminsäure } \\
\text { Mittel } \pm \text { 10 }\end{array}$ & $\begin{array}{c}\text { Anzahl der } \\
\text { Teilproben }\end{array}$ \\
\hline AAR 25 & 2443 & $0.239 \pm 0.046$ & 5 \\
\hline AAR 24 & 2442 & $0.209 \pm 0.036$ & 3 \\
\hline AAR 23 & 2441 & $0.142 \pm 0.010$ & 4 \\
\hline AAR 22 & 2440 & $0.133 \pm 0.002$ & 5 \\
\hline AAR 21 & 2445 & $0.158 \pm 0.012$ & 4 \\
\hline AAR 20 & 2444 & $0.150 \pm 0.006$ & 4 \\
\hline
\end{tabular}

Abweichend dazu zeigen sich die Ergebnisse, die an den beiden obersten Proben von Anisus leucostoma gemessen wurden. D/L-Glu-Werte der Proben AAR $24(0,209 \pm 0,036)$ und AAR $25(0,239 \pm 0,046)$ offenbaren einen deutlichen Anstieg von ca. $50 \%$ gegenüber den stratigraphisch älteren Proben AAR 20-23. Damit verhalten sich die Werte dieser beiden Proben genau gegensätzlich als für stratigraphisch jüngere Proben zu erwarten wäre, und deuten, auch aufgrund der relativ hohen Standardabweichung der Proben AAR 24 und AAR 25, auf eine Inkorporation von stratigraphisch älterem Material (prä-eemzeitlich) hin. Aus sedimentologischer Sicht ist im obersten Profilabschnitt durchaus mit lateralen Umlagerungsprozessen zu rechnen (Verzahnungsbereich von Seeablagerungen und Fließerde, siehe 4.3.1), nach palynologischen und malakologischen Befunden allerdings ohne nachweisbare Beteiligung von prä-eemzeitlichem Material. Außerdem kommt Anisus leucostoma in den untersten (saalezeitlichen) Profilabschnitten nicht vor (Abb. 12). Eine endgültige Interpretation der D/L-Glu-Werte der Proben AAR 24 und AAR 25 muss daher weiteren Untersuchungen vorbehalten bleiben.

Da das besondere Potential der Aminosäuren-Stratigraphie vor allem in der Möglichkeit besteht, durch den Vergleich mit stratigraphisch gesicherten (Typus-)Profilen regionalstratigraphische Korrelationen und relative Altersmodelle zu etablieren, wurden für die vorliegende Untersuchung Anisus leucostoma-Proben aus den eemzeitlichen Travertinaufschlüssen Weimar, Belvedereallee (Villa Cosima) und Burgtonna/Gräfentonna in Thüringen zur Gegenüberstellung herangezogen (vgl. KAHLKE 2002 und MANIA et al. 2003). Diese Proben sind Gegenstand einer laufenden Kooperation und umfassenden aminostratigraphischen Untersuchung mitteldeutscher Travertine der Autoren Machalett, Meng und Oches, initiiert durch Prof. William D. McCoy (Geoscience Department, University of Massachusetts, Amherst) und in Zusammenarbeit mit Dr. Lutz Maul (Forschungsinstitut Senckenberg, Forschungsstation für Quartärpaläontologie, Weimar). Sie werden in späteren Publikationen eingehender betrachtet.

D/L-Glutaminsäure-Verhältnisse, gemessen an Anisus leucostoma-Proben, aus den als eemzeitlich gesicherten Travertinsanden des Aufschlusses Weimar, Belvedereallee (Villa Cosima) ergaben Werte in einem Spektrum von 0,144 $\pm 0,011$ (Probe FAL 2427). D/L-Glu-Werte an der Probe FAL 2417 aus dem Aufschluss Burgtonna/Gräfentonna liegen in einem ähnlich engen Bereich bei 0,153 $\pm 0,017$.
Eine Korrelation mit den Anisus leucostoma-Proben AAR 20-23 in NN2 verweist auf eine Sedimentation des Beckens, die zeitgleich mit der Travertinbildung in den thüringischen Profilen stattfand und belegt damit ein Sedimentationsalter während des MIS 5. Diese Aussage wird vor allem durch die konsistenten D/L-Glu-Werte von Gyraulus laevis (Probe AAR 3 bis AAR 20) gestützt, die für eine überwiegend zusammenhängende Sedimentationsphase innerhalb eines Interglazialzykluses sprechen, so dass aufgrund der aminostratigraphischen Ergebnisse der Kernbereich des Hauptprofils A (ca. 95-101 m NN) dem MIS 5 zuzuordnen ist. Dies wird auch durch die D/L-Glu-Werte von Gyraulus laevisProben aus dem archäologischen Hauptfundhorizont NN2/2 vom Beckenrand (vgl. 5.3) gestützt, die sich mit Werten von $0,171 \pm 0,017(\mathrm{NH} 1)$ und $0,175 \pm 0,008(\mathrm{NH} 2)$ eindeutig mit den D/L-Glu-Verhältnissen im Hauptprofil A korrelieren lassen.

\section{Fazit}

Mit dem Hauptprofil A liegt das am vollständigsten untersuchte Profil des Zentralteils des Paläoseebeckens NN2 vor. Einschließlich der Rammkernsondierung reicht es von 90,4 bis $101,4 \mathrm{~m} \mathrm{NN}$. Die interdisziplinären Untersuchungen an diesem und den benachbarten Profilen zeigen übereinstimmend, dass die limnische Sedimentation im Becken NN2 vom Ende des Saale-Komplexes über die Eem-Warmzeit bis in die Weichsel-Kaltzeit erfolgte. Das Profil lässt Seespiegelschwankungen mit einer generellen Tendenz der Verflachung und Verlandung sowie wechselnde Sedimentationsraten erkennen. Durch die palynologischen Untersuchungen sind außerdem mit Erosion und Sedimentumlagerungen verbundene Hiaten festgestellt worden.

Der archäologische Hauptfundhorizont NN2/2, der von LAURAT \& BRÜHL (2006), LAURAT et al. (2006) und MANIA et al. $(2008,2010)$ einer von der Eem-Warmzeit durch eine Kaltphase abgetrennten prä-eemzeitlichen Warmzeit zugeordnet wurde, ist in der Eem-Warmzeit akkumuliert worden.

Vor allem die palynostratigraphische, aber auch die malakologische Koinzidenz der Becken NN1 und NN2 belegt die Gleichaltrigkeit der Ablagerungen. Gegenteilige palynologische Argumente sind erneut entkräftet worden. Somit existiert in Neumark-Nord zwischen der Saale-Grundmoräne der Zeitz-Phase und der Weichsel-Kaltzeit nur eine Warmzeit, das Eem. 
Die Ergebnisse neuerer Lumineszenzdatierungen weisen ebenfalls auf eine Zeitgleichheit der Beckenfüllungen und eine Zuordnung zum Eem-Interglazial (MIS 5e, ca. 127-115 ka) hin. Ältere Thermolumineszenz-Daten von NN1 stehen durch ihre geringe Genauigkeit dazu nicht zwingend im Widerspruch. Wegen des damaligen experimentellen Standes der angewandten Methodik, die vor allem Möglichkeiten der Altersüberschätzung aufweist, sind sie nicht dazu geeignet, das Becken von Neumark-Nord 1 in ein älteres Interglazial einzustufen.

Das Alter des Saale-Hochglazials (Drenthe-Stadium und Warthe-Stadium) ist in Mittel- und Ostdeutschland mit ca. 150-130 ka bestimmt worden (KRBETSCHEK \& EISSMANN 2008, KRBEtscheK et al. 2008). Dieser Zeitabschnitt ist somit in das MIS $6 \mathrm{zu}$ stellen, was in Daten aus drenthezeitlichen Ablagerungen der Niederlande Bestätigung findet (Busschers et al. 2008). Pollenanalytisch dem Eem zugewiesene Sedimente, sowohl auf drenthe- als auch auf warthestadialen Sedimenten, konnten stets durch geochronometrische Datierungen dem MIS 5e zugeordnet werden (z. B. KRBETSCHEK \& STOLZ 1994, 1997, DEgERING \& KRBETSCHEK 2007 a, b). Diese Zeitmarken setzen Grenzen, die weitere Klimaschwankungen vergleichbaren Ausmaßes aus gegenwärtiger paläoklimatisch-quartärgeologischer Sicht nicht zulassen. Dieser Umstand untermauert die durch neue Datierungen fixierte Geochronologie der Sedimentbecken von NeumarkNord (NN1 und NN2) und damit deren eemzeitliche Genese.

Die aminostratigraphischen Untersuchungen belegen zum einen die quasikontinuierliche Sedimentation des zentralen Abschnitts des Paläoseebeckens NN2, zum anderen erlaubt die Aminosäuren-Stratigraphie eine direkte Korrelation des Beckens NN2 mit anderen eemzeitlich eingestuften Sedimentsequenzen Mitteldeutschlands und verweist damit auf eine sedimentäre Genese von NN2 während des MIS 5.

Die Einstufung des Beckens NN1 in die Eem-Warmzeit wird auch durch vergleichende isotopen-geochemische Untersuchungen an mehreren Profilen in Mitteldeutschland gestützt (BoEtTGer et al. 2005, 2009). Die auf einer angeblichen mündlichen Mitteilung von T. BoetTGer beruhende Aussage von MANiA et al. (2008), dass die Becken NN1 und NN2 aufgrund von isotopen-geochemischen Analysenergebnisse nicht gleichzeitig entstanden sein könnten, ist unzutreffend (freundl. mündl. Mitt. T. BoETTGER, Halle).

Schließlich ist $\mathrm{zu}$ konstatieren, dass die Stratigraphie von Neumark-Nord im Einklang mit der Chronostratigraphie des Pleistozäns steht. Vergleichbar mit dem Profil der Bohrung Amsterdam-Terminal, das als GSSP für die Basis des Oberpleistozäns vorgeschlagen wurde (LITT \& GIBBARD 2008), lagern hier Sedimente der Eem-Warmzeit direkt auf Sedimenten des Saale-Komplexes.

Die von MANiA et al. $(2008,2010)$ dokumentierten Lagerungsbeziehungen, nach denen die Beckenfolge NN2 über der von NN1 liegen soll, kann nicht bestätigt werden. Weitere von MANIA wiederholt angeführte Argumente für ein intrasaalezeitliches Alter von NN1 haben keine hinreichende stratigraphische Relevanz (vgl. LitT 1994a, b). Das betrifft die Ausbildung der periglaziären Deckschichten ebenso wie die Zusammensetzung der Säugetierfauna bzw. ihrer z. T. fraglichen phylogenetischen Merkmale, die unter Missachtung der geologischen Verhältnisse am Fundort stratigraphisch fehlinterpretiert wurden (u. a.
VAN DER MADE 2003, 2010). Insbesondere ist der Versuch von Mania et al. (2008) und HeINRICH (2010) abzulehnen, das mittelpleistozäne Alter von NN1 mit dem Nachweis der Zwergwaldmaus Apodemus maastrichtiensis belegen zu wollen, die nach ihrer Auffassung im Eem nicht gelebt hat (vgl. auch HeInRICH 2001). Apodemus maastrichtiensis ist jedoch auch aus dem Interglazialvorkommen von Grabschütz bekannt (BENECKE et al. 1990), dessen eemzeitliche Einstufung seit langem gesichert ist (u. a. LiTt 1990, 1994a). Somit stellt der Fund in Neumark-Nord nur einen zusätzlichen Beleg für die Existenz dieser Maus während der EemWarmzeit dar.

Die Makroflorenreste (MAI 1990) und die Ostrakoden (zuletzt FuHrmann 2006) sind hervorragende Bioindikatoren für die Paläoumweltbedingungen. Sie erlauben somit Rückschlüsse auf die spezifischen Standortverhältnisse, bilden jedoch keine geeigneten Kriterien für die Definition neuer klimatostratigraphischer Einheiten. In Neumark-Nord werden die klimatischen Besonderheiten des Mitteldeutschen Trockengebietes während der Eem-Warmzeit deutlich. Insofern bietet der Vergleich der Eem-Vorkommen von Neumark-Nord, Gröbern und Grabschütz ein Lehrbeispiel für die standortspezifische Variabilität benachbarter synchroner Warmzeitprofile (vgl. LiTT 1994a).

\section{Dank}

Die vorgelegten Untersuchungen wurden zum größten Teil an Schurfprofilen des Landesamtes für Denkmalpflege und Archäologie Sachsen-Anhalt (LDA) durchgeführt. Dafür danken wir dem Direktor des Amtes Herrn Prof. Dr. H. Meller, der unsere Aktivitäten stets wohlwollend begleitet und gefördert hat. Seit 2003 hat sich zwischen den archäologischen Bearbeitern des LDA und den Autoren dieses Beitrages eine fruchtbare Kooperation entwickelt. Dies begann zunächst mit wertvollen Kontakten mit dem langjährigen Leiter der wissenschaftlichen Bearbeitung der archäologischen und paläontologischen Fundstätte Neumark-Nord und Entdecker des Beckens NN2, Herrn Prof. Dr. D. Mania, dem wir auch für die Bereitstellung seiner Geländedokumentationen von 2003/2004 Dank schulden. Des Weiteren danken wir insbesondere den Kollegen E. Brühl und T. Laurat für ihre zahlreichen sachkundigen Erläuterungen zur Geologie, Paläontologie und Archäologie von Neumark Nord, für den kollegialen Datenaustausch und für viele konstruktive Diskussionen, an denen z. T. auch weitere Mitglieder des Grabungsteams, vor allem Herr N. Hesse, beteiligt waren. Wertvolle Hinweise und Anregungen erhielten wir außerdem von Prof. Dr. M. Altermann (Halle), Dr. T. Böttger (Halle), Prof. Dr. L. Eißmann (Leipzig), PD Dr. F. W. Junge (Leipzig), Prof. Dr. T. Litt (Bonn), Dr. R. Ruske (Halle), K. Sommerwerk (Halle), Dr. M. Thomae (Halle) u. a. Für die Bearbeitung paläontologischen Fundmaterials danken wir den Kollegen Dr. G. Böhme (Berlin), Dr. L. Maul (Weimar) und Dr. U. Bößneck (Erfurt). Unser Dank gilt auch den Herren Prof. Dr. C. Lempp und J. Buchantschenko (Halle) für die Durchführung der Rammkernsondierung im Rahmen eines studentischen Praktikums an der MLU und für Laboranalysen am Kernmaterial. Die Korngrößenzusammensetzungen, Kalk- und Humusgehalte der Proben aus den Schurfprofilen wurden von Frau C. Fleischer und Mitarbeiterinnen (Labor 
des LAGB Sachsen-Anhalt) bestimmt. Ihnen sei ebenso gedankt wie Frau L. Musch (Landeslabor Berlin-Brandenburg) für die Anfertigung der Pollenpräparate. Des Weiteren danken wir folgenden, zeitweise an den Geländearbeiten beteiligten Kolleginnen und Kollegen: A. Burmeier, K. Schuberth (LAGB Sachsen-Anhalt), N. Hermsdorf, Dr. N. Schlaak, A. Sonntag und Dr. H.-U. Thieke (LBGR Brandenburg).

\section{Literatur}

Ad-Hoc-Arbeitsgruppe Boden des Bund-Länder-Ausschusses BoDENFORSCHUNG (BLA-GEO) (2005): Bodenkundliche Kartieranleitung (KA5). - 438 S.; Hannover.

Ad-Hoc-Arbeitsgruppe Geologie des Bund-LÄnder-Ausschusses BoDENFORSCHUNG (BLA-GEO): Geologische Kartieranleitung der Staatlichen Geologischen Dienste, Teil Fachliche Grundlagen: http://www. geol-ka.de

Aitken, M. J. (1985): Thermoluminescence dating. - 359 S.; London (Academic Press).

Benecke, N., BöHme, G. \& HeInRICH, W.-D. (1990): Wirbeltierreste aus interglazialen Beckensedimenten von Gröbern (Kr. Gräfenhainichen) und Grabschütz (Kr. Delitzsch). - Altenburger naturwiss. Forsch., 5: 231281

Benkert, D., Fukarek, F. \& Korsch, H. (Hrsg.) (1996): Verbreitungsatlas der Farn- und Blütenpflanzen Ostdeutschlands. - 615 S.; Jena (Gustav Fischer).

Boettger, T., Junge, F. W., Knetsch, S., Krebetschek, M. \& Kremenetski, K. V. (2005): Limnic Sediment Sequence from Neumark-Nord in Central Germany: Environmental Changes and Stratigraphic Connection to Eemian Interglacial. - Posterhandout Projekt DEKLIM, $1 \mathrm{~S}$.

Boettger, T., Junge, F. W., Knetsch, S., Novenko, E. Y., Borisova, O. K., KREMENETSKi, K. V. ש VelichKo, A. A. (2007): Indications of short-term climate warming at the very end of the Eemian in terrestrial records of Central and Eastern Europe. - In: Sirocko, F., Claussen, M., Sánchez Goni, M. F. \& Litt, T. (eds.), The Climate of Past Interglacials, Developments in Quaternary Science, 7: 265-275; Elsevier (Amsterdam).

Boettger, T., Novenko, E. Y., Velichko, A. A., Borisova, O. K., KremeNETSKI, K. V., KNETSCH, S. \& JUNGE, F. W. (2009): Instability of climate and vegetation dynamics in Central and Eastern Europe during the final stage of the Last Interglacial and Early Glaciation. - Quaternary International, 207: 137-144.

BöнмE, G. (2010): Reste von Fischen aus der interglazialen Schichtenfolge von Neumark-Nord bei Merseburg. - Veröffentlichungen des Landesamtes für Denkmalpflege und Archäologie Sachsen-Anhalt - Landesmuseum für Vorgeschichte, 62: 289-303.

Busschers, F.S., Van Balen, R. T., Cohen, K. M., Kasse, C., Weerts, H. J. T., Wallinga, J. \& BunniK, F. P. M. (2008): Response of the Rhine-Meuse fluvial system to Saalian ice-sheet dynamics. - Boreas, 37: 377-398.

Clessin, S. (1877): Deutsche Excursions-Mollusken-Fauna. - 581 S.; Nürnberg (1876-1877).

Degering, D. \& KRBETscheK, M. R. (2007a): Dating of interglacial deposits by luminescence methods. - In: Sirocko, F., Claussen, M., Sánchez Goni, M. F. \& Litt, T. (eds.), The Climate of Past Interglacials, Developments in Quaternary Science, 7: 157-172; Elsevier (Amsterdam).

Degering, D. \& KRBETSCheK, M. R. (2007b): Lumineszenzdatierungen an limnischen Sedimenten von Klinge/Niederlausitz. - Natur und Landschaft in der Niederlausitz, 24: 120-128.

DIN 18128 (Ausgabe 2002-12): Baugrund - Untersuchung von Bodenproben - Bestimmung des Glühverlustes.

DIN 18129 (Ausgabe 1996-11): Baugrund - Untersuchung von Bodenproben - Kalkgehaltsbestimmung.

DIN ISO 10694 (Ausgabe 1996-08): Bodenbeschaffenheit - Bestimmung von organischem Kohlenstoff und Gesamtkohlenstoff nach trockener Verbrennung (Elementaranalyse).

Dütsch, C. ¿ KrbetscheK, M. R. (1997): New methods for a better internal K-40 dose rate determination. - Radiation Measurements, 27: 377-381.

Ehlers, J., Eissmann, L., Lippstreu, L., Stephan, H.-J. \& WANSA, S. (2004): Pleistocene Glaciations of North Germany. - In: Ehlers, J. \& Gibbard, P. (eds.), Quaternary Glaciations - Extent and Chronology, Part I: Europe, Developments in Quaternary Science, 2a: 135-146; Amsterdam.

EIsSMANN, L. (1975): Das Quartär der Leipziger Tieflandsbucht und angrenzender Gebiete um Saale und Elbe. - Schriftenreihe für geologische Wissenschaften, 2: 1-263.
EissmanN, L. (1981): Periglaziäre Prozesse und Permafroststrukturen aus sechs Kaltzeiten des Quartärs. - Altenburger naturwiss. Forschungen, 1: $1-171$.

EIsSMANN, L. (1987): Lagerungsstörungen im Lockergebirge. Exogene und endogene Tektonik im Lockergebirge des nördlichen Mitteleuropa. Geophysik u. Geologie, III: 7-77.

ERD, K. (1973): Pollenanalytische Gliederung des Pleistozäns der Deutschen Demokratischen Republik. - Zeitschrift für geologische Wissenschaften, 1: $1087-1103$.

ERD, K. (1979): Bericht über die pollenanalytische Untersuchung eines Profils aus der Baugrube Eisenbahneinschnitt Lug 1966. - unveröff. Bericht, ZGI Berlin vom 29.03.1979 (Archiv-Nr. 2018826)

ERD, K. (1987): Die Uecker-Warmzeit von Röpersdorf bei Prenzlau als neuer Interglazialtyp im Saale-Komplex der DDR. - Zeitschrift für geologische Wissenschaften, 15: 297-313.

ERD, K. \& Strahl, J. (2008): Abbruchbericht zur pollenanalytischen Bearbeitung der Bohrung Kt 8778Z/88 Gräbendorf-Reddern (Brk Gäd 8778Z/88), Land Brandenburg. - unveröff. Bericht, LBGR Kleinmachnow vom 11.02.2008

ErfuRt, G. \& KrbetscheK, M. R. (2003): IRSAR - A single-aliquot regenerative-dose dating protocol applied to the infrared radiofluorescence (IR-RF) of coarse-grain K feldspar. - Ancient TL, 21: 25-43.

FunrmanN, R. (2006): Die Ostrakodenfauna des Interglazialbeckens Neumark Nord 2 und ihre Aussage zur stratigraphischen Stellung des Interglazials von Neumark Nord. - Veröffentlichungen des Landesmuseums für Vorgeschichte in Halle, 43: 167-175

GeyH, M. A. (2008): The handling of numerical ages and their random uncertainties. - Eiszeitalter und Gegenwart (Quaternary Science Journal), 57: 239-252

GolDFuss, O. (1900): Die Binnenmollusken Mittel-Deutschlands mit besonderer Berücksichtigung der Thüringer Lande, der Provinz Sachsen, des Harzes, Braunschweigs und der angrenzenden Landesteile. - 320 S.; Leipzig.

GöTtinG, K.-J. (2008): Meeres-Gehäuseschnecken Deutschlands. - Die Tierwelt Deutschlands, 80. Teil, 180 S. (ConchBooks).

GrichuK, V. P. (1940): Method of treatment of the sediments poor in organic remains for the pollen analysis. - Problemy fizicheskoi geografii, 8: 53-58.

GRÜN, R. (1992): AGE-Software for dose rate and age calculation in luminescence and ESR dating (delivered by Risoe Nat. Laboratories, Denmark)

HeINRICH, W.-D. (2001): Kleinsäugerreste aus interglazialen Ablagerungen von Neumark Nord, Mitteldeutschland. - Praehistoria Thuringica, 6/7: $132-138$.

HeINRICH, W.-D. (2010): Kleinsäugerreste aus den pleistozänen Beckenablagerungen von Neumark-Nord 1. - Veröffentlichungen des Landesamtes für Denkmalpflege und Archäologie Sachsen-Anhalt - Landesmuseum für Vorgeschichte, 62: 327-337.

Hermsdorf, N. \& Strahl, J. (2006): Zum Problem der so genannten UeckerWarmzeit (Intra-Saale) - Untersuchungen an neuen Bohrkernen aus dem Raum Prenzlau. - Brandenburgische geowissenschaftliche Beiträge, $13(1 / 2): 49-61$.

Hinze, C., Jerz, H., Menke, B. \& Staude, H. (1989): Geogenetische Definitionen quartärer Lockergesteine für die Geologische Karte 1:25000 (GK 25). - Geologisches Jahrbuch, A 112: 243 S.; Hannover.

Huntley, D. J. \& BARIL, M. R. (1997): The K content of the K-feldspars being measured in optical dating or thermoluminescence dating. - Ancient TL 15: 11-13.

Junge, F. W. (1998): Die Bändertone Mitteldeutschlands und angrenzender Gebiete. - Altenburger naturwiss. Forschungen, 9: 1-210.

KAHLKE, R.-D. (2002): The Quaternary large mammal faunas of Thuringia (Central Germany). - In: Meyrik, R. A. \& Schreve D. C. (Hrsg.): The Quaternary of Central Germany. Field Guide. Quaternary Research Association: 59-78; London.

KARELIN, P. (1997): Untersuchungen zur Datierungsanwendung des $325^{\circ} \mathrm{C}$ TL-Peaks in Quarzen aus dem nordischen Vereisungsgebiet Deutschlands. - Dissertation., Univ. Heidelberg: $126 \mathrm{~S}$.

KAUFMAN, D. S. \& MANLEY, W. F. (1998): A new procedure for determining DL amino acid ratios in fossils using reverse phase liquid chromatography. - Quaternary Science Reviews, 17(11): 987-1000.

KrbetscheK, M. R., Degering, D. \& Alexowsky, W. (2008): Infrarot-Radiofluoreszenz-Alter (IR-RF) unter-saalezeitlicher Sedimente Mittel- und Ostdeutschlands. [Infrared radiofluorescence ages (IR-RF) of Lower Saalian sediments from Central and Eastern Germany.] - Zeitschrift der Deutschen Gesellschaft für Geowissenschaften, 159 (1): 133-140. 
Krbetschek, M. R., Dietrich, A. \& Trautmann, T. (1998): Datierung pleistozäner Sedimente mittels Optisch Stimulierter Lumineszenz. Forsch.-Bericht (Teile 1 und 2), Sächsisches Landesamt für Umwelt u. Geologie: 37 S. / 15 S.

KrbetscheK, M. R. \& Eissmann, L. (2008) Geochronologische Daten aus der Typregion der Elster- und Saale-Inlandvergletscherungen. - Abhandlungen der Geologischen Bundesanstalt (Wien), 62: 169-171.

KrbetscheK, M. R., Rieser, U., Zöller, L. \& Heinicke, J. (1994): Radioactive disequilibria in palaeodosimetric dating of sediments. - Radiation Measurements, 23: 485-489.

Krbetscheк, M. R. \& Stolz, W. (1994): Lumineszenz-Datierung an pleistozänen Sedimenten aus Tagebauen des Mitteldeutschen und Lausitzer Braunkohlenreviers. - Altenburger naturwiss. Forschungen, 7: 289-295

Krbetscheк, M. R. \& Stolz, W. (1997): Lumineszenz-Datierungen am jungpleistozänen Becken von Gröbern - neue Ergebnisse. - Leipziger Geowissenschaften, 5: 129-133.

Kremenetski, K. V. (2010): Neumark-Nord: Basin N.-N. 2 - Results of the pollen analysis. - Veröffentlichungen des Landesamtes für Denkmalpflege und Archäologie Sachsen-Anhalt - Landesmuseum für Vorgeschichte, 62: 273-287.

KüHNER, R. (2003): Ausbildung und Gliederung des saalezeitlichen Sedimentkomplexes im Bereich der Hornoer Hochfläche. - Brandenburgische Geowissenschaftliche Beiträge, 10 (1/2): 111-121.

Kulig, G. (2005) Erstellung einer Auswertesoftware zur Altersbestimmung mittels Lumineszenzverfahren. - Fakultät für Mathematik und Informatik, TU Freiberg (2005).

LANG, G. (1994): Quartäre Vegetationsgeschichte Europas. - 462 S., Jena (Gustav Fischer)

LAURAT, T. \& BRÜHL, E. (2006): Zum Stand der archäologischen Untersuchungen im Tagebau Neumark Nord, Ldkr. Merseburg-Querfurt (Sachsen-Anhalt). Vorbericht zu den Ausgrabungen 2003-2005. - Jahresschriften für mitteldeutsche Vorgeschichte, 90: 9-69.

Laurat, T., BrüHL, E., Hesse N. \& Jurkenas, D. (2007): Die Schichtenfolge des Seebeckens Neumark-Nord 2. Beschreibung der im Grabungsarea aufgeschlossenen Schichten des Drenthe bis Holozän und Bericht zum Forschungsstand (Stand September 2007). - unveröff. Bericht, Landesamt für Denkmalpflege und Archäologie Sachsen-Anhalt.

Laurat, T., Brühl, E. \& JurkenAs, D. (2004): Frühe Menschen an der Geisel - Die Ausgrabungen im Tagebau Neumark-Nord (Ldkr. MerseburgQuerfurt). - unveröff. Bericht, Landesamt für Denkmalpflege und Archäologie Sachsen-Anhalt.

LAurAt, T., BrüHL, E. \& JurkenAs, D. (2006): Halt 2-6: Quartärgeologie und Archäologie im Tagebau Neumark-Nord. - 73. Tagung der AG Norddeutscher Geologen, Juni 2006, Halle, Tagungsband und Exkursionsführer: 81-91; Halle.

LITT, T. (1990): Pollenanalytische Untersuchungen zur Vegetations- und Klimaentwicklung während des Jungpleistozäns in den Becken von Gröbern und Grabschütz. - Altenburger naturwiss. Forschungen, 5: 92-105.

LitT, T. (1994a): Paläoökologie, Paläobotanik und Stratigraphie des Jungquartärs im nordmitteleuropäischen Tiefland. - Dissertationes Botanicae, 227: 185 S.; Berlin, Stuttgart.

LITT, T. (1994b): Zur stratigraphischen Einstufung des Interglazials von Neumark-Nord aufgrund neuer pollenanalytischer Befunde. - Altenburger naturwiss. Forschungen, 7: 328-333.

Litt, T., Behre, K.-E., Meyer, K.-D., Stephan, H.-J. \& Wansa, S. (2007): Stratigraphische Begriffe für das Quartär des norddeutschen Vereisungsgebietes. - Eiszeitalter und Gegenwart (Quaternary Science Journal), 56 (1/2): 7-65.

Litt, T. \& Gibbard, P. (2008): A proposed Global Stratotype Section and Point (GSSP) for the base of the Upper (Late) Pleistocene Subseries (Quaternary System/Period). - Episodes, 31 (2) Special Issue on the Quaternary Period/System: 260-263.

Litt, T. \& Wansa, S. (2008): QuARtÄr. - In: Bachmann, G., Ehling, B.-C., EICHNER, R. \& SchwAB, M. (Hrsg.), Geologie von Sachsen-Anhalt, 293325; Stuttgart (Schweizerbart).

Machalett, B. (2010): Past Atmospheric Circulation Patterns and Aeolian Dust Dynamics Recorded in Eurasian Loess: Utilizing high-resolution particle size analysis and amino acid geochronology. - Dissertation, Humboldt-Universität zu Berlin, Geographisches Institut: 120 S.; Berlin (Mensch \& Buch Verlag).

Machalett, B., Oches, E. A., Frechen, M., Zöller, L., Hambach, U., MaVlyanova, N. G., Markovic, S. B. \& Endlicher, W. (2008): Aeolian dust dynamics in central Asia during the Pleistocene: Driven by the longterm migration, seasonality, and permanency of the Asiatic polar front. - Geochem. Geophys. Geosyst., 9, Q08Q09.
MAI, D. H. (1990): Die Flora des Interglazials von Neumark-Nord, Kr. Merseburg. - Veröffentlichungen des Landesmuseums für Vorgeschichte in Halle, 43: 159-160.

MAI, H. D. (1992): Über einige Steppen- und Salzpflanzen in sächsisch-thüringischen Interglazialen und ihre vegetationsgeschichtliche Bedeutung. - Gleditschia, 20: 57-85.

ManiA, D. (1990): Stratigraphie, Ökologie und mittelpaläolithische Jagdbefunde des Interglazials von Neumark-Nord (Geiseltal). - Veröffentlichungen des Landesmuseums für Vorgeschichte in Halle, 43: 9-130.

MAniA, D. (1992): Neumark-Nord - ein fossilreiches Interglazial im Geiseltal. - Cranium, 9 (2): 53-76.

MANIA, D. (1994): Das Interglazialvorkommen von Neumark-Nord. - Altenburger naturwiss. Forschungen, 7: 324-327.

ManiA, D. (2000): Zur Paläontologie des Interglazials von Neumark-Nord im Geiseltal. - Praehistoria Thuringica, 4: 67-94.

MANIA, D. (2004): Neumark-Nord - Geologie eines Interglazials. - Praehistoria Thuringica, 10: 26-42.

ManiA, D. (2010a): Mittelpaläolithische Funde (Frühglazial, Weichsel-Kaltzeit) aus dem Geiseltal in Verbindung mit der Fundgemeinschaft des Elbe-Saale-Gebietes. - Veröffentlichungen des Landesamtes für Denkmalpflege und Archäologie Sachsen-Anhalt - Landesmuseum für Vorgeschichte, 62: 211-266.

ManiA, D. (2010b): Zur Geologie, Paläontologie und Archäologie der Körbisdorfer Schotter (Geiseltal) im mittelpleistozänen Holstein-Komplex des Elbe-Saale-Gebietes. - Veröffentlichungen des Landesamtes für Denkmalpflege und Archäologie Sachsen-Anhalt - Landesmuseum für Vorgeschichte, 62: 81-119.

Mania, D. unter Mitarb. von Altermann, M., Böhme, G., Böttger, T., Brühl, E., Döhle, H.-J., ERd, K., Fischer, K., Fuhrmann, R., Heinrich, W.-D., Grube, R., Karelin, P. G., Koller, J., Kremenetski, K. V., LaUrat, T., Van der Made, J., Mai, D. H. Mania, U., Musil, R., PfeifferDeml, T., Pietrzeniuk, E., Schüler, T., Seifert-Eulen, M. \& Thomae, M. (2010): Quartärforschung im Tagebau Neumark-Nord, Geiseltal (Sachsen-Anhalt und ihre bisherigen Ergebnisse. - Veröffentlichungen des Landesamtes für Denkmalpflege und Archäologie Sachsen-Anhalt - Landesmuseum für Vorgeschichte, 62: 11-69.

Mania, D., Altermann, M., Böhme, G., Erd, K., Fischer, K., Heinrich, W.-D., Krementzki, C., V. D. Made, J., Mai, D. H., Musil, R., PietrzeNIUK, E., SCHÜler, T., VlceK, I. ש STEINER, W. (2003): Die Travertine in Thüringen und im Harzvorland. - Hallesches Jahrbuch für Geowissenschaften, Reihe B, Beiheft 17: 1-82.

ManiA, D. \& MAI, D. H. (1969): Warmzeitliche Mollusken- und Pflanzenreste aus dem Mittelpleistozän des Geiseltals (südlich von Halle). - Geologie, 18: 674-690.

Mania, D., Mania, U. \& Thomae, M. (2003): Im Wildparadies des Geiseltales vor 200000 Jahren. - Interessen- und Förderverein „Geiseltalsee“ e. V., 41 S.; Mücheln.

Mania, D. \& Thomae, M. (1987): Neumark-Nord - Fundstätte eines interglazialen Lebensraumes mit anthropogenen Besiedlungsspuren. Ein Vorbericht. - Technische Kurzinformation, 23 (H. 43): 32-51; Braunkohlenwerk Geiseltal.

Mania, D. \& Thomae, M. unter Mitarb. von Altermann, M., Heinrich W.-D., VAn der Made, J., Mai, D. H. \& SeIfERT-Eulen, M. (2008): Zur stratigraphischen Gliederung der Saalezeit im Saalegebiet und Harzvorland. - Praehistoria Thuringica, Sonderheft: 1-44.

McCreA, J. M. (1950): On the Isotopic Chemistry of the carbonates and a paleotemperature scale. - Journal of Chemical Physics, 18: 849-857.

Meng, S., Nuglisch, K., Scharf, B. \& Becker, A. (2004): Neue Daten zur holozänen Faunengeschichte (Foraminifera, Ostracoda, Mollusca: Gastropoda) des Süßen Sees bei Halle/Saale (Sachsen-Anhalt) mit einigen Bemerkungen zur Seegenese. - Hallesches Jahrbuch für Geowissenschaften, B 26: 119-134.

Menke, B. \& Tynni, R. (1984): Das Eem-Interglazial und das FrühweichselGlazial von Rederstall/Dithmarschen und ihre Bedeutung für die mitteleuropäische Jungpleistozän-Gliederung. - Geologisches Jahrbuch, A 76: 3-120.

Merkt, J., LÜttig, G. \& Schneekloth, H. (1971): Vorschlag zur Gliederung und Definition limnischer Sedimente. - Geologisches Jahrbuch, 89: 607-623.

MEusEL, H. (1970): Verbreitungsgrenzen südlicher Florenelemente in Mitteldeutschland. - Feddes Repertorium, 81: 289-309.

Meyer, K.-D. (2005): Zur Stratigraphie des Saale-Glazials in Niedersachsen und zu Korrelationsversuchen mit Nachbargebieten. - Eiszeitalter und Gegenwart, 55: 25-42. 
Moore, D. M. ¿ ReYNoLDS, R. C., JR. (1997): X-Ray Diffraction and the Identification and Analysis of Clay Minerals. - 378 S.; Oxford.

MüLLER, H. (1974): Pollenanalytische Untersuchungen und Jahresschichtenzählungen an der eemzeitlichen Kieselgur von Bispingen/Luhe. - Geologisches Jahrbuch, A 21:149-169; Hannover.

MurRAY, A. S. ¿ WintLE, A. G. (2000): Luminescence dating of quartz using an improved single-aliquot regenerative-dose protocol. - Radiation Measurements, 33: 57-73.

Oches, E. A. \& McCoy, W. D. (1995): Amino acid geochronology applied to the correlation and dating of central European loess deposits. - Quaternary Science Reviews, 14 (7-8): 767-782.

Oches, E. A. \& McCoy, W. D. (2001): Historical developments and recent advances in amino acid geochronology applied to loess research: examples from North America, Europe, and China. - Earth Science Reviews, 54 (1-3): 173-192.

Oches, E. A., MCCoy, W. D. \& CLARK, P. U. (1996): Amino acid estimates of latitudinal temperature gradients and geochronology of loess deposition during the last glaciation, Mississippi Valley, United States. - Geological Society of America Bulletin, 108: 892-903.

Oches, E. A., McCoy, W. D. \& GenIESER, D. (2000): Aminostratigraphic correlation of loess - paleosol sequences across Europe. - In: Goodfriend, G., Collins, M., Fogel, M., Macko, S. \& Wehmiller, J. F.: Perspectives in Amino Acid and Protein Geochemistry, 331-348; Oxford, New York (University Press)

Preusser, F., Degering, D., Fuchs, M., Hilgers, A., Kadereit, A., Klasen, N., Krbetschek, M., Richter, D. \& Spencer, J. Q. G. (2008): Luminescence dating: basics, methods and applications. - Eiszeitalter und Gegenwart (Quaternary Science Journal): 57 (1/2): 95-149.

RAPPSILBER, I. (2004a): Neumark-Nord - Auswertung von Georadarmessungen zur Untersuchung des geologischen Untergrundes. - unveröff. Bericht, Archiv LAGB Sachsen-Anhalt.

RAPPSILBER, I. (2004b): Neumark-Nord - Auswertung geoelektrischer Messungen zur Untersuchung des geologischen Untergrundes. - unveröff. Bericht, Archiv LAGB Sachsen-Anhalt.

REYNoLDS, R. C., JR. (1984): Interstratified clay minerals. - In: Brindley, G. W. \& G. Brown (eds.): Crystal structures of clay minerals and their Xray identification. - Mineralogical Soc., 249-303; London.

Ruske, R. (1961): Gliederung des Pleistozäns im Geiseltal und in seiner Umgebung. - Geologie, 10: 152-168.

Schulz, W. (1962): Gliederung des Pleistozäns in der Umgebung von Halle (Saale). - Geologie, Beih. 36: 1-69.

SeIfERT, M. (1990): Ein Interglazial von Neumark-Nord (Geiseltal) im Vergleich mit anderen Interglazialvorkommen in der DDR. - Veröffentlichungen des Landesmuseums für Vorgeschichte in Halle, 43: 149-158.

SeIfERT-EulEN, M. (2010): Vegetationsgeschichte des Interglazials von Neumark-Nord (Becken N.-N. 1). - Veröffentlichungen des Landesamtes für Denkmalpflege und Archäologie Sachsen-Anhalt - Landesmuseum für Vorgeschichte, 62: 267-272.

StRAhL, J. (2004): Bericht über die pollenanalytische Untersuchung der Kernbohrungen Ww 3/03 (KB Tschernitz SN-3/2003) und Ww 5/03 (KB Tschernitz SN-5/2003), Land Brandenburg. - unveröff. Bericht, LBGR Kleinmachnow vom 15.10.2004.
StRAhL, J. (2007): Pollenanalytische Revisionsbearbeitung der Bohrungen Nedlitz 1/87, 1A/87 und 3/88 (Land Sachsen-Anhalt). - unveröff. Bericht, LBGR Kleinmachnow vom 06.02.2007.

StRAhL, J. ש HeRMSDORF, N. (2008): Karte der Eem-Vorkommen des Landes Brandenburg. - Brandenburgische geowissenschaftliche Beiträge, 15 (1): 23-55.

THомав, M. (1986): Zur Genese pleistozäner Lagerungsstörungen im oberen Deckgebirge des Geiseltales. - Technische Kurzinformation, 22 (H. 41): 20-26; Braunkohlenwerk Geiseltal.

THомAe, M. (1990): Geologischer Bau und Lagerungsverhältnisse des Quartärprofils von Neumark-Nord. - Veröffentlichungen des Landesmuseums für Vorgeschichte in Halle, 43: 131-143.

Thомав, M. (2003): Mollisoldiapirismus - Ursache für die Erhaltung der Fundstätte Neumark-Nord (Geiseltal). - In: Justus, A., Fiedler, L., Burdukiewicz, J. M., Heinrich, W.-D. \& Brühl, E. (Hrsg.): Erkenntnisjäger Kultur und Umwelt des frühen Menschen. Festschrift für Dietrich Mania, Veröffentlichungen des Landesamtes für Archäologie Sachsen-Anhalt, 57 (II): 509-535.

THOMAE, M. ¿ RAPPsiLber, I. (2010): Beitrag zur Klärung der Lagerungsverhältnisse des Quartärs im Tagebau Neumark-Nord. - Veröffentlichungen des Landesamtes für Denkmalpflege und Archäologie Sachsen-Anhalt - Landesmuseum für Vorgeschichte, 62: 211-266.

ToEPfer, V. (1957): Die Mammutfunde von Pfännerhall im Geiseltal. - Veröffentlichungen des Landesmuseums für Vorgeschichte in Halle, 16: $1-58$.

Trautmann, T., KrbetscheK, M. R, Dietrich, A. \& Stolz, W. (1999): Feldspar radioluminescence: A new dating method and its physical background. - Journal of Luminescence, 85: 45-58.

VAN DER MADE, J. (2003): Megaloceros giganteus from the Middle Pleistocene of Neumark Nord. - In: Justus, A., Fiedler, L., Burdukiewicz, J. M., Heinrich, W.-D. \& Brühl, E. (Hrsg.): Erkenntnisjäger: Kultur und Umwelt des frühen Menschen. Festschrift für Dietrich Mania, Veröffentlichungen des Landesamtes für Archäologie Sachsen-Anhalt - Landesmuseum für Vorgeschichte, 57 (II): 373-378.

VAn der MAde, J. (2010): The rhinos from the Middle Pleistocene of Neumark Nord (Saxony-Anhalt). - Veröffentlichungen des Landesamtes für Denkmalpflege und Archäologie Sachsen-Anhalt - Landesmuseum für Vorgeschichte, 62: 433-527.

WANSA, S (2008): Zeitz-Glaziär-Formation. - In: LithoLex [Online-Datenbank]; Hannover (BGR).

WANSA, S. ¿ RADZINSKI, K.-H. (2004): Erläuterungen zur Geologischen Karte von Sachsen-Anhalt 1:25 000, Blatt 4636 Mücheln (Geiseltal). - 143 S.; Halle

Wehmiller, J. F. \& Miller, G. H. (2000), Aminostratigraphic Dating Methods in Quaternary Geology: Methods and Applications. - AGU reference shelf (ed.): 187-222; AGU, Washington, D. C.

WinTLE, A. G. (1997): Luminescence dating: Laboratory procedures and protocols. - Radiation Measurements, 27: 769-817.

WINTLE, A. G. (2008): Luminescence dating: where it has been and where it is going. - Boreas, 37 : 471-482.

ZöLler, L. (2010). New approaches to European loess: a stratigraphic and methodical review of the past decade. - Central European Journal of Geosciences, 2 (1): 19-31. 